Florida International University FIU Digital Commons

\title{
Design, Fabrication, and Evaluation of On-chip Micro-supercapacitors
}

Majid Beidaghi

Florida International University, mbeid001@fiu.edu

DOI: $10.25148 /$ etd.FI12071111

Follow this and additional works at: https://digitalcommons.fiu.edu/etd

\section{Recommended Citation}

Beidaghi, Majid, "Design, Fabrication, and Evaluation of On-chip Micro-supercapacitors" (2012). FIU Electronic Theses and Dissertations. 660.

https://digitalcommons.fiu.edu/etd/660 


\section{FLORIDA INTERNATIONAL UNIVERSITY}

Miami, Florida

\section{DESIGN, FABRICATION, AND EVALUATION OF ON-CHIP MICRO- SUPERCAPACITORS}

A dissertation submitted in partial fulfillment of the requirements for the degree of DOCTOR OF PHILOSOPHY

in

MATERIALS SCIENCE AND ENGINEERING

by

Majid Beidaghi

2012 


\section{To: Dean Amir Mirmiran}

College of Engineering and Computing

This dissertation, written by Majid Beidaghi, and entitled Design, Fabrication, and Evaluation of On-chip Micro-supercapacitors, having been approved in respect to style and intellectual content, is referred to you for judgment.

We have read this dissertation and recommend that it be approved.

$\begin{array}{r}\text { W. Kinzy Jones } \\ \hline \text { Norman D.H. Munroe }\end{array}$

Wenzhi Li

Chunlei Wang, Major Professor

Date of Defense: May 31, 2012

The Dissertation of Majid Beidaghi is approved.

$\begin{array}{r}\text { Dean Amir Mirmiran } \\ \text { College of Engineering and Computing } \\ \hline \begin{array}{r}\text { Dean Lakshmi N. Reddi } \\ \text { University Graduate School }\end{array}\end{array}$

Florida International University, 2012 
(C) Copyright 2012 by Majid Beidaghi

All rights reserved. 


\section{DEDICATION}

I dedicate this dissertation to my loving parents, and lovely fiancé. Without their love, support, and patience, it would have been impossible to complete this research. 


\section{ACKNOWLEDGMENTS}

I am deeply grateful to my advisor, Dr. Chunlei Wang, for her guidance, vision and support throughout the course of my $\mathrm{PhD}$ research. I express my special thanks to her for reviewing my research works and providing constructive comments to improve the quality of my research. I also cordially appreciate her inspiration and invaluable encouragement for my future career development.

My gratitude is extended to Dr. W. Kinzy Jones, Dr. Norman D.H. Munroe and Dr. Wenzhi Li for their willingness to serve on my dissertation committee and for their invaluable support and encouragement.

I wish to thank Mr. Neal Ricks for his support for my experiments throughout my research period. I have learnt from him and without his support I would not have been able to finish my dissertation in a timely manner.

I sincerely thank my present and past research group members: Dr. Wei Chen, Dr. Xifei Li, Dr. Yan Yu, Varun Penmatsa, Abirami Dhanabalan, Chunhui Chen, Yin Song, Tae Kwon Kim, Kevin Bechtold, Richa Agarwal, and Yong Hao.

I acknowledge the University Graduate School at FIU for supporting me through

Dissertation Evidence Acquisition (DEA) and Dissertation Year Fellowship (DYF) awards.

My heartfelt thanks to my dear friends Pedram, Nasim, Babak, Bita, Rambod, Yalda and Shaahin for always being there when I needed them during the past years.

I also sincerely thank Dr. Pirouz Daftarian, Mrs. Daftarian, Maryam and Ali Daftarian for their support and encouragement. 
I am grateful to my parents for their endless support and love. They have patiently faced my absence during the past four and half years.

Finally, I would like to thank my lovely fiancé, Zahra, for her understanding and support. I spent many a time that should have been spent with her working on my research and I hope that I can make up for this in the future. I also wish to thank her for always helping me edit my writings. 


\title{
ABSTRACT OF THE DISSERTATION \\ DESIGN, FABRICATION, AND EVALUATION OF ON-CHIP MICRO- SUPERCAPACITORS
}

\author{
by
}

Majid Beidaghi

Florida International University, 2012

Miami, Florida

\section{Professor Chunlei Wang, Major Professor}

Due to the increasing demand for high power and reliable miniaturized energy storage devices, the development of micro-supercapacitors or electrochemical microcapacitors have attracted much attention in recent years. This dissertation investigates several strategies to develop on-chip micro-supercapacitors with high power and energy density.

Micro-supercapacitors based on interdigitated carbon micro-electrode arrays are fabricated through carbon microelectromechanical systems (C-MEMS) technique which is based on carbonization of patterned photoresist. To improve the capacitive behavior, electrochemical activation is performed on carbon micro-electrode arrays. The developed micro-supercapacitors show specific capacitances as high as $75 \mathrm{mFcm}^{-2}$ at a scan rate of 5 $\mathrm{mVs}^{-1}$ after electrochemical activation for 30 minutes. The capacitance loss is less than $13 \%$ after 1000 cyclic voltammetry (CV) cycles. These results indicate that electrochemically activated C-MEMS micro-electrode arrays are promising candidates for on-chip electrochemical micro-capacitor applications. 
The energy density of micro-supercapacitors was further improved by conformal coating of polypyrrole (PPy) on C-MEMS structures. In these types of micro-devices the three dimensional (3D) carbon microstructures serve as current collectors for high energy density PPy electrodes. The electrochemical characterizations of these microsupercapacitors show that they can deliver a specific capacitance of about $162.07 \mathrm{mFcm}^{-2}$ and a specific power of $1.62 \mathrm{mWcm}^{-2}$ at a $20 \mathrm{mVs}^{-1}$ scan rate.

Addressing the need for high power micro-supercapacitors, the application of graphene as electrode materials for micro-supercapacitor was also investigated. The present study suggests a novel method to fabricate graphene-based micro-supercapacitors with thin film or in-plane interdigital electrodes. The fabricated micro-supercapacitors show exceptional frequency response and power handling performance and could effectively charge and discharge at rates as high as $50 \mathrm{Vs}^{-1}$. CV measurements show that the specific capacitance of the micro-supercapacitor based on reduced graphene oxide and carbon nanotube composites is $6.1 \mathrm{mFcm}^{-2}$ at scan rate of $0.01 \mathrm{Vs}^{-1}$. At a very high scan rate of $50 \mathrm{Vs}^{-1}$, a specific capacitance of $2.8 \mathrm{mFcm}^{-2}$ (stack capacitance of $3.1 \mathrm{Fcm}^{-3}$ ) is recorded. This unprecedented performance can potentially broaden the future applications of micro-supercapacitors. 


\section{TABLE OF CONTENTS}

CHAPTER

PAGE

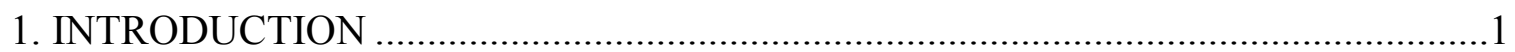

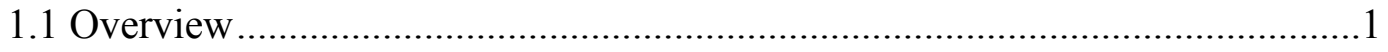

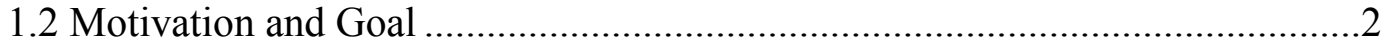

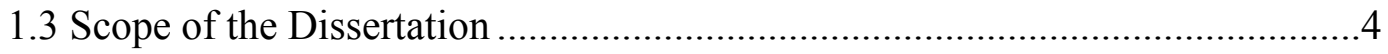

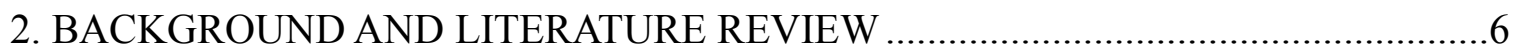

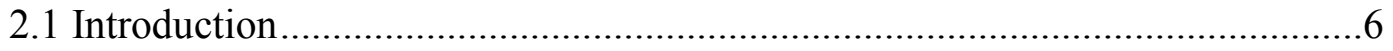

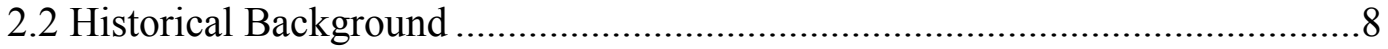

2.3 Electrochemical Properties of Supercapacitor ...................................................

2.4 Charge Storage Mechanisms Supercapacitors ................................................11

2.5 Electrode Materials for Supercapacitors …………….......................................11

2.6 Miniaturized Power Sources ........................................................................

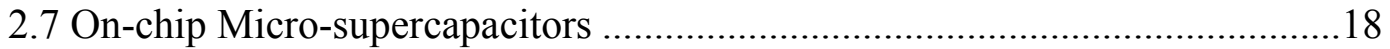

2.7.1 Architecture of Micro-supercapacitors ............................................18

2.7.2 Thin Film Micro-supercapacitors ...................................................2.

2.7.3 Micro-supercapacitor with In-plane Interdigital Electrodes ..............22

2.7.4 Micro-supercapacitors with 3D Electrodes.......................................25

2.8 Carbon-Microelectromechanical Systems (C-MEMS) .....................................26

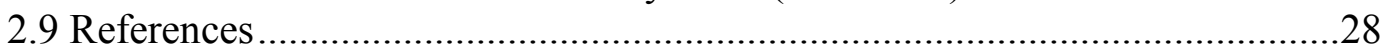

3. ELECTROCHEMICALLY ACTIVATED CARBON MICRO-ELECTRODE ARRAYS FOR ON-CHIP MICRO-SUPERCAPACITOR APPLICATION …………......................33

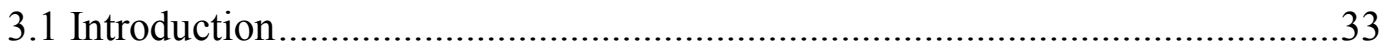

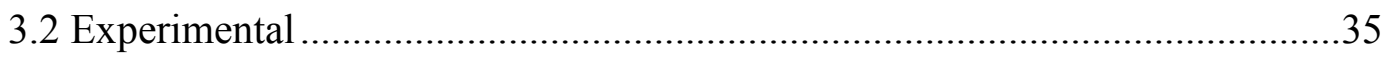

3.2.1 Preparation of C-MEMS Electrodes ..................................................35

3.2.2 Electrochemical Activation ............................................................36

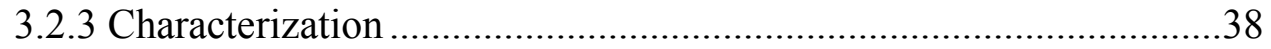

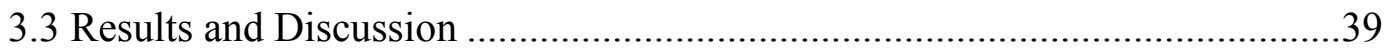

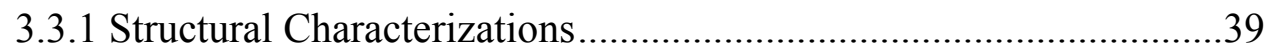

3.3.2 Electrochemical Tests ……………………………......................... 40

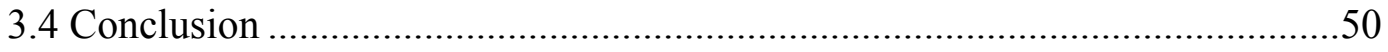

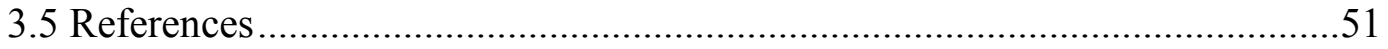

4. MICRO-SUPERCAPACITORS BASED ON THREE DIMENSIONAL

INTERDIGITAL POLYPYRROLE/C-MEMS ELECTRODES ………..........................53

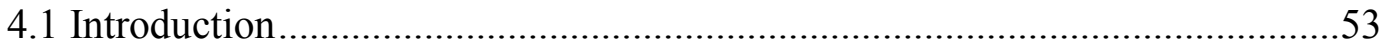

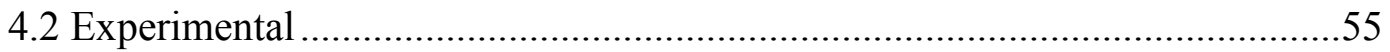

4.2.1 Design of the Electrodes ..................................................................5

4.2.2 Fabrication of C-MEMS Structures ……………………………........56

4.2.3 Electropolymerization of PPy Films ................................................57

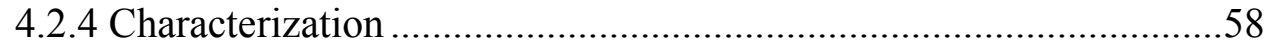




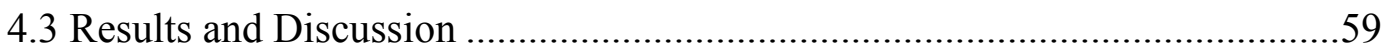

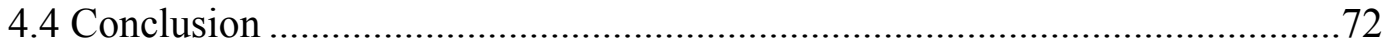

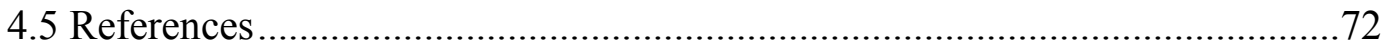

5. ELECTROSTATIC SPRAY DEPOSITION OF GRAPHENE NANOPLATELETS FOR HIGH POWER THIN FILM SUPERCAPACITOR ELECTRODES ....................74

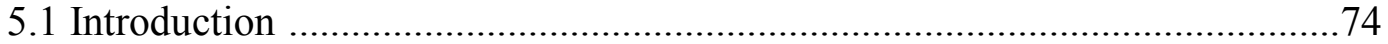

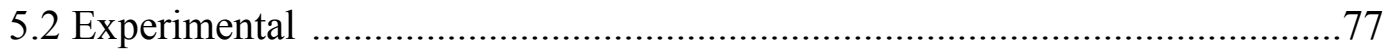

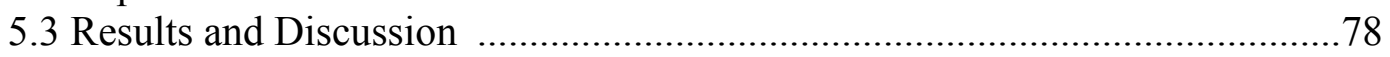

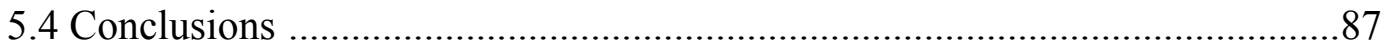

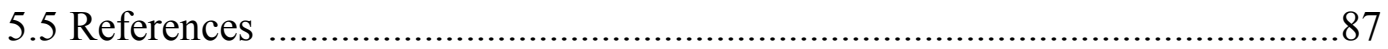

6. MICRO-SUPERCAPACITORS BASED ON INTERDIGITAL ELECTRODES OF REDUCED GRAPHENE OXIDE AND CNT COMPOSITES .....................................90

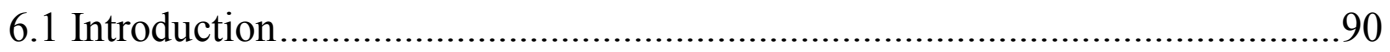

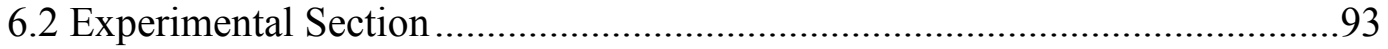

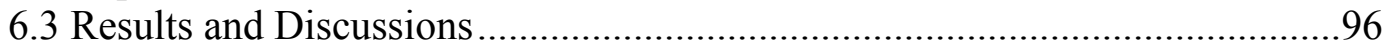

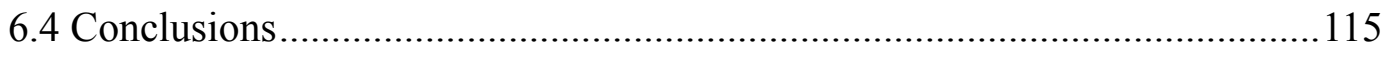

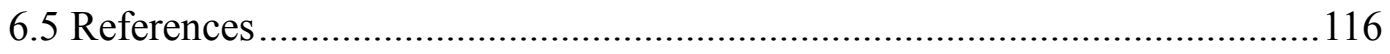

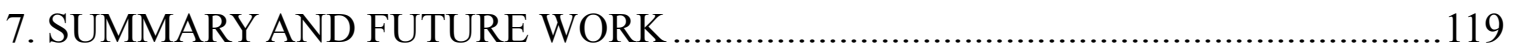

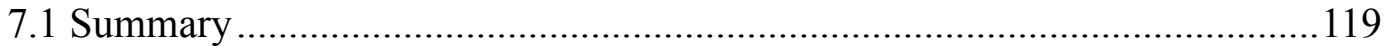

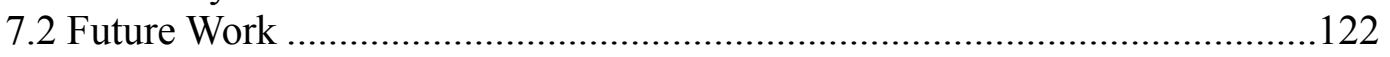

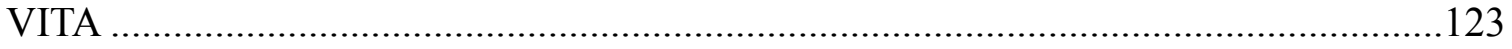




\section{LIST OF FIGURES}

FIGURE

PAGE

Figure 1.1 Flow chart of the research plan. .5

Figure 2.1 Ragone plot of specific power versus specific energy for various electrical energy storage and conversion devices.

Figure 2.2 representation of charge storage mechanisms in (a) a conventional capacitor, (b) EDLC, (c) pseudo-capacitor, and (d) a battery. 10

Figure 2.3 Specific capacitance reported for various supercapacitor electrode materials. 16

Figure 2.3 Three different device architectures for on-chip micro-supercapacitors. (a) 2D planar, (b) in-plane interdigital, and (c) two examples of possible 3D designs.................20

Figure 2.4 Process flow for fabrication of C-MEMS microstructures.............................27

Figure 2.5 Typical SEM micrographs of SU-8 structures before pyrolysis (a, b), and

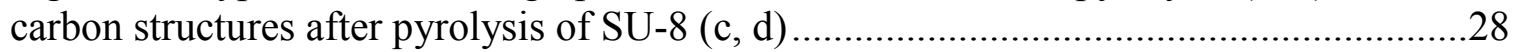

Figure 3.1 (a) Schematic of a typical process flow for fabricating of C-MEMS electrodes. (b) Schematic $3 \mathrm{D}$ view of a sample after carbonization....

Figure 3.2 SEM images of C-MEMS electrodes. (a) SU-8 structure (b) carbonized structure. The insets show higher magnification images

Figure 3.3 (a) CVs of a non-activated sample (the arrow shows the direction of increasing scan rate). (b) The specific gravimetric and geometric capacitance of a non-activated sample at various scan rates

Figure 3.4 (a) CVs of microelectrodes in two-electrode cells after activation for $30 \mathrm{~min}$ at different scan rates (the arrow shows the direction of increasing scan rate). (b) typical CVs of samples before and after activation for 30 minutes in $0.5 \mathrm{M} \mathrm{H}_{2} \mathrm{SO}_{4}$ aqueous electrolyte at $100 \mathrm{mVs}^{-1}$ scan rate. (c) the specific gravimetric and geometric capacitance of activated samples at various scan rates.

Figure 3.5 Cyclic performance and capacity retention for an activated sample

Figure 3.6 (a) Galvanostatic charge/discharge curves at various discharge rates. (b) The specific gravimetric and geometric capacitance of activated samples at various discharge rates. The sample was electrochemically activated for $30 \mathrm{~min}$ 
Figure 4.1 (a) 2D and 3D design of interdigital electrodes. (b) Schematic 3D view of a PPy/C-MEMS micro-supercapacitor.

Figure 4.2 SEM images of (a) as-pyrolyzed C-MEMS electrodes (b) C-MEMS electrodes with PPy film. (c) High magnification image from the wall of a carbon post showing nanostructure of PPy film.

Figure 4.3 (a) CV curves of PPy/C-MEMS single electrodes with different electropolymerization time at $20 \mathrm{mVs}^{-1}$ scan rate. (b) Discharge curves of electrodes at 1 $\mathrm{mAcm}^{-2}$ discharge current density.

Figure 4.4 (a) Specific capacitance and (b) specific power of three single PPy/C-MEMS electrodes polymerized for 5,10 and 15 minutes at different scan rates.

Figure 4.5 Comparison of footprint normalized specific capacitance of PPy/2D carbon film and PPy/3D C-MEMS electrodes.

Figure 4.6 CVs of symmetric micro-supercapacitors with different polymerization time.

Figure 4.7 Galvonstatic charge/discharge curve of a symmetric micro-supercapacitor....69

Figure 4.8 (a) CV curves of a micro-supercapacitor with 15 min electropolymerization time at first and 1000th cycle (20 $\mathrm{mVs}^{-1}$ scan rate). (b) Cyclic performance of the device during $1000 \mathrm{CV}$ cycles

Figure 5.1 (a) Schematic drawing of ESD set-up that was used in this study. (b) Schematic drawing showing the deposited thin GNP film with open pore structure with easy accessibility of electrolyte ions

Figure 5.2 SEM image of the deposited GNP ( $6 \mu \mathrm{m}$ thick) film (a) cross section view (b) top view. (c) tem image of as received GNP.

Figure 5.3 Cyclic Voltammograms recorded in $1 \mathrm{M} \mathrm{Na}_{2} \mathrm{SO}_{4}$ (vs. $\mathrm{Ag} / \mathrm{AgCl}$ ) of GNP electrodes at various scan rates (a,b) $1 \mu \mathrm{m}$ thick electrode (c, d) $6 \mu \mathrm{m}$ thick electrode. (e) Plot of normalized capacitance vs. scan rate for electrode with 1 and $6 \mu \mathrm{m}$ thickness.....81

Figure 5.4 Galvonstatic charge/discharge curves of GNP thin film electrode at various current densities (a, b) $1 \mu \mathrm{m}$ thick electrode (c, d) $6 \mu \mathrm{m}$ thick electrode. (e) Specific capacitances obtained from galvanostatic charge/discharge measurements at various current densities. 
Figure 5.5 Plots of complex real $\left(\mathrm{C}^{\prime}\right)$ and imaginary $\left(\mathrm{C}^{\prime \prime}\right)$ capacitance as a function of frequency for (a) electrode with $1 \mu \mathrm{m}$ thickness (b) electrode with $6 \mu \mathrm{m}$ thickness. (c) Plots of utilizable specific energy vs. power density (Ragone plot) of the thin film GNP electrodes

Figure 6.1 FTIR absorbance spectra of (a) as-purchased go powder and (b) ESD deposited rGO.

Figure 6.2 Wide-range XPS spectra of (a) GO and (b) rGO. The C1s spectra were deconvoluted into their corresponding components using a Gaussian function for (c) GO and (d) rGO. .98

Figure 6.3 (a) Schematic drawing of fabrication procedures of micro-supercapacitors (inset shows a digital photograph of a fabricated device). (b) and (c) top view SEM micrographs of rGO-CNT based interdigital microelectrode arrays.

Figure 6.4 Scanning electron micrographs showing the morphology of the deposited electrodes: top view and tilted $35^{\circ}$ view of (a) and (b) rGO micro-electrodes, (c) and (d) rGO-CNT-9-1 micro-electrodes, and (e) and (f) rGO-CNT-8-2 micro-electrodes.

Figure 6.5 (A) CV curves at various cycles of a rGO micro-supercapacitors tested at 0.1 $\mathrm{Vs}^{-1}$ scan rate. (b) Variation of average $\mathrm{CV}$ current density with cycle number for $\mathrm{rGO}$, rGO-CNT-9-1 and rGO-CNT-8-2.

Figure 6.6 Schematic drawing showing the two mechanisms of increasing the ion accessibility of electrodes

Figure 6.7 CV curves of rGO, rGO-CNT-9-1 and rGO-CNT-8-2 micro-supercapacitors at $1 \mathrm{Vs}^{-1}$ scan rate .105

Figure 6.8 CV curves of rGO, rGO-CNT-9-1 and rGO-CNT-8-2 micro-supercapacitors at $5 \mathrm{Vs}^{-1}$ scan rate.

Figure $6.9 \mathrm{CV}$ curves of rGO, rGO-CNT-9-1 and rGO-CNT-8-2 micro-supercapacitors at $10 \mathrm{Vs}^{-1}$ scan rate

Figure 6.10 CV curves of rGO, rGO-CNT-9-1 and rGO-CNT-8-2 micro-supercapacitors at $25 \mathrm{Vs}^{-1}$ scan rate.

Figure 6.11 CV curves of rGO, rGO-CNT-9-1 and rGO-CNT-8-2 micro-supercapacitors at $50 \mathrm{Vs}^{-1}$ scan rate.

Figure 6.12 Comparison of stack capacitances of micro-supercapacitors with different electrode compositions. 
Figure 6.13 (a) Charge-discharge curves of micro-supercapacitors based on rGO, rGOCNT-9-1 and rGO-CNT-8-2 electrodes. (b) Specific capacitances of microsupercapacitor at different discharge current densities. (c) Nyquist plots of different micro-supercapacitors (inset shows the Nyquist plots at higher frequencies). (d) Phase angle vs. frequency for different micro-supercapacitor.

Figure 6.14 Ragone plot showing the relationship of specific energy and the specific power of micro-supercapacitors 


\section{INTRODUCTION}

\subsection{Overview}

Development of miniaturized electronic systems has stimulated the demand for miniaturized power sources that can be integrated into such systems. These micro-scale power sources should be able to provide enough energy and power for applications such as RFID tags, miniaturized MEMS devices in medical and pharmaceutical applications, and wireless sensors. For these applications, micro-batteries are considered to be the main candidates while the application of other micro power sources such as miniaturized energy harvesters and micro-fuel cells are emerging. However, micro-batteries share the drawbacks of their large counterparts and have a limited lifetime and low power density. Energy harvesters, on the other hand, still need an energy storage unit to store their excess energy and provide it when it is needed. Micro-supercapacitors can be paired with micro-batteries to provide peak power and improve the cycle lifetime or can be coupled with energy harvesting devices to store the generated energy. Moreover, microsupercapacitors with high energy density can potentially work as stand-alone and maintenance-free power sources for a number of applications.

The potential applications of micro-supercapacitors have attracted a lot of attention and there has been an exponential growth in the number of reports about microsupercapacitors in recent years. Most of the recent research efforts in the microsupercapacitor field have focused on increasing energy and power densities by improving the material properties and architecture of the devices. From the material aspect, various nanostructured materials have been utilized as electrode materials for microsupercapacitors. Several carbonaceous nanomaterials such as carbon nanotubes (CNT), 
activated carbons, carbide derived carbons, graphene, and carbon onions have been used to fabricate EDLC micro-supercapacitors. Moreover, transition metal oxides such as Ruthenium oxide and conductive polymers such as polypyrrole (PPy) have been used as materials for pseudo-capacitive micro-supercapacitors. From the device architecture aspect, there are mainly three types of micro-supercapacitor architectures reported in the literature so far. The first type is composed of thin film electrodes of nanostructured materials and these types of devices are fabricated by the two dimensional (2D) stacking of thin film electrodes and electrolytes. The second type of micro-supercapacitors consists of in-plane interdigital electrodes and offers various advantages over the conventional thin film devices. Recently, it has been shown that device performance can be improved by employing three dimensional (3D) electrodes with highly effective surface areas. However, more facile fabrication techniques are needed to expand the application of this type of micro-supercapacitor. Moreover, to meet the requirements of future applications, the performance of micro-supercapacitors in terms of their energy and power density should be improved.

\subsection{Motivation and Goal}

The major objective of this dissertation is to develop high power and high energy micro-supercapacitors. The future applications of micro-supercapacitors as miniaturized energy storage units for small and portable electronic devices depend on the improvement of their energy and power densities. Furthermore, reliable and scalable fabrication methods which are compatible with current manufacturing protocols of electronic devices should be developed. 
One of the approaches proposed in this dissertation is to improve the energy and power density of micro-supercapacitors by structuring the materials or current collectors into 3D structures with highly effective surface areas. To achieve this goal, carbonmicroelectromechanical systems (C-MEMS) is utilized in developing electrodes with 3D interdigital architecture. The 3D carbon microstructure can directly be utilized as electrodes for micro-supercapacitors. However, due to the relatively low specific surface area of as-prepared C-MEMS structures, efficient methods should be developed to activate these structures and increase their specific surface area. The current dissertation examines an electrochemical activation method to improve the performance of C-MEMS micro-supercapacitors.

In another approach, this dissertation proposes improving the energy density of micro-supercapacitors by utilizing C-MEMS structures as 3D current collectors for high energy density materials such as conducting polymers. To examine this approach, conformal deposition of polypyrrole films on carbon microstructures is studied and the micro-supercapacitors fabricated through this method were evaluated.

In another approach, this dissertation aims to develop ultra-high power microsupercapacitors with improved frequency response. The development of microsupercapacitors with high frequency response can potentially broaden the application of micro-supercapacitors by assuming the role of conventional electrolytic capacitors in applications such as ac line filtering. The development of ultra-high power microsupercapacitors has two major steps. The first step is to find the appropriate electrode materials and electrode fabrication technique which makes the maximum frequency response and rate handling ability of the electrode possible. The next step is to design 
micro-supercapacitor cells with improved electrolyte accessibility and decreased cell resistance to achieve capacitive behavior at ultra-high frequencies and charge/discharge rates. To achieve these goals, graphene and its composites with carbon nanotubes (CNTs) were utilized as capacitive materials for supercapacitor electrodes. Graphene with its exceptional properties such as chemical stability, high electrical conductivity, and large surface area has been proposed as a competitive material for supercapacitor application. A method based on electrostatic spray deposition (ESD) is proposed to fabricate thin films of graphene and graphene/CNT composites. Furthermore, a novel technique to fabricate micron-sized interdigital electrodes of graphene/CNT hybrid films is proposed. The micro-electrodes are designed to maximize the accessibility of electrolyte ions to the electrode and thus increase the energy and power density of micro-supercapacitors. Figure 1.1 presents the flow chart of the research plan.

\subsection{Scope of the Dissertation}

Chapter 2 provides the background of the dissertation and discusses the properties and applications of supercapacitors. The progress of micro-supercapacitors both from a materials point of view and an architectural view is discussed. Chapter 3 details the fabrication process and evaluation of 3D C-MEMS micro-supercapacitor. The studies on applications of electrochemical activation on improving the performance of microsupercapacitors are presented. Chapter 4 explains the advantages of using 3D carbon micro-structures as current collectors for high energy density capacitive materials. To demonstrate this concept, conformal deposition of polypyrrole (PPy) on carbon microstructures is investigated. This chapter includes the experimental results regarding optimum deposition conditions of PPy and electrochemical performance of PPy/C- 
MEMS micro-supercapacitors. Chapter 4 describes the fabrication of high power supercapacitor electrodes by electrostatic spray deposition of graphene nanoplatelets. The effect of electrode thickness on the performance of the electrodes was also studied and presented in this chapter. Chapter 5 explains design, fabrication and evaluation of ultrahigh power micro-supercapacitor based on graphene/ CNT hybrid electrodes. The simultaneous deposition and reduction of graphene oxide precursor materials is explained and a novel method to integrate graphene films on gold interdigital micro-electrodes is introduced. This chapter also explains the addition of CNTs as nano spacers between graphene sheets and their effects on the performance of micro-supercapacitors. Chapter 6 provides a summary of the dissertation and proposes future works.

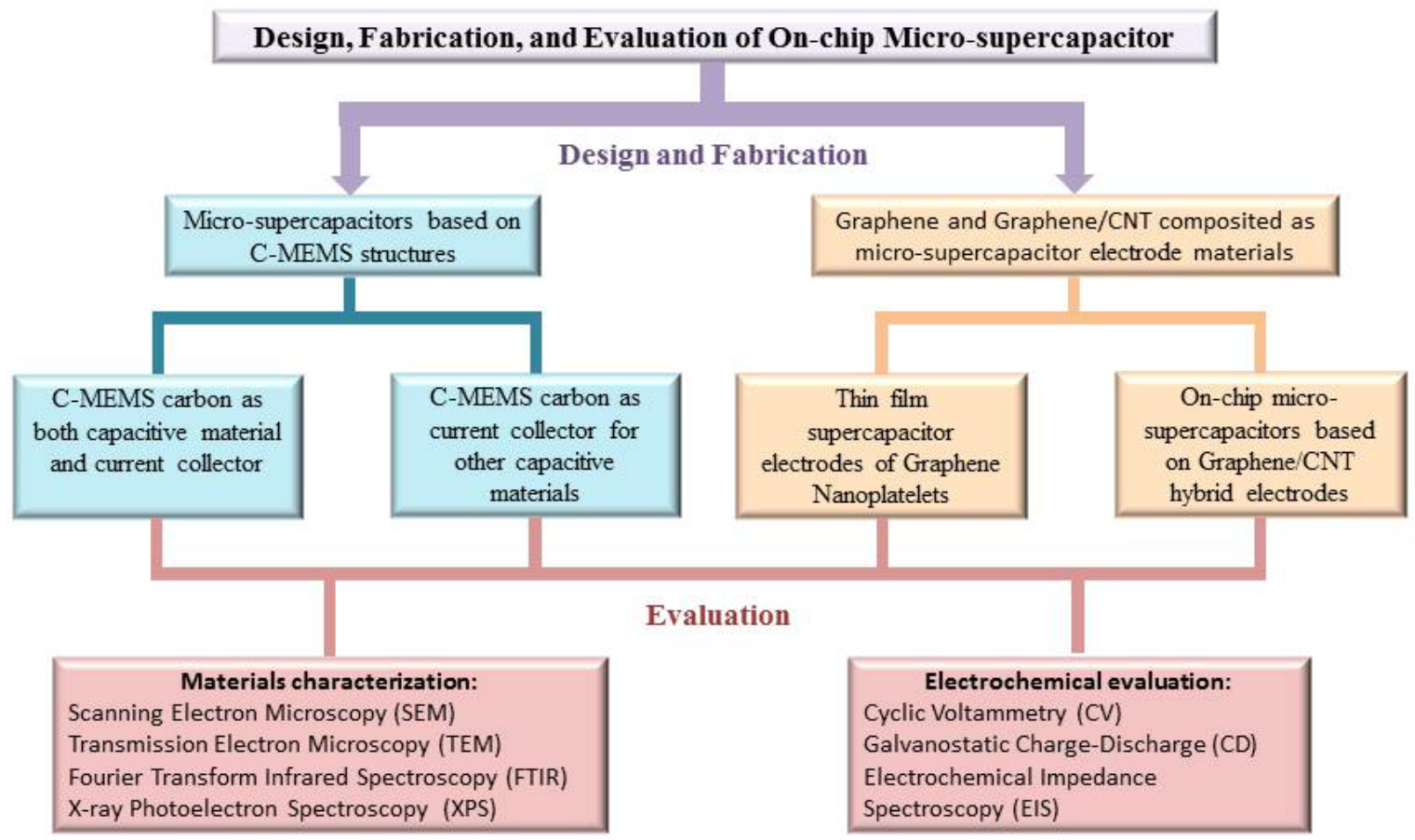

Figure 1.1 Flow chart of the research plan. 


\section{BACKGROUND AND LITERATURE REVIEW}

\subsection{Introduction}

In recent years, decreased availability of fossil fuels and the problems associated with climate change have motivated a growing interest in the production and utilization of renewable and sustainable energy from resources such as sun and wind. However, to use the energy produced from these resources when and where we need it, this energy must be stored in appropriate energy storage devices. At the forefront of these are electrical energy storage devices [1]. Batteries and electrochemical capacitors (ECs) (also known as supercapacitors or ultra-capacitors) are two important examples that have had a growing impact in our lives by storing energy in applications ranging from portable electronics to electric and hybrid vehicles and large industrial equipment. For more than a decade Li-ion batteries and other types of secondary batteries have been a focus of research to improve their performance to meet the requirements of more challenging applications. The use of nanomaterials as battery electrodes along with advances in fabrication methods, electrolytes and packaging, has resulted in improved performance of batteries [2]. However, because batteries have relatively slow power delivery and uptake, there is a need for fast and high power energy systems in a number of applications [1]. This need has motivated the research on supercapacitors. The earliest patents on supercapacitors were filed in the 1950s, however in the 1990s supercapacitors attracted a lot of attention as it was found that a major function of supercapacitors could be to complement battery and fuel cells in a hybrid vehicle and provide the necessary power during acceleration or to recuperate brake energy [3,4]. Further developments led to recognizing supercapacitors as the devices that can bridge the gap between conventional 
capacitors which have high power but low energy and batteries and fuel cells which have high energy but relatively low power density $[1,3,4]$. Figure 2.1 illustrates this concept in a so called "Ragone plot" in terms of specific power and specific energy of different energy storage devices. In terms of both specific power and specific energy the gap between conventional capacitors and batteries covers several orders of magnitude and can be filled by electrochemical capacitors. The US department of energy has designated electrochemical capacitors as important as batteries for the future of electrical energy storage [5].

In recent years, there has been a boost in the field of supercapacitors with a lot of theoretical and practical research efforts focusing on improvement of supercapacitor properties and overcoming the major challenge of these devices which is low energy density.

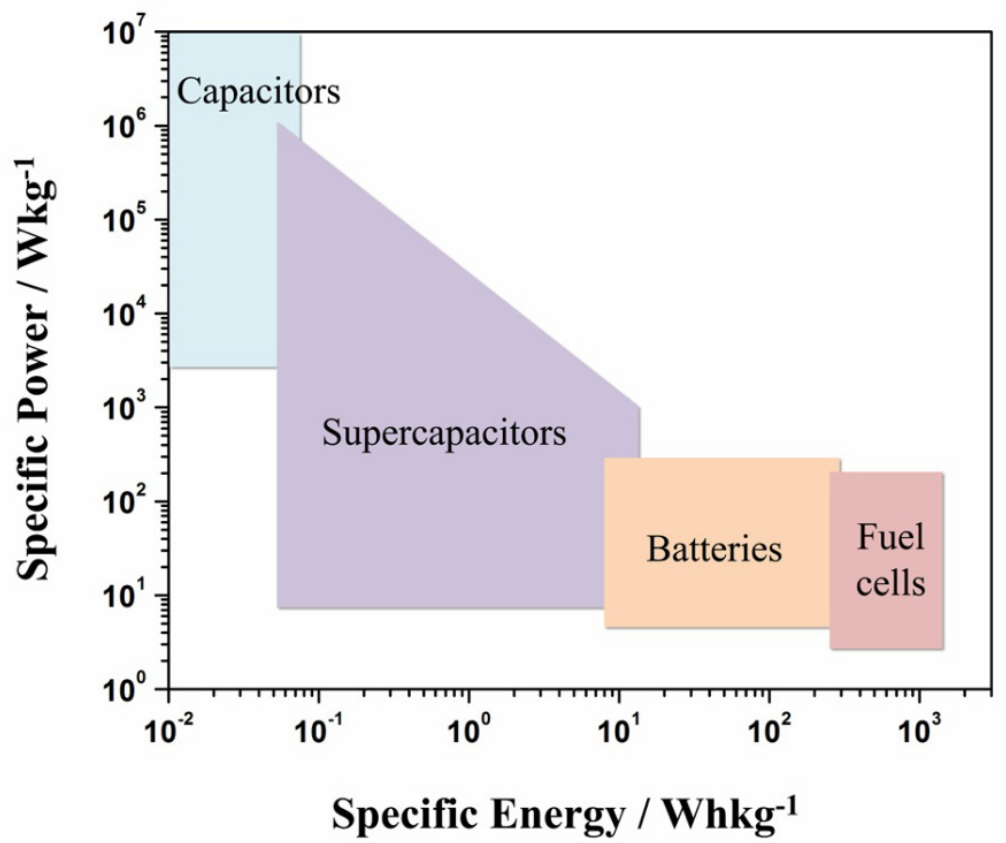

Figure 2.1 Ragone plot of specific power versus specific energy for various electrical energy storage and conversion devices. Adapted from Ref. [4]. 


\subsection{Historical Background}

The first electrochemical charge storage devices using double layer charge storage was patented by Becker [6] from General Electric company in 1957. However, it was not before 1966 that it was understood that the charge storage mechanism is based on the electrical double layer at the electrode/electrolyte interface. In 1978, the Nippon Electric Company (NEC) developed the first commercial electrochemical capacitor and used the term "supercapacitor" for the developed device. Around the same time, Panasonic developed its own commercial supercapacitor called "Goldcap". The early application of electrochemical capacitors was restricted to replacing batteries for memory back-ups. In the late 1970s, Professor Conway from university of Ottawa carried out extensive research on another mechanism for electrochemical capacitors. This charge storage mechanism was based on a faradic reaction on the surface of the electrode materials and was termed as pseudo-capacitance. Conway's preliminary researches were based on pseudo-capacitance associated with solid oxide redox system, especially in $\mathrm{H}_{2} \mathrm{SO}_{4}$ electrolyte and for $\mathrm{RuO}_{2}$ [7]. Due to increased amount of possible applications, the whole field of electrochemical capacitors flourished in the 1990s and significant amount of research efforts were focused on improvement of supercapacitor properties. In the present days, several other companies including Maxwell technologies, NNC, Cap-XX, AVX, Cooper, ELNA, and several others have invested in the development of supercapacitors.

\subsection{Electrochemical Properties of Supercapacitors}

Electrochemical properties of supercapacitors can be explained by their capacitance, voltage, power and energy density. The two electrodes of a supercapacitor cell can be assumed to be two capacitors in series. Now if the capacitance of the two electrodes were 
expressed as $C_{p}$ and $C_{n}$ for the positive and negative electrodes, respectively, then the total capacitance of the cell (CT) can be expressed by equation (2.1) [8]:

$$
\frac{1}{C_{T}}=\frac{1}{C_{p}}+\frac{1}{C_{n}}
$$

If the two electrodes are the same and have the same weight, it can be assumed that they have equal capacitances $\left(\mathrm{C}_{\mathrm{p}}=\mathrm{C}_{\mathrm{n}}\right)$. Then the total capacitance would be half of the capacitance of one of the electrodes. The unit of capacitance is Farads (F), but the capacitance of a supercapacitor is commonly reported in terms of specific capacitance which is the capacitance normalized by weight, surface area or volume of the cell or a single electrode. The most common way of reporting the specific capacitance is reporting the specific capacitance of a single electrode which can determine in laboratory scale electrochemical cells with three electrodes. In a three-electrode cell a single supercapacitor electrode would be the single electrode and the two other electrodes are counter and reference electrodes.

In general, the capacitance depends on the amount of charge stored on the electrodes, which in turn depends on the properties of the electrode materials. When a supercapacitor is charged, a voltage (V) will build up across the two electrodes. Maximum energy (E) and power $(\mathrm{P})$ densities of supercapacitors can be determined by equations (2.2) and (2.3) [3,9]:

$$
\begin{aligned}
& E=\frac{1}{2} C V^{2}=\frac{Q V}{2} \\
& P=\frac{1}{4 R_{s}} V^{2}
\end{aligned}
$$


where $\mathrm{Q}$ is the total charge stored in the supercapacitor and $\mathrm{R}_{\mathrm{S}}$ is the equivalent inner resistance of the cell. Power and energy density of supercapacitors can be calculated by normalizing equations (2.2) and (2.3) by mass, surface area or volume of the device or a single supercapacitor electrode. As it is evident by these equations, the energy and power density of supercapacitors can be increased by increasing the capacitance or working voltage of the device, or by lowering $R_{s}$. The value of capacitance (C) is mainly a function of electrode materials' properties. For instance, the capacitance of carbon materials is dependent on their intrinsic capacitance and also their electrolyte accessible surface area.

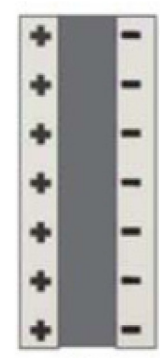

(a)

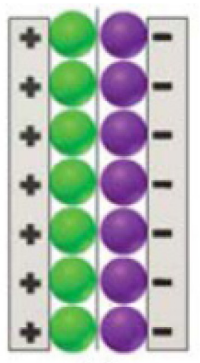

(b)

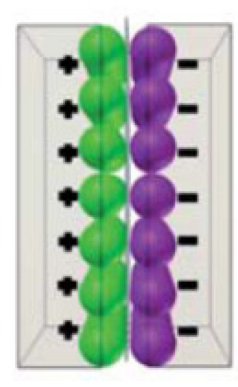

(c)

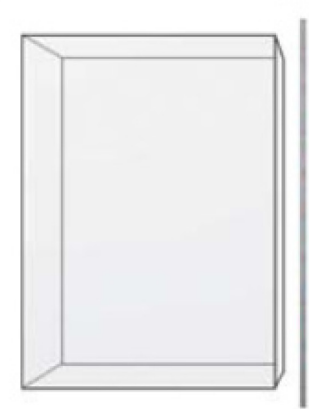

(d)

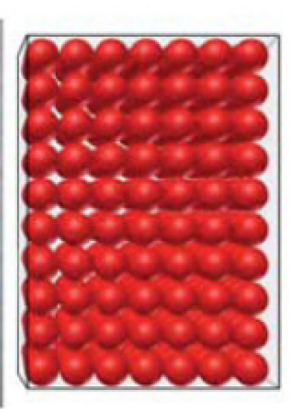

Figure 2.2 Representation of charge storage mechanisms in (a) a conventional capacitor, (b) EDLC, (c) pseudo-capacitor, and (d) a battery. Adapted from Ref. [10].

The working voltage of a supercapacitor is dependent on the electrode material and the electrolyte. For example, for carbon electrodes and aqueous electrolytes, the cell voltage is about $1 \mathrm{~V}$ and at a higher voltage the electrolyte would not be stable. However, 
the same carbon electrode may show a working voltage in the range of $3-3.5 \mathrm{~V}$, if an organic electrolyte is used. Moreover, equation (2.3) shows that the power of supercapacitors can be increased by decreasing the internal resistance of the cell $\left(R_{s}\right)$. Internal resistance is a sum of the electrode and electrolyte resistances and it can be decreased by high conductivity electrode materials and electrolytes. Moreover, designing the electrodes in such a way that decreases the diffusion path of ions can also decrease $\mathrm{R}_{\mathrm{s}}$.

\subsection{Charge Storage Mechanisms of Supercapacitors}

EDLCs store charge electrostatically on the electrode/electrolyte interface of high surface area materials, and pseudo-capacitors or redox capacitors store charge in the bulk of a redox material in response to fast surface and near-surface redox reactions [1,7].

In other words, in contrast to conventional capacitors where charge storage mechanisms are the physical separation of charge on the electrode by a dielectric material (Figure 2.2a), in ESLCs charge separation occurs in a double layer formed on an electrode/electrolyte interface (Figure 2.2b). In pseudo-capacitors, charge transfer reactions occur at the surface or sub-surface of the electrode materials (Figure 2.2c). The charge storage mechanism of supercapacitors differs from that of batteries (Figure 2.2d) where a change in oxidation state of the bulk of the electrode material results in storing energy as chemical potential [10].

\subsection{Electrode Materials for Supercapacitors}

In this section, the common materials for two main categories of electrochemical capacitors, EDLCs and pseudo-capacitors will be briefly discussed. As it was mentioned

previously, there is no faradic reaction in EDLCs and electric charge is stored by aggregation of charges at the surface of the electrode materials. Therefore, an important 
principal for high performance EDLCs is the use of electrochemically stable electrode materials with high surface area and conductivity $[1,11]$. Conductive carbon materials satisfy all these requirements and are the most explored candidates in this application due to their low cost, process ability, stability and electrical conductivity. Hence, a main focus of the research in the EDLC field is to develop carbon-based electrode materials with large accessible surface areas $[1,11]$. Development of different kinds of high specific surface area carbon materials has improved the capacitive properties of EDLCs. Porous carbon materials such as activated carbons (ACs), template carbons and carbide derived carbons [9], one-dimensional carbon materials such as carbon nano fibers and carbon nanotubes (CNTs) [12], and most recently two-dimensional graphene sheets [9] are among the long list of carbon nanomaterials that are used as materials for EDLC electrodes. Due to their high surface area, low cost and relatively good electrical properties, ACs are the most widely used materials for supercapacitor electrodes [13]. Production of ACs usually follows physical, chemical or electrochemical activation of the carbonaceous materials. In physical activation, carbon precursors are treated at high temperatures ranging from 700 to $1200{ }^{\circ} \mathrm{C}$ in the presence of oxidizing agents such as steam, $\mathrm{CO}_{2}$ and air [13]. Chemical activation involves the use of activation agents such as potassium hydroxide, zinc chloride, sodium hydroxide, and phosphoric acid and treatment at temperatures ranging from 400 to $700{ }^{\circ} \mathrm{C}$. Electrochemical activation is usually performed by oxidation and reduction of carbon electrodes in an electrochemical cell. ACs with different properties with surface areas as high as $3000 \mathrm{~m}^{2} \mathrm{~g}^{-1}$ have been produced and were studied for their electrochemical properties [13-18]. Although ACs have a high surface area, control of their pore size distribution and pore structure is still a 
challenge [13]. Designing ACs with well controlled interconnected pore structure could effectively improve the energy and power density of AC based supercapacitors.

Another type of carbon that is used for supercapacitor applications is templatederived carbon. In recent years, there have been remarkable advances in fabrication of carbons with ordered porous structure through tempting methods $[13,19]$. The fabrication process is commonly conducted through infiltration of carbon precursors in pores of an inorganic template followed by a carbonization treatment and removal of the template. Compared to ACs, template-derived carbons offer a high specific surface area with regularly interconnected pores that allow for improved ionic transport. Therefore, supercapacitors based on these materials show higher power density compared to ACbased supercapacitors. As for their performance, template-derived carbon with surface areas of $1500-1600 \mathrm{~m}^{2} \mathrm{~g}^{-1}$, exhibit a specific capacitance of $220 \mathrm{Fg}^{-1}[10,20]$.

Another type of carbon materials that have attracted a lot of attention for supercapacitor applications are CNTs [21-25]. This is due to their good electrical properties, unique pore structure and superior thermal and mechanical stability. The electrical properties and open pore structure of CNTs make them an excellent choice for high power supercapacitor electrodes. However, the energy density of CNT electrodes are immensely effected by their relatively low surface area $\left(<500 \mathrm{~m}^{2} \mathrm{~g}^{-1}\right)$. Specific capacitance of between 15 and $300 \mathrm{Fg}^{-1}$ have been reported for different configurations of CNTs [10].

Other types of carbon such as CDCs are also under extensive research for application as supercapacitor electrode materials. CDCs are manufactured by chlorination of metallic carbides such as $\mathrm{TiC}, \mathrm{SiC}, \mathrm{B}_{4} \mathrm{C}$. 
Table 2.1 Properties and performance of various carbon-based supercapacitor electrodes. Adapted from Ref. [13].

\begin{tabular}{|c|c|c|c|c|c|c|}
\hline \multirow[b]{2}{*}{ Materials } & \multirow[b]{2}{*}{$\begin{array}{l}\text { Specific Surface } \\
\text { area/ } \mathbf{m}^{2} \mathbf{g}^{-1}\end{array}$} & \multirow[b]{2}{*}{$\begin{array}{l}\text { Density/ } \\
\text { gcm }^{-3}\end{array}$} & \multicolumn{2}{|c|}{$\begin{array}{c}\text { Aqueous } \\
\text { electrolyte }\end{array}$} & \multicolumn{2}{|c|}{$\begin{array}{c}\text { Organic } \\
\text { electrolyte }\end{array}$} \\
\hline & & & $\mathrm{Fg}^{-1}$ & $\mathrm{Fem}^{-3}$ & $\mathrm{Fg}^{-1}$ & $\mathrm{Fcm}^{-3}$ \\
\hline $\begin{array}{l}\text { Commercial activated } \\
\text { carbons (ACs) }\end{array}$ & $1000-3500$ & $0.4-0.7$ & $<200$ & $<80$ & $<100$ & $<50$ \\
\hline $\begin{array}{l}\text { Particulate carbon from } \\
\mathrm{SiC} / \mathrm{TiC}\end{array}$ & $1000-2000$ & $0.5-0.7$ & $170-220$ & $<120$ & $100-120$ & $<70$ \\
\hline $\begin{array}{l}\text { Functionalized porous } \\
\text { carbons }\end{array}$ & $300-2200$ & $0.5-0.9$ & $150-300$ & $<180$ & $100-150$ & $<90$ \\
\hline Carbon nanotube (CNT) & $120-500$ & 0.6 & $50-100$ & $<60$ & $<60$ & $<30$ \\
\hline $\begin{array}{l}\text { Templated porous carbons } \\
\text { (TC) }\end{array}$ & $500-3000$ & $0.5-1$ & $120-350$ & $<200$ & $60-140$ & $<100$ \\
\hline $\begin{array}{l}\text { Activated carbon fibers } \\
\text { (ACF) }\end{array}$ & $1000-3000$ & $0.3-0.8$ & $120-370$ & $<150$ & $80-200$ & $<120$ \\
\hline Carbon cloth & 2500 & 0.4 & $100-200$ & $40-80$ & $60-100$ & $24-40$ \\
\hline Carbon aerogels & $400-1000$ & $0.5-0.7$ & $100-125$ & $<80$ & $<80$ & $<40$ \\
\hline
\end{tabular}

The resulting carbon materials exhibit a nanoporous structure with narrow pore size distribution and it is possible to control their pore size by selection of different carbides as the starting material and controlling the chlorination temperature [26]. Specific capacitances in the range of 140 to $260 \mathrm{Fg}^{-1}$ have been reported for CDC supercapacitor electrodes depending on the precursor carbide and chlorination temperature of carbide and the electrolytes that were used to test the supercapacitors [26]. The mechanism of charge storage in the pseudo-capacitor is by faradic or redox reactions that take place on the surface of capacitive materials resulting in faradic current passing through the supercapacitor cell [3]. Pseudo-capacitive materials include conducting polymers such as polyaniline, polypyrrole, polythiophene and their derivatives and also metal oxides such as $\mathrm{RuO}_{2}, \mathrm{MnO}_{2}$ and $\mathrm{Co}_{3} \mathrm{O}_{4}[1,3,7,27,28]$. This faradic reaction results in higher specific capacitance of pseudo-capacitors compared to EDLCs. However, since 
these faradic processes are generally very slow, pseudo-capacitors like batteries suffer from relatively low power density compared to EDLCs.

Metal oxides that can be used for supercapacitor applications should be electronically conductive and should have two or more oxidation states that exist over a continuous potential range without irreversible phase and structure changes [1,3]. Ruthenium oxide is the one widely studied metal oxide for supercapacitor application because it has good electronic conductivity and also has three distinct oxidation states in a $0-1.2 \mathrm{~V}$ voltage range [1]. The behavior of $\mathrm{RuO}_{2}$ in acidic electrolytes involve rapid and reversible electron transfer along with electro-adsorption of protons on the surface of $\mathrm{RuO}_{2}$ particles according to equation (2.4), where $\mathrm{Ru}$ oxidation states can change from $\mathrm{Ru}(\mathrm{II})$ to $\mathrm{Ru}(\mathrm{IV})$. $\mathrm{RuO}_{2}+x \mathrm{H}^{+}+x e^{-} \leftrightarrow R u \mathrm{O}_{2-x}(\mathrm{OH})_{x}$

where $0 \leq x \leq 2$. Specific capacitance of as a high as $600 \mathrm{Fg}^{-1}$ for ruthenium oxide and as high as $1200 \mathrm{Fg}^{-1}$ for ruthenium based composite electrodes are reported [1,3]. However, ruthenium oxide is an expensive material and its $1 \mathrm{~V}$ potential window limits the application of supercapacitors based on $\mathrm{RuO}_{2}$ to small electronic devices. Other oxides, such as oxides of manganese, iron, nickel and cobalt have been extensively researched for supercapacitor electrode application. Figure 2.3 summarizes the specific capacitances reported for different supercapacitor materials in the literature.

A hybrid supercapacitor with asymmetrical electrodes contains a carbon based EDLC electrode and an electrode based pseudo-capacitor material. These types of devices take advantage of both the charge storage mechanism to improve the energy and the power density of supercapacitor cells and have been extensively studied in recent years [30-32]. 


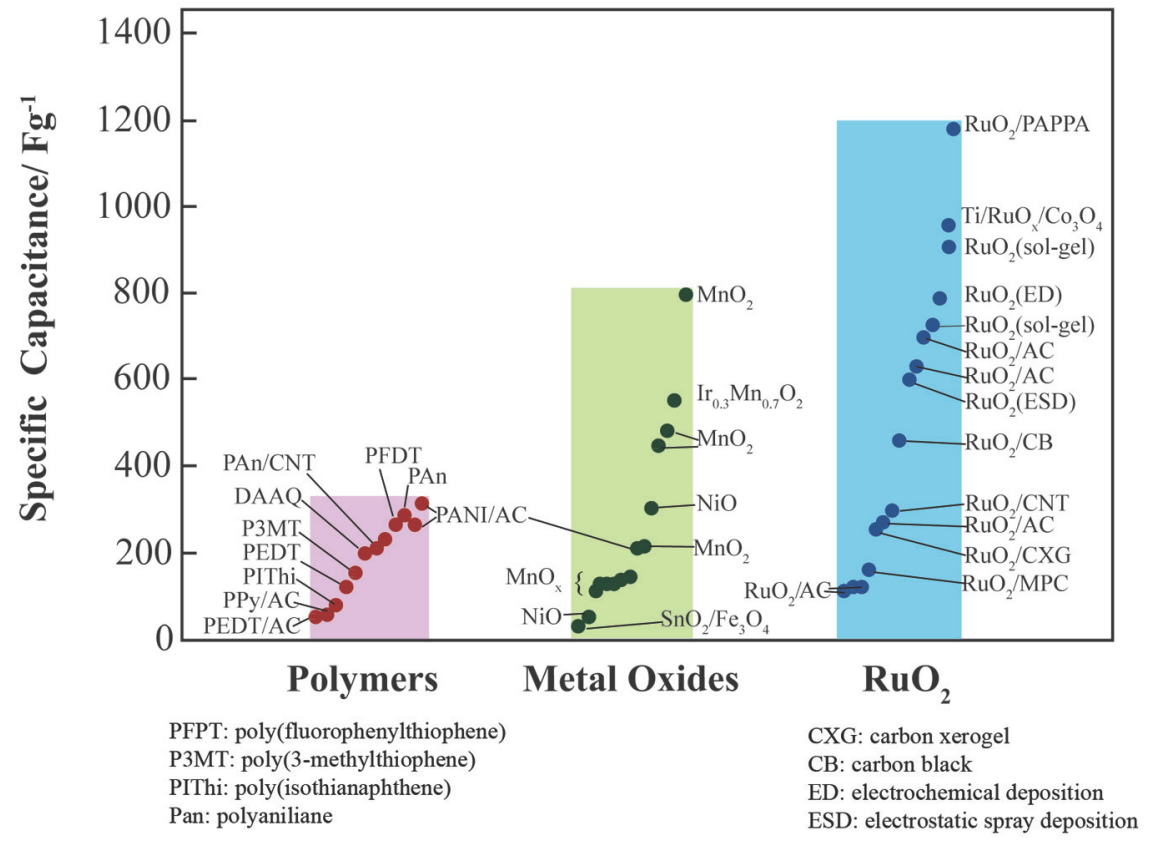

Figure 2.3 Specific capacitance reported for various supercapacitor electrode materials. Adapted from Ref. [29].

\subsection{Miniaturized Power Sources}

Advancements in the field of micro and nano-scale fabrication have inspired development of micro-power sources which are potentially able to be integrated into small electronic devices. Two types of microdevices have been studied to generate power for microsystems. The first type includes devices that have a finite amount of energy or require a continuous supply of active material or fuel for power generation [33]. Microbatteries [34], micro-combustors [35], micro-heat engines [36], and micro-fuel cells [37] are devices that fall in this category. The second type of devices are based on energy harvesting which extract energy from the ambient environment. Some of the typical devices in this category include micro-thermoelectric devices [38], microelectromagnetic devices [39], micro-thermophotovoltaic devices [40], and micro- 
piezoelectric devices [41]. Among these, micro-electrochemical energy storage and conversion devices such as batteries, fuel cells and supercapacitors are particularly attractive because of (i) their higher efficiency compared to internal or combustion engines; (ii) their relatively high energy density; and (iii) their advantages related to their operation such as low-vibration, low-noise, and negligible air pollution [42]. Thin film micro-batteries are the most widely researched devices for use as integrated micro power sources. A micro-battery works by the same principal as classical batteries; it usually consists of two electrodes, i.e., an anode and a cathode, which are separated by an electrolyte layer with or without a separator.

In general, batteries have certain drawbacks such as limited lifetime and lower power density compared to other power systems. The finite life time of micro-batteries limits their application where battery change is not possible or practical [34]. Moreover, the low power density of micro-batteries is insufficient for some applications that require high power in a short timeframe. Other types of micro power sources also have some limitations. Micro-fuel cells such as batteries are suffering from low discharge rate and low power density, a limitation that they also share with some of the energy harvesters. Another issue with energy harvesters is the energy that they generate should be consumed immediately or it will be lost. As a result, these devices are usually accompanied by energy storage devices to store the excess generated energy and provide it when it is needed.

Micro-supercapacitors can solve many problems associated with other micro power sources or miniaturized energy storage devices. As a stand-alone power source, high power density micro-supercapacitors are able to satisfy the power needs of certain 
miniaturized electronic devices. For instance, they can power radio frequency identification (RFID) tags which generally require $1-100 \mu \mathrm{W}$ power [43]. Moreover, micro-supercapacitors can also play the role that their large counterparts play for large batteries. Micro-supercapacitors can be coupled with micro-batteries to provide the peak

power and improve their lifetime. Additionally, higher power densities, faster charge/discharge capability and longer cycle lifetime micro-supercapacitors make them an excellent choice energy storage to store the excess energy of miniaturized energy as harvesters.

\subsection{On-chip Micro-supercapacitors}

Due to the increasing demand for high power and reliable miniaturized energy storage devices, development of micro-supercapacitors or electrochemical microcapacitors have attracted a lot of attention in recent years. The first generation of microsupercapacitor reported in the early 2000s were fabricated by thin film technologies. However, further development of micro-supercapacitors was also realized in other device architecture. In general, based on their design, micro-supercapacitors can be categorized into three categories. These categories are briefly introduced and compared in the following section.

\subsubsection{Architecture of Micro-supercapacitors}

Charge-transfer reactions of a matter are the key element of all electrochemical phenomena. The main parts of all electrochemical storage devices are two opposing electrodes which are commonly referred to as anode and cathode. This simple device concept becomes more complicated if we want to achieve optimal mobility of reactant, ions and electrons. It is well-known that the performance of electrochemical devices can 
be improved by optimizing the electrode material properties. For example, electrochemical properties of supercapacitor electrodes depend on utilizing high surface area materials that are engineered to have maximum accessibility. Ions should be able to easily access the whole surface of the electrode materials and electrons should be able to move to the current collectors with minimum resistivity. In recent years, improving the device performance by optimizing the electrode configurations and architecture of the electrochemical devices has also attracted a lot of attention.

The first generation of micro-supercapacitors followed the architecture of thin film batteries which was the two-dimensional (2D) stacking of the device components. In this design thin layers of an anode, solid electrolyte and a cathode are stacked on top of each other to make up the basic structure of the device (Figure 2.4a). Although the simplicity of production of $2 \mathrm{D}$ devices decreases the production costs for mass production, these structures cannot fully satisfy the main challenge of micro power sources, which is providing the maximum power in the limited footprint area of the device [44].

Advancement in this field has led to realizing three-dimensional (3D) structures by complex non-planar configuration of electrode materials to increase the energy and power density of cells within the footprint area. A 3D arrangement of the electrodes could decrease the transport path of ions between the electrode, and at the same time increase the energy density [44]. There are several possibilities to arrange the device component in a 3D structure. The simplest 3D structures that have been realized for microbatteries are schematically shown in figure $2.3 \mathrm{c}$. 


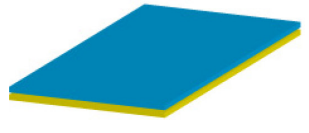

(a)

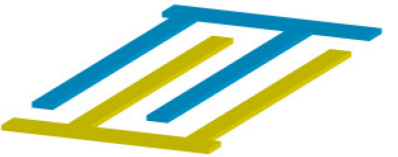

(b)

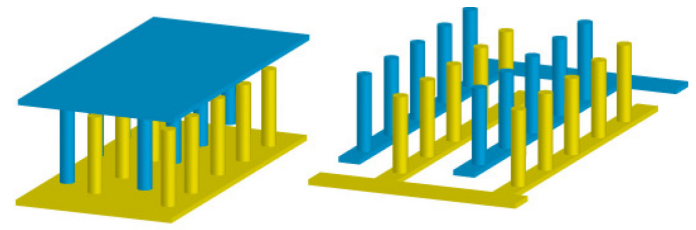

(c)

Figure 2.3 Three different device architectures for on-chip micro-supercapacitors. (a) 2D planar, (b) in-plane interdigital, and (c) two examples of possible 3D designs.

For both of these structures, each electrode consists of several micro-electrodes with small spacing between the electrodes. In one of the architectures that are shown in figure 2.4c, 3D anode and cathodes are fabricated on separate substrates and are assembled in an interdigitated structure. In the other possible architecture, the interdigital anode and cathode are fabricated on the same substrate. Both of these architectures offer the advantages of 3D design of electrodes by minimizing the diffusion path of electrolyte ions between the electrodes and also increasing the effective surface area of each electrode in the footprint area of the device.

To demonstrate the increase in effective surface area of 3D architecture compared to a $2 \mathrm{D}$ planar device, consider a simple 3D design in which the posts in figure $2.3 \mathrm{c}$ have a radius of $20 \mu \mathrm{m}$ and are separated by $20 \mu \mathrm{m}$ ( $40 \mu \mathrm{m}$ center to center). These dimensions for the 3D structure are achievable by several MEMS fabrication methods. Assuming a 1 $\mathrm{cm}^{2}$ footprint area for both $2 \mathrm{D}$ and $3 \mathrm{D}$ design, then the $2 \mathrm{D}$ design the effective surface area of anode and cathode are equal to $1 \mathrm{~cm}^{2}$. For the 3D design, however, assuming $\mathrm{L}$ (the height of the posts) to be $200 \mu \mathrm{m}$, the calculated surface area of the anode and cathode would be $\sim 7.5 \mathrm{~cm}^{2}$ each. If $\mathrm{L}$ increases to $500 \mu \mathrm{m}$ the surface area of anode and cathode would be $\sim 19.5 \mathrm{~cm}^{2}$ each. This cell consists of a total of 62500 anodes and 
cathodes (31250 anode and 31250 cathodes) and has a total of $\sim 39 \mathrm{~cm}^{2}$ active materials. If $\mathrm{L}$ becomes large, it can be assumed that the ohmic resistance of the electrodes becomes sufficiently large to offset the advantages of increased areal capacity [44]. The optimized value of $\mathrm{L}$ depends on a combination of parameters including electronic and ionic conductivity of the electrode materials, the ionic conductivity of the electrolyte, and the geometry of the electrodes [46].

\subsubsection{Thin Film Micro-supercapacitors}

There are numerous reports on thin film electrodes for supercapacitor application in the literature. Both EDLC and pseudo-capacitor materials as well as their composites are used to fabricate electrodes for thin film capacitors. Fabrication methods of thin film electrodes include sputtering, electrodeposition, chemical vapor deposition (CVD), inkjet printing, layer by layer deposition, electrospray deposition and several other methods. In this section, some of the reported thin film supercapacitors will be reviewed. The emphasis will be on the reports that describe the full device fabrication.

Lim et al.[47] reported on the earliest work on thin film supercapacitors, a device that was based on two thin films of pseudo-capacitive ruthenium oxide $\left(\mathrm{RuO}_{2}\right)$ and Lipon solid electrolyte. The fabricated full cell delivered a volumetric capacitance of about $38 \mathrm{mFcm}^{-2} \mu \mathrm{m}^{-1}$, however its capacitance dropped by $53 \%$ after only 500 cycles. Kim et al. [48] developed a thin film supercapacitor with similar design but using radio frequency sputtered cobalt oxide $\left(\mathrm{Co}_{3} \mathrm{O}_{4}\right)$ as the electrode material and with comparable performance with the device based on $\mathrm{RuO}_{2}$. White et al. [49] reported a polymer based thin film supercapacitor. This device based on polypyrrole electrodes doped with 12molybdosilicic acid showed a specific capacitance of $33.4 \mathrm{Fg}^{-1}$. The relatively short 
lifetime of thin film supercapacitors based on pseudo-capacitive materials is their main drawback that limits their application. Recently, the main focus has been EDLC thin film supercapacitor based on carbon materials such as CNT and graphene. A device based on printed single walled CNT thin films were reported by Kaempgen et al. [50]. This thin film supercapacitor showed an estimated capacitance of $1.1 \mathrm{mFcm}^{-2}$, in a potential window of 0 to $1 \mathrm{~V}$. The utilization of carbon materials, introduced the possibility of fabrication of flexible supercapacitor devices, adding another functionality to thin film supercapacitors. For example, $\mathrm{Hu}$ et al. [51] reported a flexible supercapacitor device which consists of CNT coated paper electrodes. This device showed a specific capacitance of $3 \mathrm{mFcm}^{-2}$.

\subsubsection{Micro-supercapacitors with In-plane Interdigital Electrodes}

One of the first micro-supercapacitors with interdigital in-plane electrodes was reported by Sung et al. [52]. They first deposited Polypyrrole (PPy) electrode materials on interdigital gold electrodes fabricated on a silicon substrate and then attached a sheet of solid electrolyte prepared from mixing polyvinyl alcohol (PVA) and phosphoric acid $\left(\mathrm{H}_{3} \mathrm{PO}_{4}\right)$ on the prepared interdigital electrode. Upon detaching the polymer electrolyte from the silicon substrate, a flexible micro-supercapacitor with PPy electrodes was achieved. The device showed good capacitive performance at a scan rate of $100 \mathrm{mVs}^{-1}$, but the ideal capacitive behavior was not observed by increasing the scan rate to a moderate scan rate of $500 \mathrm{mVs}^{-1}$. The authors did not report the specific capacitance and energy and power density of the device [52]. In another study, Jiang et al. [53] fabricated micro-supercapacitors with interdigital carbon forest electrodes. The electrodes were fabricated by a combination of standard lithography methods to fabricate metallic current 
collectors and CVD growing of vertically aligned CNT forest. This CNT-based microsupercapacitor showed a specific capacitance of $428 \mu \mathrm{Fcm}^{-2}$. Pech et al. [54] reported on fabrication of on-chip micro-supercapacitors by ink-jet printing of AC on micro-patterned gold electrodes. The device showed medium specific capacitance $\left(0.4 \mathrm{mFcm}^{-2}\right.$ average capacitance of the cell), however, due to the use of polymeric binders and limited ion transfer in the porous network of the electrode materials, AC micro-supercapacitors show relatively poor frequency response [54]. In 2010, Chmiola et al. [45] reported on microsupercapacitors based on monolithic carbide-derived carbon (CDC) interdigital electrodes. The electrodes were made by selective etching of monolithic CDC film using gold electrode both as a mask for etching and the current collectors. The process was later improved by Heon et al. [55] and the developed micro-supercapacitors showed high volumetric capacitance at low scan rates (about $180 \mathrm{Fcm}^{-3}$ volumetric capacitance of one electrode at $20 \mathrm{mVs}^{-1}$ ), however, the capacitance dropped to almost half of its initial value by increasing the scan rate to $500 \mathrm{mVs}^{-1}$, suggesting the poor rate handling capability of these micro-supercapacitors [55]. Pech et al. [56] reported fabrication and test of a micro-supercapacitor based on onion-like carbon (OLCs). The OLC based micro-supercapacitors are particularly notable as they offer ultra-high power handling capability with a resistance capacitance (RC) time constant of only $26 \mathrm{~ms}$. The combination of micrometer-sized interdigital electrode design with a binder free deposition technique and the non-porous morphology of OLC materials was responsible for the excellent frequency response of OLC based micro-supercapacitors. The drawback of OLC based micro-supercapacitors is their modest specific capacitance $\left(1.7 \mathrm{mFcm}^{-2}\right)$ and their high temperature processing requirements $\left(\sim 1800{ }^{\circ} \mathrm{C}\right)$ [56]. Gao et al. [57] 
reported a direct write process to fabricate micro-supercapacitors with interdigital electrode design and hydrated graphene oxide (GO) as the electrolyte and separator. While the fabrication method is promising, the electrodes demonstrated in this work were fabricated in millimeter scales with the maximum capacitance of only $0.51 \mathrm{mFcm}^{-2}$ for an in-plane design of electrodes.

Table 2.2 Summary of reported micro-supercapacitors with interdigital in-plane electrodes.

\begin{tabular}{|c|c|c|c|c|c|}
\hline $\begin{array}{l}\text { Electrode } \\
\text { material }\end{array}$ & Electrolyte & $\begin{array}{l}\text { Potential } \\
\text { range } \\
\text { (V) }\end{array}$ & $\begin{array}{l}\text { Specific } \\
\text { capacitance } \\
\left(\mathrm{mFcm}^{-2}\right)\end{array}$ & $\begin{array}{l}\text { Frequency response } \\
\text { and rate capability }\end{array}$ & Reference \\
\hline $\mathrm{AC}$ & $\begin{array}{l}1 \mathrm{M} \mathrm{Et}_{4} \mathrm{NBF}_{4} \\
\text { in PC }\end{array}$ & $0-2.5$ & 2.1 & $\begin{array}{l}\text {-Capacitance quickly } \\
\text { drops at frequencies } \\
\text { more than } 10 \mathrm{mHz} \\
\text {-poor frequency } \\
\text { response }\end{array}$ & [54] \\
\hline $\mathrm{CDC}$ & $\begin{array}{l}1.5 \mathrm{M} \\
\mathrm{TEABF}_{4}\end{array}$ & $-1-1$ & $\begin{array}{l}180 \mathrm{Fcm}^{-3} \\
\text { (volumetric } \\
\text { capacitance of } \\
\text { single } \\
\text { electrode) }\end{array}$ & $\begin{array}{l}\text {-Capacitance drops to } \\
\text { half of its initial value } \\
\text { at scan rates as low as } \\
500 \mathrm{mVs}^{-1} \text {-poor rate } \\
\text { capability }\end{array}$ & [55] \\
\hline CNT & $\begin{array}{l}\mathrm{BMIM} / \\
\mathrm{BF}_{4}\end{array}$ & $-0.5-0.5$ & 0.428 & N/A & [53] \\
\hline Gr & $\begin{array}{l}\mathrm{PVA} / \\
\mathrm{H}_{3} \mathrm{PO}_{4}\end{array}$ & $0-1$ & $<0.4$ & $\begin{array}{l}\text {-The device was tested } \\
\text { up to the scan rate of } \\
0.01 \mathrm{Vs}^{-1}\end{array}$ & [59] \\
\hline OLC & $\begin{array}{l}1 \mathrm{M} \mathrm{Et}_{4} \mathrm{NBF}_{4} \\
\text { in PC }\end{array}$ & $0-3$ & $\begin{array}{l}1.7 \text { at } \\
1 \mathrm{Vs}^{-1}\end{array}$ & $\begin{array}{l}\text {-RC time constant of } 26 \\
\text { ms indicating good } \\
\text { frequency response. }\end{array}$ & [56] \\
\hline $\mathrm{AC}$ & $\begin{array}{l}1 \mathrm{M} \mathrm{Et}_{4} \mathrm{NBF}_{4} \\
\text { in PC }\end{array}$ & $0-3$ & $\begin{array}{l}11.6 \text { at } 500 \\
\mathrm{mVs}^{-1}\end{array}$ & $\begin{array}{l}-\mathrm{RC} \text { time constant of } \\
700 \text { ms. } \\
\text {-Capacitance drops } \\
\text { with increase in scan } \\
\text { rate }\end{array}$ & [56] \\
\hline Gr & $\begin{array}{l}\mathrm{PVA} / \\
\mathrm{H}_{2} \mathrm{SO}_{4}\end{array}$ & $0-1$ & 7.6 & $\begin{array}{l}\text { Capacitance drops by } \\
\text { about } 75 \% \text { scan rate of } \\
4 \mathrm{Vs}^{-1}\end{array}$ & [60] \\
\hline Ppy & $\begin{array}{l}\mathrm{PVA} / \\
\mathrm{H}_{3} \mathrm{PO}_{4}\end{array}$ & $-0.25-0.25$ & - & $\begin{array}{l}\text { Poor capacitive } \\
\text { performance at high } \\
\text { scan rate }\end{array}$ & [52] \\
\hline $\mathrm{MnO}_{2}$ & $1 \mathrm{M} \mathrm{Na} 2 \mathrm{SO} 4$ & $-0.2-1$ & $\begin{array}{l}338 \mathrm{Fg}^{-1} \text { at } 50 \\
\mathrm{mVs}^{-1}\end{array}$ & $\begin{array}{l}\text { The capacitance } \\
\text { quickly drops at higher } \\
\text { scan rates }\end{array}$ & [58] \\
\hline
\end{tabular}


There are a few reports in the literature about micro-supercapacitors based on metal oxide pseudo-capacitive materials. Xue et al. [58] reported micro-supercapacitors fabricated through micro-fluidic etching of films of manganese oxide nanofiber arrays. The device showed a specific capacitance of about $338 \mathrm{Fg}^{-1}$ at a very low discharge rate of $0.5 \mathrm{mAcm}^{-2}$, but it showed very high resistance at higher discharge rates. Microsupercapacitors with interdigital electrodes of hydrous $\mathrm{RuO}_{2}$ are reported by Liu et al. [61], which showed a specific capacitance of $10.5 \mathrm{mFcm}^{-2}$ at a $50 \mathrm{mVs}^{-1}$ scan rate. However, the capacitance quickly dropped to $3.1 \mathrm{mFcm}^{-2}$ at a higher scan rate of 500 $\mathrm{mVs}^{-1}$. The characteristics and properties of some of the reported micro-supercapacitors with interdigital electrodes are summarized in table 2.2.

\subsubsection{Micro-supercapacitors with 3D Electrodes}

Although there are several reports on fabrication of 3D electrodes for microbatteries in the literature $[44,62]$, there are very few reports about on-chip microsupercapacitors with 3D electrode design. Sun et al. [63] reported on-chip 3D electrodes for supercapacitorswhich were fabricated by electrodeposition of Ppy on 3D structure of nickel current collectors. The nickel current collectors were fabricated by an electroforming method on a pre-etched $\mathrm{SiO}_{2} / \mathrm{Si} 3 \mathrm{D}$ substrate. Later, Sun et al. improved their design and fabricated a full micro-supercapacitor cell [64]. They reported a specific capacitance of about $30 \mathrm{mFcm}^{-2}$ for a single electrode of their fabricated device.

Recently, Shen et al. [65] reported an on-chip micro-supercapacitor with 3D electrodes of activated carbon. The device was fabricated by injection of mixture of $\mathrm{AC}$ and a polymeric binder in pre-fabricated channel on a silicon chip which served as template. The channels were fabricated by etching silicon wafer by inductively coupled plasma 
(ICP) and after injection of active materials into the channels, the silicon walls between the electrodes were removed by a second ICP step. The electrode had a 3D interdigital design and the thickness of the electrodes was between 50 to $70 \mu \mathrm{m}$. The fabricated device showed a specific capacitance of about $90.7 \mathrm{mFcm}^{-2}$ and power density of about $5105 \mathrm{mWcm}^{-2}[65]$.

\subsection{Carbon-Microelectromechanical Systems (C-MEMS)}

C-MEMS refers to a manufacturing technique in which a pre-patterned photoresist structure is converted into carbon structure by heat treatment in vacuum or inert environment at temperatures around $1000{ }^{\circ} \mathrm{C}$ [66]. The photoresist is usually patterned with conventional lithography methods and both positive and negative tone photoresists can be used to fabricate carbon structures with various aspect ratios. The electrical resistivity of pyrolyzed photoresist carbon has been studied by Park et al. [67]. If negative photoresists such as SU-8 are utilized, it is possible to fabricate high aspect ratio carbon structure through C-MEMS technique. The SU-8 photoresist is a negativetone photoresist and is one of the most popular deep UV photoresists. This photoresist has very high optical transparency which makes it ideal for imaging near vertical sidewalls in thick resist film [68].

The C-MEMS fabrication process has two main steps. The first step is photolithography of the photoresist to make the desired patterns. The next step is the carbonization or pyrolysis of the photoresist structure. The photolithography process usually involves spin coating, soft-bake, near UV exposure, post-bake, and development. Pyrolysis is done by heating the developed structure in oxygen-free atmospheres (such as $\mathrm{N}_{2}$, forming gas or vacuum) $[66,68,69]$. These fabrication steps are schematically 
depicted in figure 2.4. By controlling the fabrication steps, a variety of carbon microstructures can be fabricated by C-MEMS technique. Some of the typical C-MEMS structures are shown in figure 2.5. The C-MEMS microstructures have been successfully utilized for fabrication of miniaturized Li-ion batteries, fuel cells, and biofuel cells as well as biological devices such as dielectrophoresis and DNA sensor arrays [66].

(a) Spin-coating photoresist

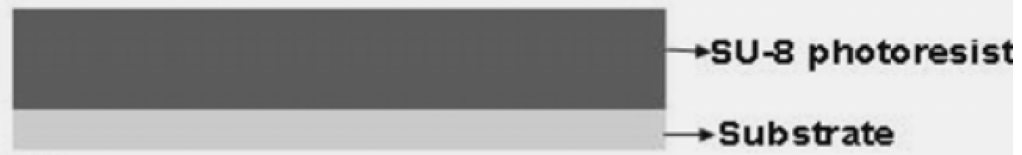

(b) UV exposure

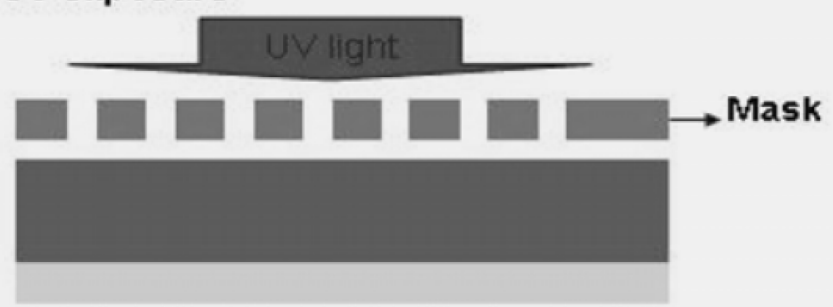

(c) Developing

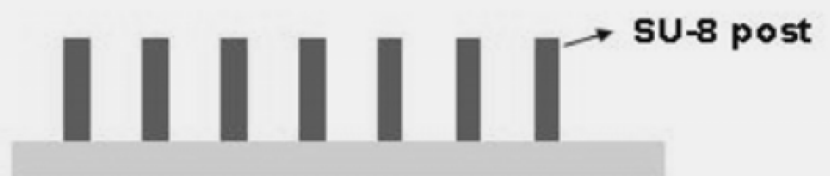

(d) pyrolysis

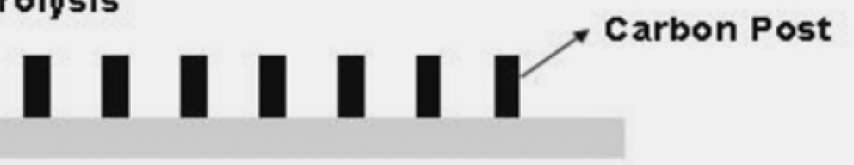

Figure 2.4 Process flow for fabrication of C-MEMS microstructures. Adapted from Ref. [69]. 

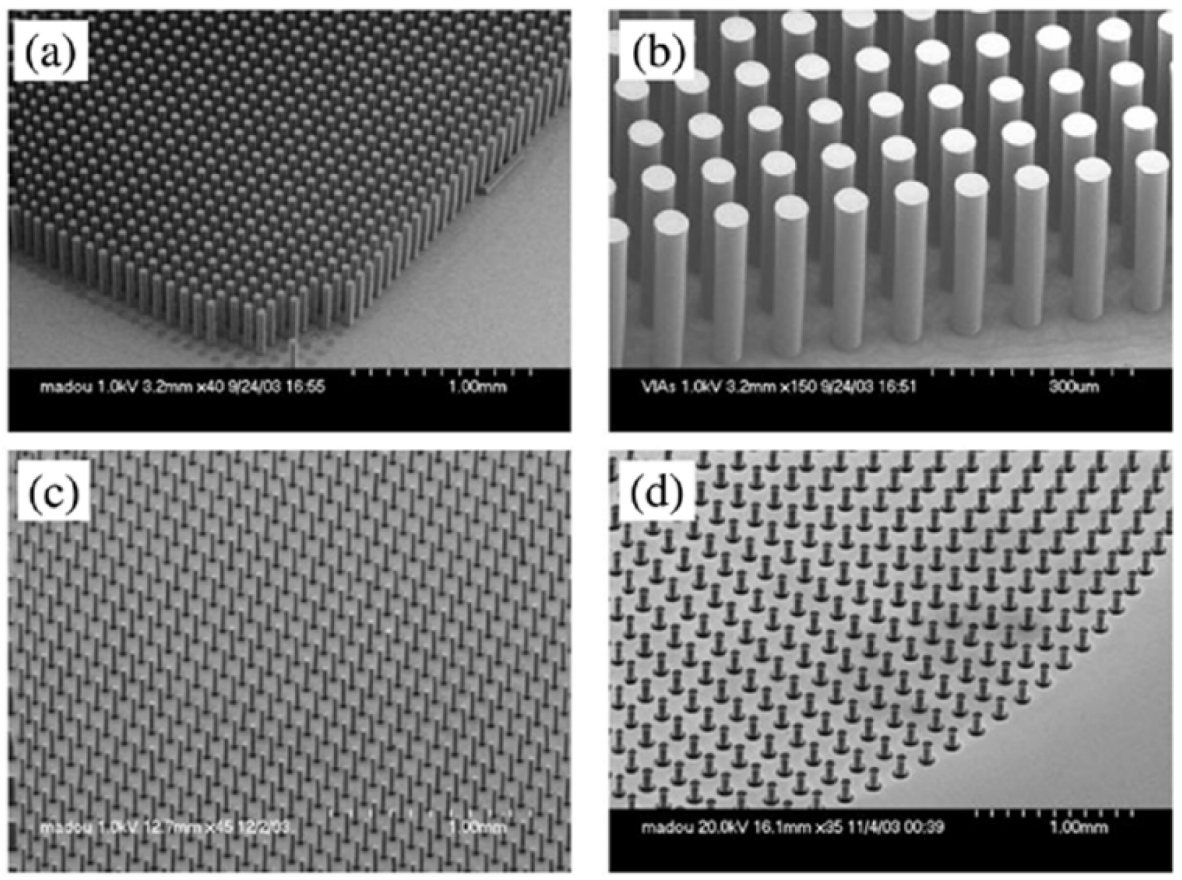

Figure 2.5 Typical SEM micrographs of SU-8 structures before pyrolysis (a, b), and carbon structures after pyrolysis of SU-8 (c, d). Adapted from Ref. [70].

\subsection{References}

[1] P. Simon, Y. Gogotsi, Nat. Mater. 7 (2008) 845-54.

[2] M. Armand, J.-M. Tarascon, Nature 451 (2008) 652-7.

[3] G. Wang, L. Zhang, J. Zhang, Chem. Soc. Rev. 41 (2012) 797-828.

[4] R. Kotz, M. Carlen, Electrochimica Acta 45 (2000) 2483-2498.

[5] Basic Research Needs for Electrical Energy Storage: Report of the Basic Energy Sciences Workshop on Electrical Energy Storage; April 2-4, 2007. Office of Basic Energy Sciences, DOE, July 2007.

[6] Becker HI. US Patent 2800616, 23 July 1957.

[7] B.E. Conway, Electrochemical Supercapacitors: Scientific Fundamentals and Technological Applications, Kluwer Academic/Plenum Publishers, New York, 1997.

[8] A. Pandolfo, A. Hollenkamp, J. Power Sources 157 (2006) 11-27. 
[9] M. Inagaki, H. Konno, O. Tanaike, J. of Power Sources 195 (2010) 7880-7903.

[10] P. J. Hall, M. Mirzaeian, S.I. Fletcher, F.B. Sillars, A.J.R. Rennie, G.O. ShittaBey, G. Wilson, A. Cruden, R. Carter, Energy \& Environ. Sci. 3 (2010) 1238.

[11] P. Lu, D. Xue, H. Yang, Y. Liu, International Journal of Smart and Nano Materials (2012) 1-25.

[12] D.N. Futaba, K. Hata, T. Yamada, T. Hiraoka, Y. Hayamizu, Y. Kakudate, O. Tanaike, H. Hatori, M. Yumura, S. Iijima, Nat. Mater. 5 (2006) 987-94.

[13] L. L. Zhang, X.S. Zhao, Chem. Soc. Rev. 38 (2009) 2520-31.

[14] E. Frackowiak, Phys. Chem. Chem. Phys. 9 (2007) 1774-85.

[15] O. Barbieri, M. Hahn, a Herzog, R. Kotz, Carbon 43 (2005) 1303-1310.

[16] K. Kierzek, Electrochim. Acta 49 (2004) 515-523.

[17] M. Endo, T. Maeda, T. Takeda, Y.J. Kim, K. Koshiba, H. Hara, M.S. Dresselhaus, J. of Electrochem. Soc. 148 (2001) A910.

[18] D. Qu, H. Shi, J. Power Sources 74 (1998) 99-107.

[19] X.S. Zhao, F. Su, Q. Yan, W. Guo, X.Y. Bao, L. Lv, Z. Zhou, J. Mater. Chem. 16 (2006) 637.

[20] M. Sevilla, S. Alvarez, T. Centeno, a Fuertes, F. Stoeckli, Electrochimica Acta 52 (2007) 3207-3215.

[21] Y. C. Choi, S.M. Lee, D.C. Chung, (2001) 497-500.

[22] J. S. Ye, H.F. Cui, X. Liu, T.M. Lim, W.-D. Zhang, F.-S. Sheu, Small 1 (2005) 560-5.

[23] Q. Xiao, X. Zhou, Electrochim. Acta 48 (2003) 575-580.

[24] K. H. An, W.S. Kim, Y.S. Park, J.-mi Moon, D.J. Bae, S.C. Lim, Y.S. Lee, Y.H. Lee, Adv. Funct. Mater. 5 (2001) 387-392.

[25] C. Du, N. Pan, Nanotechnology 17 (2006) 5314-5318.

[26] V. Presser, M. Heon, Y. Gogotsi, Adv. Funct. Mater. 21 (2011) 810-833.

[27] W. Deng, X. Ji, Q. Chen, C.E. Banks, RSC Advances 1 (2011) 1171. 
[28] J. H. Jang, A. Kato, K. Machida, K. Naoi, J. Electrochem. Soc. 153 (2006) A321.

[29] K. Naoi and P. Simon, Electrochem. Soc. Interf. 17 (2008) 34-37.

[30] A. Malak, K. Fic, G. Lota, C. Vix-Guterl, E. Frackowiak, J. Solid State Electrochem. 14 (2009) 811-816.

[31] V. Khomenko, E. Raymundo-Piñero, F. Béguin, J. Power Sources 153 (2006) 183190.

[32] J. Li, F. Gao, J. Power Sources 194 (2009) 1184-1193.

[33] K. A. Cook-Chennault, N. Thambi, A.M. Sastry, Smart Mater. Struct. 17 (2008) 043001.

[34] F. Albano, Y. Lin, D. Blaauw, D. Sylvester, K. Wise, a Sastry, Journal of Power Sources 185 (2008) 1524-1532.

[35] S. Tanaka, T. Yamada, S. Sugimoto, J. Micromech.Microeng. 13 (2003) 502-508.

[36] L.W. Weiss, Microsyst. Technolo. 15 (2008) 485-492.

[37] J. D. Morse, Int. J. Energy Res. 31 (2007) 576-602.

[38] H. A. Sodano, G.E. Simmers, R. Dereux, D.J. Inman, J. Intel. Mater. Syst. Struct. 18 (2006) 3-10.

[39] F. Herrault, C.-hyeon Ji, M.G. Allen, S. Member, J. MEMS 17 (2008) 1376-1387.

[40] W. M. Yang, S.K. Chou, C. Shu, Z.W. Li, H. Xue, Appl. Phys. Lett. 84 (2004) 3864.

[41] S. Roundy, P.K. Wright, Smart Mater. Struct. 13 (2004) 1131-1142.

[42] G. J. L. O, H.J. In, E. Crumlin, G. Barbastathis, Int. J. Energy Res. 31 (2007) 548575.

[43] J. A. Paradiso, Pervasive Comput. 4, 18-27 (2005).

[44] J. W. Long, B. Dunn, D.R. Rolison, H.S. White, Chem. Rev. 104 (2004) 4463-92.

[45] J. Chmiola, C. Largeot, P.L. Taberna, P. Simon, Y. Gogotsi, Science 328 (2010) $480-483$. 
[46] T. S. Arthur, D. J. Bates, N. Cirigliano, D. C. Johnson, P. Malati, J. M. Mosby, E. Perre, M. T. Rawls, A. L. Prieto, B. Dunn, MRS Bulletin 36 (2011) 523-531.

[47] J. H. Lim, D. J. Choi, H.-K. Kim, W.I. Cho, Y. S. Yoon, J. Electrochem. Soc. 148 (2001) A275.

[48] H. K. Kim, T. -Y. Seong, J. -H. Lim, W. Ii, Y. Soo, J. Power Sources 102 (2001) 167-171.

[49] A. White, R. C. Slade, Synth. Metals 139 (2003) 123-131.

[50] M. Kaempgen, C.K. Chan, J. Ma, Y. Cui, G. Gruner, Nano Lett. 9 (2009) 1872-6.

[51] L. Hu, J. W. Choi, Y. Yang, S. Jeong, F. La Mantia, L. -F. Cui, Y. Cui, PNAS 106 (2009) 21490-4.

[52] J. H. Sung, S. J. Kim, S. -H. Jeong, E. -H. Kim, K. -H. Lee, J. Power Sources 162 (2006) 1467-1470.

[53] Y. Q. Jiang, Q. Zhou, L. Lin, Proc. IEEE MEMS Conference, 587-590, Sorrento, 2009.

[54] D. Pech, M. Brunet, P.-L. Taberna, P. Simon, N. Fabre, F. Mesnilgrente, V. Conédéra, H. Durou, J. Power Sources 195 (2010) 1266-1269.

[55] M. Heon, S. Lofland, J. Applegate, R. Nolte, E. Cortes, J.D. Hettinger, P.-L. Taberna, P. Simon, P. Huang, M. Brunet, Y. Gogotsi, Energy Environ. Sci. 4 (2011) 135.

[56] D. Pech, M. Brunet, H. Durou, P. Huang, V. Mochalin, Y. Gogotsi, P. -L. Taberna, P. Simon, Nat. Nanotechnol. 5 (2010) 651-654.

[57] W. Gao, N. Singh, L. Song, Z. Liu, A. L. M. Reddy, L. Ci, R. Vajtai, Q. Zhang, B. Wei, P. M. Ajayan, Nat. Nanotechnol. 6 (2011) 6-10.

[58] M. Xue, Z. Xie, L. Zhang, X. Ma, X. Wu, Y. Guo, W. Song, Z. Li, T. Cao, Nanoscale 3 (2011) 2703-8.

[59] J. J. Yoo, K. Balakrishnan, J. Huang, V. Meunier, B. G. Sumpter, A. Srivastava, M. Conway, A. L. M. Reddy, J. Yu, R. Vajtai, P. M. Ajayan, Nano Lett. 11 (2011) 1423-7.

[60] Z. Weng, Y. Su, D. -W. Wang, F. Li, J. Du, H. -M. Cheng, Adv. Energy Mater. 1 (2011) 917-922 
[61] C. -C. Liu, D. -S. Tsai, D. Susanti, W. -C. Yeh, Y. -S. Huang, F. -J. Liu, Electrochim. Acta 55 (2010) 5768-5774.

[62] D. R. Rolison, J.W. Long, J.C. Lytle, A.E. Fischer, C.P. Rhodes, T.M. McEvoy, M.E. Bourg, A.M. Lubers, Chem. Soc. Rev. 38 (2009) 226-52.

[63] W. Sun, X. Chen, Microelec.Eng. 86 (2009) 1307-1310.

[64] W. Sun, X. Chen, J. Power Sources 193 (2009) 924-929.

[65] C. Shen, X. Wang, W. Zhang, F. Kang, J. Power Sources 196 (2011) 1046510471 .

[66] C. Wang, R. Zaouk, B. Y. Park, M. J. Madou, Int. J. Manufacturing Technology and Management, 13 (2008) 360-375.

[67] B. Y. Park, L. Taherabadi, C. Wang, J. Zoval, M. J. Madou, J. Electrochem. Soc. 152 (2005) J136.

[68] C. Wang, M. J. Madou, Biosensors and Bioelectronics 20 (2005) 2181-7.

[69] C. Wang, L. Taherabadi, G. Jia, M. J. Madou, Y. Yeh, B. Dunn, Electrochem. Solid State Lett. 7 (2004) A435.

[70] C. Wang, G. Jia, L.H. Taherabadi, M.J. Madou, J. MEMS 14 (2005) 348-358. 


\section{ELECTROCHEMICALLY ACTIVATED CARBON MICRO-ELECTRODE ARRAYS FOR ON-CHIP MICRO-SUPERCAPACIITOR APPLICATION}

\subsection{Introduction}

Development of miniaturized electronic systems has derived the demand for miniaturized power sources that can be integrated into such systems. Several kinds of micron-sized power sources such as micro-batteries [1], micro-fuel cells [2], and energy harvesters [3] have been developed in recent years. However, for the applications that require high power, there is a need for miniaturized electrochemical capacitors (microcapacitors) [4]. Electrochemical micro-capacitors with high power density can be coupled with energy harvesting devices to store the generated energy. Moreover, they can also be paired with micro-batteries to provide the peak power and improve the cycle lifetime. Based on the charge storage mechanism, electrochemical capacitors (ECs) can be divided into electric double layer capacitors (EDLCs) and pseudo-capacitors [5]. The former utilizes interfacial double layer capacitance of various types of carbon materials to store electric charge. The latter, the pseudo-capacitor or redox capacitor, uses fast and reversible surface or near-surface redox reactions for charge storage. The active materials of pseudo-capacititors include transition metal oxides and conductive polymers [5-6]. Micro-capacitors of both types have been reported in the literature [4, 7-10]. For example, Lim et al. [4] reported that a thin film EC based on pseudo-capacitive ruthenium oxide $\left(\mathrm{RuO}_{2}\right)$ and Lipon solid electrolyte delivered a volumetric capacitance of about $38 \mathrm{mFcm}^{-2} \mu \mathrm{m}^{-1}$, however its capacitance dropped by $53 \%$ after 500 cycles. Electrochemical micro-capacitor based on conductive polymer was first reported by Sung et al., who fabricated Polypyrrole (Ppy) micro-electrodes by electrochemical deposition 
on interdigitated gold electrodes [7]. More recently, Sun et al. [8] reported the fabrication of three dimensional (3D) PPy electrode architectures for micro-capacitors with geometric capacitance of the $27 \mathrm{mFcm}^{-2}$ (normalized by the footprint area) at $1 \mathrm{mAcm}^{-2}$ current density. On the other hand, EDLCs usually have higher rate capability, higher power density, and an extended cyclic life compared to pseudo-capacitors [6]. In recent years, there have been some efforts to fabricate micro-scale EDLCs $[9,10]$. For example, fabrication of printable thin film ECs with single-walled carbon nanotubes as electroactive materials has been reported by Kaempgen et al., where the estimated capacitance of the fabricated cell was $1.1 \mathrm{mFcm}^{-2}$, in a potential window of 0 to $1.0 \mathrm{~V}$ [9]. In addition, ECs from inkjet printing of activated carbon powders on interdigitated gold current collectors reached the maximum cell capacitance of $2.1 \mathrm{mFcm}^{-2}$ at a low scan rate of $1 \mathrm{mVs}^{-1}[10]$.

In this study, the carbon electrochemical systems (C-MEMS) technique was used to fabricate micro-electrodes for electrochemical capacitors. Furthermore, for the first time, an activation method was employed to improve electrochemical properties of CMEMS electrodes. The C-MEMS technique is a simple and reproducible process for fabricating various glassy carbon structures with micro patterns, in which patterned photoresist is pyrolyzed and converted into carbon under high temperatures in an inert atmosphere [11-13]. The advantages of using C-MEMS to fabricate micro-capacitor electrodes are that this technique is compatible with other MEMS processes and allows the possibility to fabricate $3 \mathrm{D}$ glassy carbon micro-scaled architectures. This technique has been used before by Wang et al. $[14,15]$ to fabricate carbon electrodes for microbatteries. Typical glassy carbon consists of embedded closed pores, which can be opened 
by an appropriate activation process, such as thermal activation and electrochemical activation $[16,17,18]$. In this study, the capacitive properties of as-prepared C-MEMS micro-electrode arrays were investigated. To improve the performance of the C-MEMS structures, electrochemical activation was employed. Cyclic voltammetry (CV) and galvanostatic charge-discharge experiments were conducted to evaluate electrochemical performance of the activated micro-electrodes. The capacitive performances of activated and non-activated micro-electrode arrays were compared.

\subsection{Experimental}

\subsubsection{Preparation of C-MEMS Electrodes}

The C-MEMS electrodes used in this study were prepared by a two-step photolithography process followed by a pyrolysis step. The substrate was $\mathrm{SiO}_{2}(2000$ $\AA$ ) $/ \mathrm{Si}$ and two kinds of negative tone photoresists, NANO ${ }^{\mathrm{TM}}$ SU-8 25 and SU-8 100 (MicroChem Corp.), were used for the lithography process. In each step, development was carried out using a $\mathrm{NANO}^{\mathrm{TM}}$ SU-8 developer (MicroChem Corp.). All other chemicals were purchased from Sigma-Aldrich. UV lithography was performed using an OAI 800 mask aligner. Detailed fabrication steps of C-MEMS structures are schematically presented in Figure 3.1a. In brief, a two dimensional interdigitated finger pattern was firstly created using the photolithography of SU-8 25 photoresist. SU-8 25 was spin coated on the substrate with initial speed of $500 \mathrm{rpm}$ and then accelerated to $3000 \mathrm{rpm}$ and stayed for $30 \mathrm{~s}$. Then, the spin coated photoresist was baked for $3 \mathrm{~min}$ at $65^{\circ} \mathrm{C}$ and $7 \mathrm{~min}$ at $95^{\circ} \mathrm{C}$ on a leveled hotplate. The baked photoresist was patterned with a

UV exposure dose of $300 \mathrm{mJcm}^{-2}$. Post-exposure bake was done for $1 \mathrm{~min}$ at $65^{\circ} \mathrm{C}$ and 5 min at $95^{\circ} \mathrm{C}$ on a leveled hotplate. Next, another photolithography process was employed 
using the SU-8 100 photoresist to create cylindrical posts on patterned fingers. In this step of the process, SU-8 100 was first spin coated on a finger patterned substrate, where a speed of $500 \mathrm{rpm}$ was chosen to spread the photoresist, after which the speed was increased to $1500 \mathrm{rpm}$ and kept at this speed for $30 \mathrm{~s}$. Spin coated photoresist was then baked for $10 \mathrm{~min}$ at $65^{\circ} \mathrm{C}$ and $45 \mathrm{~min}$ at $95^{\circ} \mathrm{C}$ in an oven. The exposure was done using a UV exposure dose of $700 \mathrm{mJcm}^{-2}$. Post-exposure bake was performed for $3 \mathrm{~min}$ at $65^{\circ} \mathrm{C}$ and $10 \mathrm{~min}$ at $95^{\circ} \mathrm{C}$ in an oven. Then the sample was developed in the SU-8 developer. Finally, the resulting SU-8 structures were pyrolyzed at $1000{ }^{\circ} \mathrm{C}$ for $1 \mathrm{~h}$ in forming gas atmosphere (i.e., $95 \%$ nitrogen and 5\% hydrogen). To remove the residual carbon between the fingers after pyrolysis, all samples were subjected to oxygen plasma treatment at 400 mTorr with a power of $150 \mathrm{~W}$ for $20 \mathrm{~s}$ prior to other experimental investigations. After plasma treatment electrical resistance between the interdigitated electrodes was measured and the resistance in the order of mega Ohms was confirmed. The total numbers of samples which are used for different characterization methods were 28 interdigitated C-MEMS micro-electrode arrays (Schematically shown in Figure 1b).

\subsubsection{Electrochemical Activation}

To perform electrochemical activation on both electrodes of each C-MEMS micro-capacitor, the two electrodes were connected together through the contact pads (schematically shown in Figure 3.1b) with a piece of silver wire. Then the contact pads and the silver wire were fully covered by epoxy resin to prevent their exposure to the electrolyte. Subsequently, the sample was composed into a three-electrode system as the working electrode. The reference and the counter electrodes were $\mathrm{Ag} / \mathrm{AgCl}$ and a Pt wire, respectively. Electrochemical activation was performed in $0.5 \mathrm{M} \mathrm{H}_{2} \mathrm{SO}_{4}$ solution 
deaerated by nitrogen bubbling. A voltage of $1.9 \mathrm{~V}$ was applied to the electrodes for the durations of either 10 or 30 min using a multichannel potentiostat/galvanostat (VMP3,
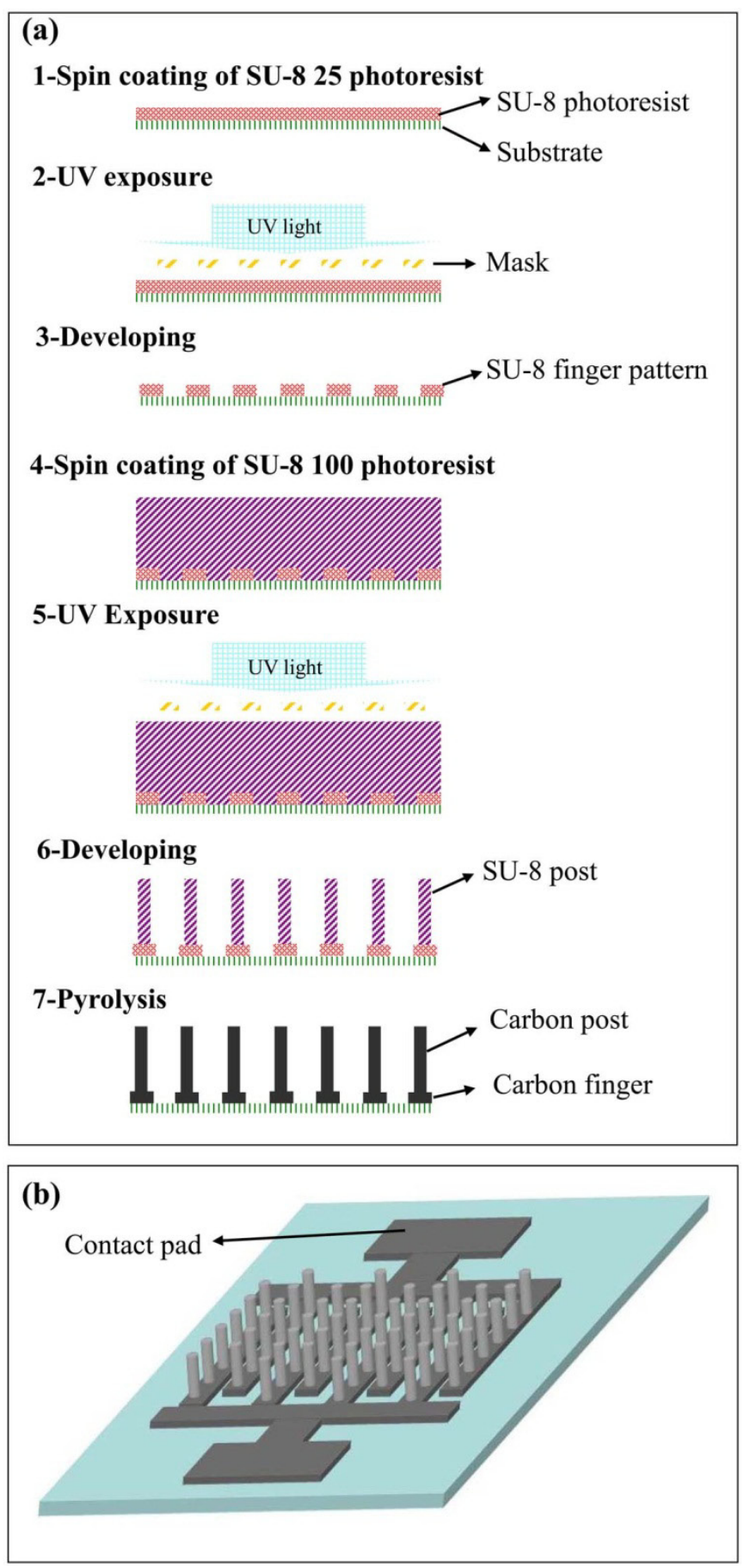

Figure 3.1 (a) Schematic of a typical process flow for fabricating C-MEMS electrodes. (b) Schematic 3D view of a sample after carbonization. 
Princeton Applied Research). The electrodes were then negatively polarized at $-0.3 \mathrm{~V}$ for 10 min. After electrochemical pretreatment, the electrodes were washed with DI water.

\subsubsection{Characterization}

The microstructure of electrodes was investigated by a JOEL 6335 field-emission scanning electron microscope (FE-SEM). Electrochemical performance tests were performed in two-electrode electrochemical cells, where one of the interdigitated electrodes acted as the working electrode and the other as the counter electrode. The electrolyte was deaerated $0.5 \mathrm{M} \mathrm{H}_{2} \mathrm{SO}_{4}$ aqueous solution. CVs were recorded at different scan rates ranging from 5 to $200 \mathrm{mV} \mathrm{s}^{-1}$. Galvanostatic charge/discharge experiments were performed under various current densities in the voltage range between 0 and $1 \mathrm{~V}$. All the electrochemical tests were carried out in room temperature.

For each sample, geometric surface area and volume were measured using SEM and optical microscope images. The density of the photoresist-derived carbon was measured by calculating the weight of 5 carbon films with known thickness and geometric area. The average density was $1.52 \mathrm{~g} \mathrm{~cm}^{-3}$. The weight of each electrode was estimated by multiplying geometric volume into the average density of photoresist derived carbon. To confirm the accuracy of measured weights by this method, weights of 5 electrodes were measured by subtracting the measured weight before and after the removal of the electrodes from the substrate. The measured weights by the two methods were in good agreement and the average deviation in calculated weights was about $5 \%$.

In this work, three different terms were used to describe the corresponding capacitance of the electrodes. For each electrode, specific gravimetric capacitance, 
specific geometric capacitance and specific volumetric capacitance is the capacitance of the electrode normalized by its mass, geometric surface area and volume, respectively.

\subsection{Results and Discussion}

\subsubsection{Structural Characterizations}

Figure 3.2 shows typical SEM micrographs of C-MEMS electrodes. Carbon posts are perfectly aligned on carbon fingers and the device has two interdigitated 3D electrodes.

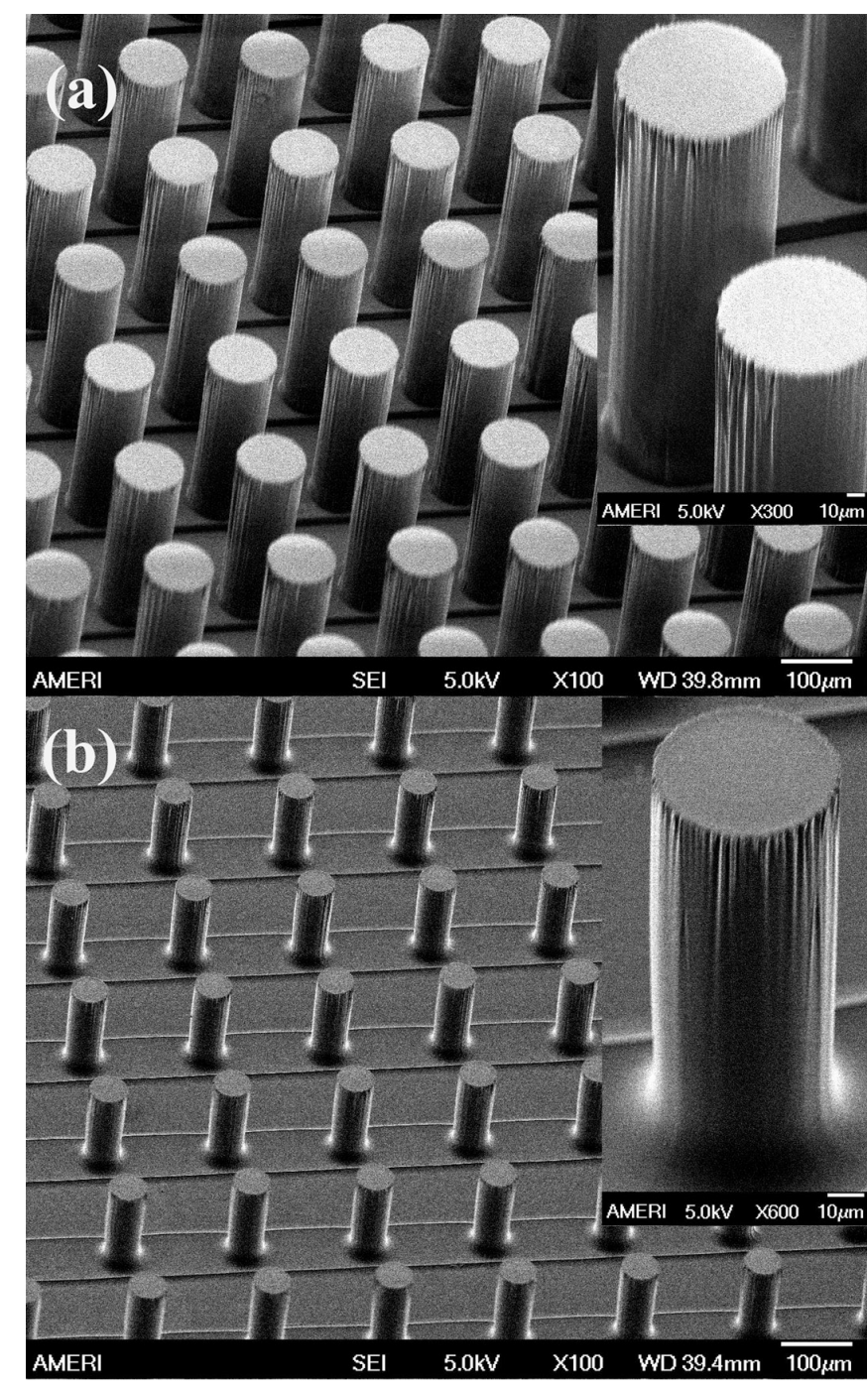

Figure 3.2 SEM images of C-MEMS electrodes. (a) SU-8 structure (b) carbonized structure. The insets show higher magnification images. 
The total footprint area of a typical sample is $9 \mathrm{~mm} \times 9 \mathrm{~mm}$ with a total of 50 interdigitated fingers ( 25 fingers for each electrode) and the finger widths were $\sim 100 \mu \mathrm{m}$. After carbonization, the measured post diameters of samples ranged from about 53 to 68 $\mu \mathrm{m}$. The heights of carbon posts of different samples varied from about 115 to $140 \mu \mathrm{m}$ with an average of $\sim 130 \mu \mathrm{m}$. The origin of variation of post height and diameter for different samples is attributed to the difference in wafer chip size and small variations of the amount of photoresist used for spin coating during fabrication of each sample. We predict that the dimensions of carbon post for different samples can be effectively controlled by optimizing experimental parameters during the fabricating process. Due to the good adhesion of SU-8 25 to the substrate, the shrinkage of fingers during carbonization is less than the posts. On the other hand, the adhesion of SU-8 fingers and posts are also very good. Therefore, the shrinkage of structure in contact regions of fingers and posts are controlled by shrinkage of fingers. As a result, the posts have shrunk less near the base of the structures than at the midsection [14].

\subsubsection{Electrochemical Tests}

\section{$\underline{\text { Non-activated samples }}$}

$\mathrm{CV}$ was used to determine electrochemical properties of non-activated samples. Figure 3.3a shows the CV curves of a non-activated sample at various scan rates. The CV curves show a near rectangular shape at all scan rates. The average current is low, ranging from 3.48 to $2.84 \mu \mathrm{A}$, and increases by increasing the scan rate. The capacitance of the two electrode cells can be calculated according to the following equation [19, 20]:

$$
C=\frac{\int I(V) d V}{2 s \Delta V}
$$


where $\int I(v) d V$ is the total voltammetric charge obtained by the integration of positive and negative sweep in cyclic voltammograms, $s$ is the scan rate and $\Delta V$ is the width of the potential window.
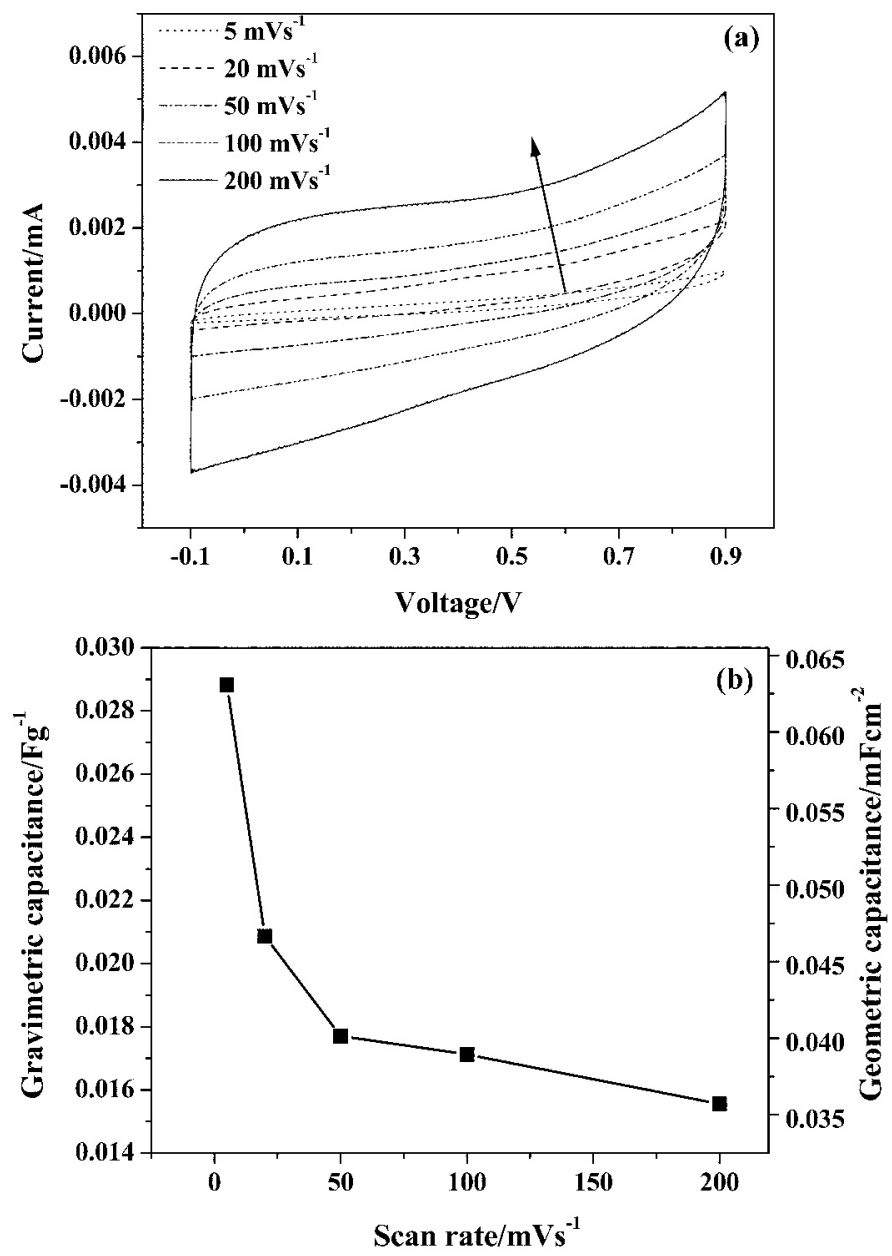

Figure 3.3 (a) CVs of a non-activated sample (the arrow shows the direction of increasing scan rate). (b) The specific gravimetric and geometric capacitance of a non-activated sample at various scan rates.

The capacitance calculated from Eq. (3.1) is the total capacitance of the cell which is the sum of the two equivalent single electrode capacitors in the series. To calculate the specific gravimetric and geometric capacitance of each electrode, equations Eq. (3.2) and (3.3) have been used respectively. 
$C=\frac{2 C}{m}$

$C=\frac{2 C}{A}$

where $C_{m}$ is the specific gravimetric capacitance, $\mathrm{C}$ is the total capacitance of the cell, $\mathrm{m}$ is the mass, $C_{A}$ is the specific geometric capacitance and $\mathrm{A}$ is the total geometric surface area of a single electrode. The two electrodes of the device have identical design specifications and have been fabricated simultaneously during the fabrication process. Moreover, the electrodes were activated at the same time in one electrochemical cell. Therefore, for calculating the capacitance, we have assumed that the two electrodes of the device have identical capacitance value.

Figure $3.3 \mathrm{~b}$ shows the specific gravimetric and geometric capacitances of CMEMS electrodes measured at different scan rates of 5, 20, 50, 100, and $200 \mathrm{mVs}^{-1}$. The specific capacitance decreases gradually by increasing the scan rate and the highest value of geometric capacitance of $\sim 0.07 \mathrm{mFcm}^{-2}$ is found at the lowest scan rate of $5 \mathrm{mVs}^{-1}$. At this scan rate the specific gravimetric capacitance is calculated as $\sim 0.03 \mathrm{~F} \mathrm{~g}^{-1}$. Considering the relatively smooth and pore-free surface of the photoresist derived carbon structures, the low specific capacitance of as-pyrolysed C-MEMS electrodes is reasonable [21]. This implies the active surface of these electrodes is limited to their measurable geometric surface.

\section{Electrochemically activated samples}

Figure 3.4a presents the $\mathrm{CV}$ curves at different scan rates of a sample activated for $30 \mathrm{~min}$. The curves at all scan rates show a near rectangular shape and a broad band was observed on top of these rectangular responses. It is known that the anodic oxidation 
of samples increases the amount of oxygen groups on the electrode surface [17]. A broad peak at $0.2 \sim 0.4 \mathrm{~V}$ during anodic sweep can be attributed to the contribution of active oxygen surface groups generated by electrochemical treatment [22]. CV studies of electrochemically activated glassy carbon by Nagaoka et al. also show a similar broad peak on anodic curves [23]. In addition, the shapes of anodic/cathodic sweeps are asymmetric, which is in accordance with the reports by Sullivan et al. [17, 22] and Nagaoka et al. [23]. This is due to either slow electron transfer between surface redoxactive groups and the bulk glassy carbon, or charging effects resulting from small pores and microcracks $[17,22,23]$.

In Figure $3.4 \mathrm{~b} C \mathrm{C}$ curves of a non-activated and a sample activated for $30 \mathrm{~min}$ are compared at the same $100 \mathrm{mVs}^{-1}$ scan rate. The area of the activated sample's CV curve is significantly larger than that of the non-activated sample, which implies the enhancement of the specific capacitance after activation. The gravimetric and geometric specific capacitance of samples activated for $30 \mathrm{~min}$ and $10 \mathrm{~min}$ were calculated from $\mathrm{CV}$ curves (shown in Figure 3.4c). For all samples, the specific capacitance decreases with an increase in the scan rate. The specific gravimetric capacitance of the sample activated for $30 \min \left(\sim 33 \mathrm{Fg}^{-1}\right)$ was almost three orders of magnitude higher than the non-activated sample $\left(\sim 0.03 \mathrm{Fg}^{-1}\right)$. At a scan rate of $5 \mathrm{mVs}^{-1}$, the geometric capacitance for the nonactivated and activated (30 min) samples was $\sim 0.07 \mathrm{mFcm}^{-2}$ and $\sim 75 \mathrm{mFcm}^{-2}$.

The geometric capacitance of the sample activated for 10 min was $\sim 22 \mathrm{mFcm}^{-2}$. At the same scan rate, the volumetric capacitance of samples activated for 10 and $30 \mathrm{~min}$ was $\sim 14 \mathrm{Fcm}^{-3}$ and $\sim 48 \mathrm{Fcm}^{-3}$, respectively. 

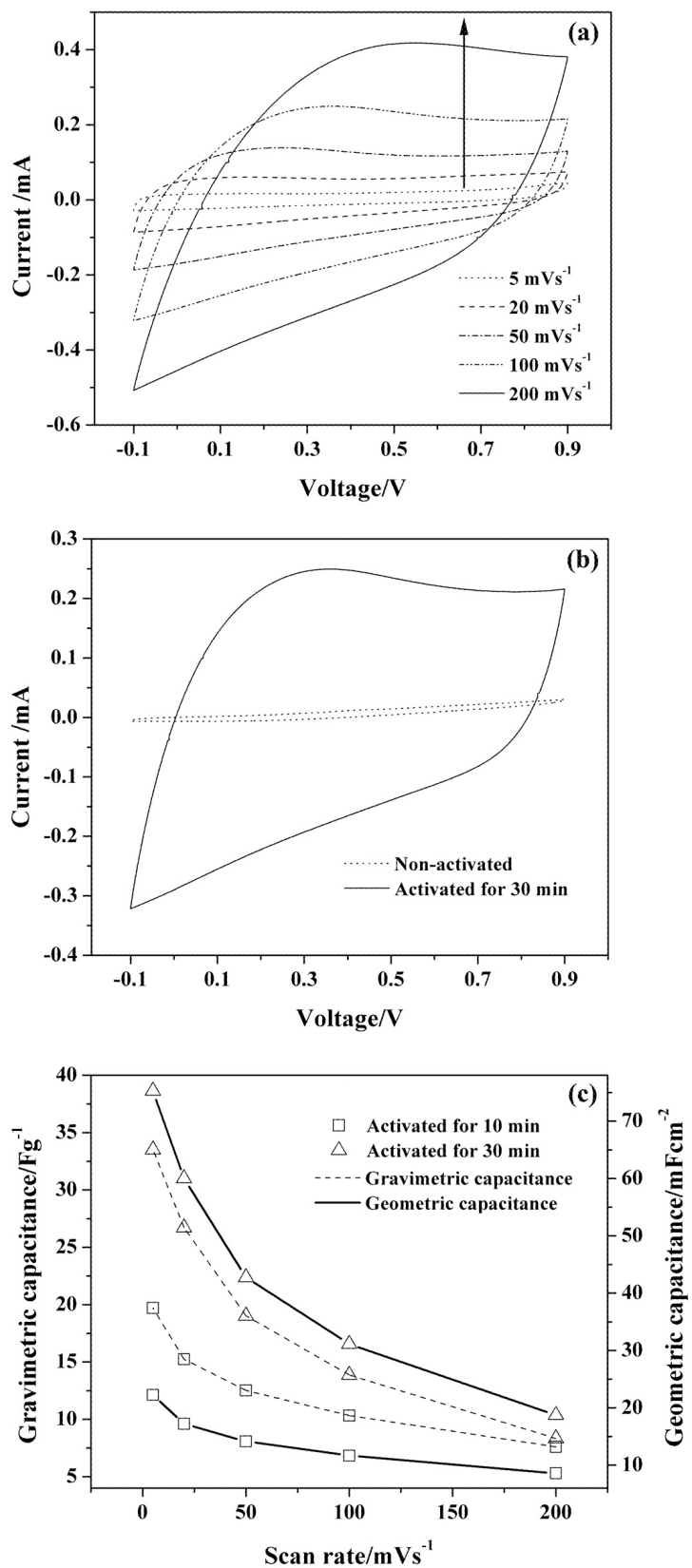

Figure 3.4 (a) CVs of microelectrodes in two-electrode cells after activation for $30 \mathrm{~min}$ at different scan rates (the arrow shows the direction of increasing scan rate). (b) Typical CVs of samples before and after activation for 30 minutes in $0.5 \mathrm{M} \mathrm{H}_{2} \mathrm{SO}_{4}$ aqueous electrolyte at 100 $\mathrm{mVs}^{-1}$ scan rate. (c) The specific gravimetric and geometric capacitance of activated samples at various scan rates. 


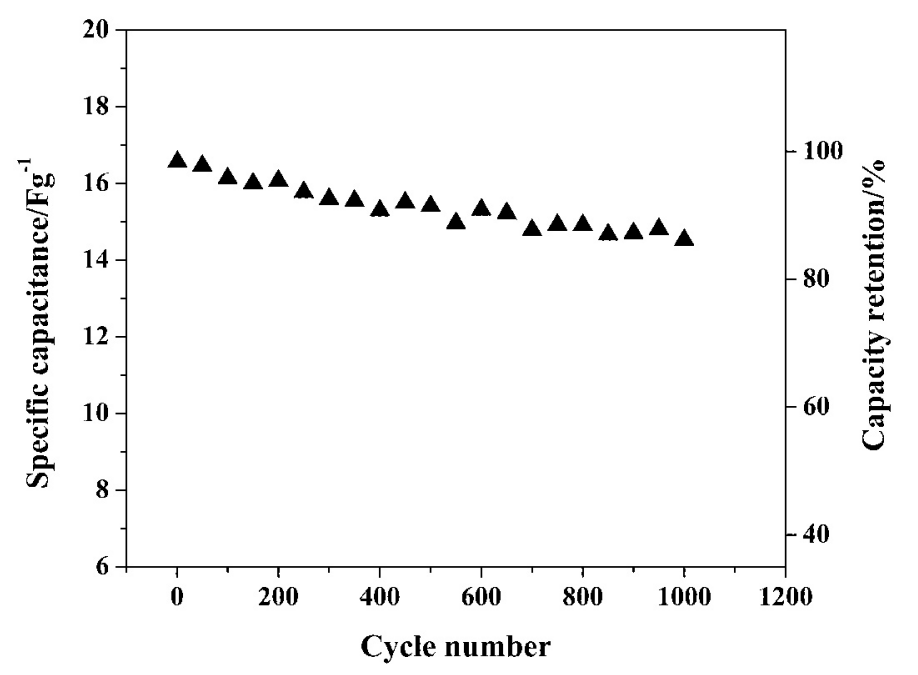

Figure 3.5 Cyclic performance and capacity retention for an activated sample.

The specific capacitances at different scan rates of the sample activated for 30 min are higher than that of samples activated for $10 \mathrm{~min}$ at corresponding scan rates. The C-MEMS sample activated for 30 min was also examined by CV tests for 1000 cycles at the scan rate of $50 \mathrm{mVs}^{-1}$ in the two electrode system. The capacitance value has been plotted and shown in Figure 3.5. The specific capacitance of this sample at the first cycle was $\sim 17 \mathrm{~F} \mathrm{~g}^{-1}$. After 1000 cycles, the specific capacitance was $15 \mathrm{~F} \mathrm{~g}^{-1}$ and there was only $12.3 \%$ fade of the initial capacitance. This indicates acceptable cyclability of activated micro-electrodes compared to electrochemical micro-capacitors reported by Lim et al., where $53 \%$ of the initial capacitance faded after 500 cycles [4].

In order to further investigate the performance of electrochemically activated CMEMS micro-electrodes, galvanostatic charge/discharge experiments were performed on activated samples with various current densities in a voltage range of 0-1.0 V. Examples of charge/discharge curves of the samples activated for $30 \mathrm{~min}$ at various discharge current densities are shown in Figure 3.6a. The charging half of each charge/discharge 
cycles is almost a straight line if excluding the initial $i R$ drop. The discharge curves also have small $i R$ drops at the beginning as shown in Figure 3.6a. After the $i R$ drop, the decrease of voltage with increasing time deviates from a straight line. Deviation from ideality in voltage-time curves has also been observed for electrochemically modified graphite electrodes by Xu et al. [24] Niu et al. [25] also reported non-linearity in voltagetime curves of porous C-cloth material. While pseudo-capacitive contribution from redox-active surface groups can cause the deviation from linearity in charge/discharge curves, Niu et al. [25] explained that other factors may also contribute to this non-ideal behavior. These factors include redistribution of charge within the pores of activated electrodes during charging or discharging and the effect of direct equivalent series resistance (ESR). The discharge capacitance of the cell was measured from the discharge curves after the $i R$ drop, according to the following equation [26]:

$C=I \times \Delta t \times \Delta V^{-1}$

where $I$ is the current and $\Delta t$ is the time interval for the change in voltage range $\Delta V$. The specific gravimetric and geometric capacitance of the electrodes was calculated using Eq. (3.2) and Eq. (3.3), respectively.

Figure $3.6 \mathrm{~b}$ shows the variation of the specific gravimetric and geometric capacitance of activated samples at various discharge rates. The geometric capacitance is $\sim 48 \mathrm{mFcm}^{-2}$ at $1 \mathrm{mAcm}^{-2}$. Specific gravimetric capacitance decreases from $\sim 24 \mathrm{Fg}^{-1}$ at 0.5 mAcm ${ }^{-2}$ discharge current density to $\sim 11 \mathrm{Fg}^{-1}$ at $5 \mathrm{mAcm}^{-2}$ discharge current density. These results are in agreement with the above CV results. The improvements in electrochemical performance of activated electrodes can be explained by the following reasons. 

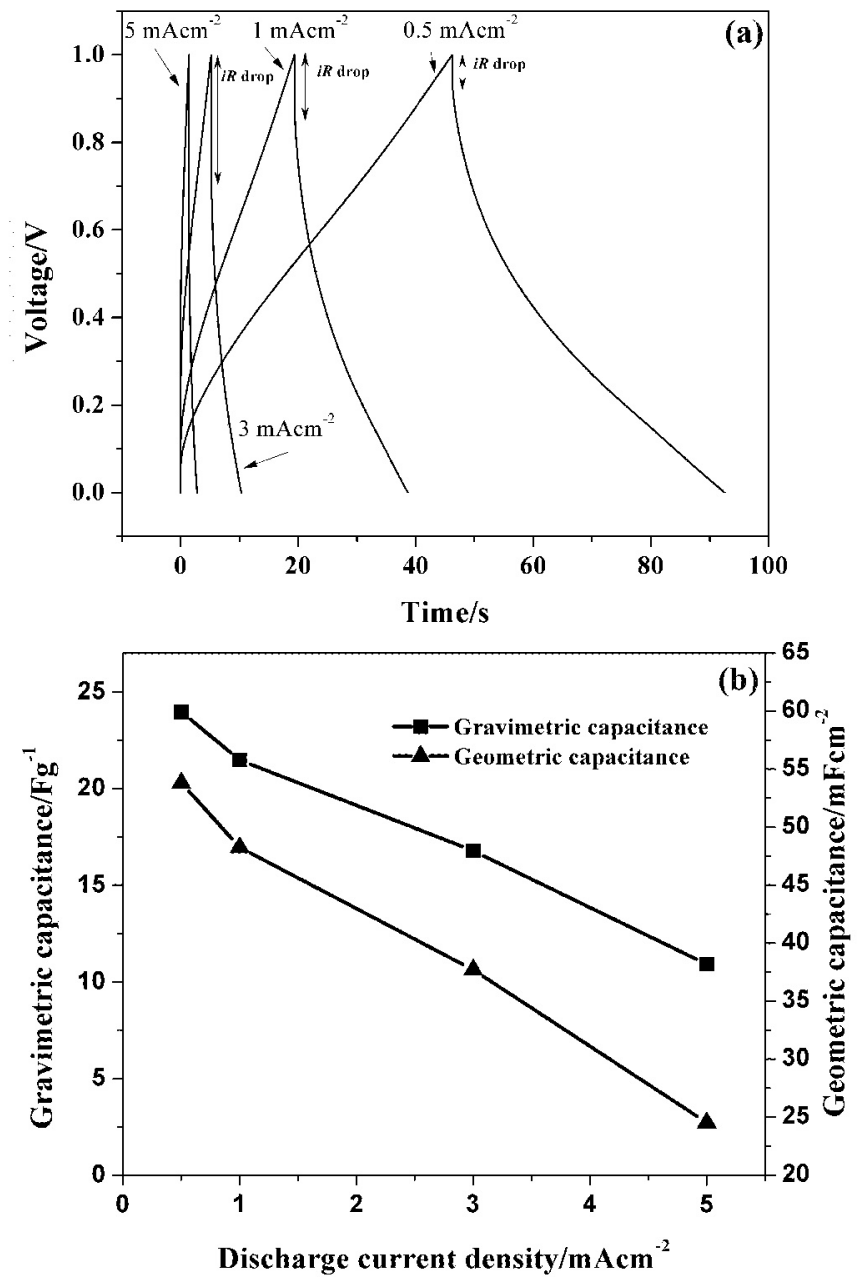

Figure 3.6 (a) Galvanostatic charge/discharge curves at various discharge rates. (b) The specific gravimetric and geometric capacitance of activated samples at various discharge rates. The sample was electrochemically activated for $30 \mathrm{~min}$.

Firstly, introducing oxygen groups to the surface carbon electrodes is able to improve the wettability of the electrode surface and contribute additional pseudocapacitance [27]. While the appearance of a current maximum on CV curves (Figure 3.4a) proves the existence of oxygen groups on the surface of the electrodes, further studies need to be done to quantitatively evaluate their contribution to the capacitive performance of the electrodes. Secondly, the increase in the surface area of the electrodes 
after activation seems to be the main reason of enhancement of specific capacitance since the electrochemical pretreatment oxidizes the carbon surface and thus open up the internal closed pores, which is demonstrated by Sullivan et al. [22]. Braun et al. [28] had suggested that this activation process creates a large and electrochemically accessible internal surface area, which thus increases the double layer capacitance. Our attempts to analyze the pore size of the electrodes by studying gas adsorption isotherms could not deliver reliable results mainly because the small amount of active materials for each micro fabricated sample makes it impossible to have a precise sorption measurement. Further investigations need to be done in order to quantify the increase in surface area of the electrodes after electrochemical activation.

Another possible reason for the increase in the capacitance of the electrodes after electrochemical treatment is the irreversible intercalation of ions during polarization. This phenomenon has been observed for graphitizable carbon [29]. It is reported that, during the first polarization cycle ions are inserted into the carbon structure. This intercalation process is irreversible and provides a larger specific capacitance in successive cycles [29]. To investigate intercalation in C-MEMS electrodes, we performed polarization on electrodes for several cycles (between 0 to $-2.5 \mathrm{~V}$ ). No significant irreversible increase in the capacitance was observed for the C-MEMS electrodes.

Compared to previously published carbon based electrochemical micro-capacitor works $[9,10]$, our micro-capacitors based on C-MEMS shows excellent and promising performance. Despite the significant improvement after electrochemical activation, the gravimetric capacitance of the C-MEMS electrode is still lower than that of activated carbon powders with very high BET surface area which is widely used in ECs. 
It was reported that $\mathrm{KOH}$ activated carbon with a BET surface area of $3150 \mathrm{~m}^{2} \mathrm{~g}^{-1}$ shows specific gravimetric capacitance of $312 \mathrm{Fg}^{-1}$ in a $1 \mathrm{M} \mathrm{H}_{2} \mathrm{SO}_{4}$ solution [30]. This can be reasonable considering the activation mechanism during electrochemical activation. As mentioned above, electrochemical activation forms an active porous film on the surface of the electrode. Braun et al. [28] showed that the formation of the active film begins at the outer surface of the carbon and extends into the interior gradually during the activation process, surrounding an inactive core with closed pores. The thickness of the active layer linearly increases with an increase in the activation time as reported by Sullivan et al. [22]. For the activation time of $30 \mathrm{~min}$ used in this study, we can expect the thickness of the active film to be just a few microns. This indicates that a carbon layer with thickness of only a few microns on the surface of carbon posts contribute to the capacitance of electrodes and a large amount of the mass of the electrode is not accessible to the electrolyte. It is assumed that by increasing the surface area and decreasing the mass of electrodes through fabrication of high aspect ratio carbon posts, the gravimetric specific capacitance can be improved. Furthermore, as projected from Figure 3.4c electrochemical activation of electrodes for longer durations could result in a further increase in the specific capacitance of samples. Moreover, based on the proposed model of development of porous structures on the surface of glassy carbon electrodes by Sullivan et al. [22] and Braun et al. [16], we can assume that at the initial stages of activation, the pore entries are too small for the electrolyte to penetrate. As activation advances, pore walls would be thinned, and thus pores will grow in size. Therefore, increasing the activation time will also enhance the accessibility of the pores, which in turn enhances the electrochemical capacitance of the electrodes under high 
charge-discharge current conditions. Enhancement of the accessibility of the electrolyte will also improve the non-ideal behavior which was observed in the voltage-time curve. Our attempts to further increase the activation time is not repeatable since the carbon current collectors (fingers) delaminated from the surface of the wafer in the case of longer activation times. Further research is underway to improve the adhesion of carbon fingers to the substrate.

The present study demonstrates that C-MEMS fabricated interdigitated microelectrodes are potentially capable of delivering energy storage solutions for microdevices. Compared to as-prepared electrodes, electrochemical activation of electrodes for 30 min increased the capacity by three orders of magnitude. Fabrication of higher aspect ratio micro-electrodes can increase the surface area of the device in the limited footprint area thus increasing the capacitance normalized by the footprint of the device. Future developments will include fabrication of higher aspect ratio 3D electrodes, increasing the adhesion of carbon current collectors to the substrate, and optimizing the conditions of electrochemical activation.

\subsection{Conclusion}

The C-MEMS technique was used to fabricate interdigitated micro-electrode arrays for on-chip electrochemical micro-capacitors. Electrochemical characterization of electrochemically activated micro-electrodes shows excellent capacitive behavior. Specific geometric capacitance of $\sim 75 \mathrm{mFcm}^{-2}$ was achieved after electrochemical activation for $30 \mathrm{~min}$. For this activation duration the volumetric capacitance was $\sim 48$

$\mathrm{Fcm}^{-3}$. These results indicate that the C-MEMS technique is a very promising method for the fabrication of electrochemical micro-capacitors. However, the non-ideal voltage-time 
curves and rapid drop in capacitance at high scan rates suggests that activation conditions should be improved to enhance the electrochemical properties of micro-devices.

\subsection{References}

[1] F. Albano, Y.S. Lin, D. Blaauw, D.M. Sylvester, K.D. Wise, A.M. Sastry, J. Power Sources 185 (2008) 1524-1532.

[2] J.D. Morse, Int. J. Energy Res. 31 (2007) 576-602.

[3] K. A. Cook-Chennault, N. Thambi, A. M. Sastry, Smart Mater. Struct. 17 (2008) 043001.

[4] J.H. Lim, D.J. Choi, H.K. Kim, W.I. Cho, Y.S. Yoon, J. Electrochem. Soc. 148 (2001) A275-A278.

[5] B. E. Conway, W. G. Pell, J. Solid State Electrochem. 7 (2003) 637-644.

[6] P. Simon, Y. Gogotsi, Nat. Mater. 7 (2008) 845-854.

[7] J.-W. Sung, S.-J. Kim, K.-H. Lee, J. Power Sources 133 (2004) 312-319.

[8] W. Sun, X. Chen, J. Power Sources 193 (2009) 924-929.

[9] M. Kaempgen, C.K. Chan, J. Ma, Y. Cui, G. Gruner, Nano. Lett. 9 (2009) 18721876.

[10] D. Pech, M. Brunet, P.-L.Taberna, P. Simon, N. Fabre, F. Mesnilgrente, V. Conédéra, H. Durou, , J. Power Sources 195 (2010) 1266-1269.

[11] C.Wang, R. Zaouk, B. Y. Park, M. J. Madou, Int. J. Manufacturing Technology and Management, 13 (2008) 360-375.

[12] C. Wang, G. Jia, L. H. Taherabadi, M. J. Madou, J. Microelectromech. Syst. 14 (2005) 348-358.

[13] H. Xu, K. Malladi, C. Wang, L. Kulinsky, M. Song, M. J. Madou, Biosens. Bioelectron. 23 (2008) 1637-1644.

[14] C. Wang, L. Taherabadi, G. Jia, M. Madou, Y. Yeh, B. Dunn, Electrochem. Solid State Lett. 7 (2004) A435-A438.

[15] J. W. Long, B. Dunn, D. R. Rolison, H. S. White, Chem. Rev. 104 (2004) 44634492. 
[16] A. Braun, M. Bärtsch, O. Merlo, B. Schnyder, B. Schaffner, R. Kötz,O. Haas , A. Wokaun, Carbon 41 (2003) 759-765.

[17] M.G. Sullivan, B. Schnyder, M. Bärtsch, D. Alliata, C. Barbero, R. Imhof, R. Kötz, J. Electrochem. Soc. 147 (2000) 2636-2643.

[18] A. Dekanski, J. Stevanovic, R. Stevanocic, B. Z. Nikolic, V. M. Jovanovic, Carbon 39 (2001) $1195-1205$.

[19] H. Li, J. Wang, Q. Chu, Z. Wang, F. Zhang, S. Wang, J. Power Sources 190 (2009) 578-586.

[20] B. E. Conway, Electrochemical Supercapacitors: Scientific Fundamentals and Technological Applications, Kluwer, New York, 1999.

[21] A. Singh, J. Jayaram, M. Madou, S. Akbar, J. Electrochem. Soc., 149 (3) (2002) E78-E83.

[22] M. G. Sullivan, R. Kötz, O. Haas, J. Electrochem. Soc. 147 (2000) 308-317.

[23] T. Nagaoka, T.Yoshino, Anal. Chem. 58 (1986) 1037-1042.

[24] H. Xu, X. Fan, Y. Lu, L. Zhong, X. Kong, J. Wang, Carbon 48 (2010 ) 3293-3311.

[25] J. Niu, W. G. Pell, B. E. Conway, J. Power Sources 156 (2006) 725-740.

[26] J. Wang, Y. Xu, X. Chen, X. Du, J. Power Sources 163 (2007) 1120-1125.

[27] E. Frackowiak, Phys. Chem. Chem. Phys. 9 (2007) 1774-1785.

[28] A. Braun, J. Kohlbrecher, M. Bärtsch, B. Schnyder, R. Kötz, O. Haas , A. Wokaun, Electrochim. Acta 49 (2004) 1105-1112.

[29] P.W. Ruch, M. Hahn, D. Cericola, A. Menzel, R. Kötz, A. Wokaun, Carbon 48 (2010) 1880-1888.

[30] K. Kierzek, E. Frackowiak, G. Lota, G. Gryglewicz, J. Machnikowski, Electrochim. Acta 49 (2004) 515-523. 


\section{MICRO-SUPERCAPACITORS BASED ON THREE DIMENSIONAL INTERDIGITAL POLYPYRROLE/C-MEMS ELECTRODES}

\subsection{Introduction}

Supercapacitors are fast charging energy storage devices with moderate energy density, which have higher power densities as compared to batteries and higher energy densities when compared to conventional capacitors. Based on the energy storage mechanism, supercapacitors are of two types: (a) electric double layer capacitors (EDLCs) which electrostatically store charge on the electrode/electrolyte interface of high surface area materials, and (b) pseudo-capacitors or redox capacitors which store charge in the bulk of a redox material in response to fast surface and near-surface redox reactions $[1,2]$. When used in conjunction with batteries, supercapacitors improve the performance by expanding the cycle life and providing the peak power when it is needed. The recent development in small and portable electronic devices has increased the demand for power sources that are sufficiently compact and are potentially able to be integrated on a chip with other electronic components. Miniaturized electrochemical capacitors or micro-supercapacitors are expected to be the power sources for miniaturized electronic devices [3-7].

Most of the recent research efforts in the micro-supercapacitor field have focused on increasing energy and power densities by improving the material properties and architecture of the devices. From the material aspect, various nanostructured materials have been utilized as electrode materials for micro-supercapacitors. Several carbonaceous nanomaterials such as carbon nanotubes (CNT) [4], activated carbons [6], carbide derived carbons [3], graphene [8], and carbon onions [5] have been used to fabricate EDLC 
micro-supercapacitors. Moreover, transition metal oxides such as Ruthenium oxide [9] and conductive polymers such as polypyrrole (PPy) [10] have been used as materials for pseudo-capacitive micro-supercapacitors. From the device architecture aspect, there are mainly two types of micro-supercapacitor architectures reported in the literature, one composed of thin film electrodes of nanostructured materials $[4,11]$ and the other consisting of small interdigital finger electrodes $[3,5-7,10]$. Recently, the latter type has gained more interest. This architecture offers two main advantages in comparison to conventional two dimensional (2D) stacking of thin film electrodes: (a) having both electrodes in the same plane (easier for on-chip integration) and (b) having an improved performance and kinetics compared to the thin film devices of the same materials [3]. The device performance could be further enhanced by employing three dimensional (3D) electrodes with high effective surface area. This has been verified by the improved performance of micro-batteries [12] and micro-supercapacitors [13] with 3D interdigital electrodes fabricated through micro-molding or deep reactive ion etching (DRIE). However, more facile fabrication techniques are needed to expand the applications of this type of micro-supercapacitor.

Previously, we reported fabrication of micro-supercapacitors through Carbonmicroelectrochemical systems (C-MEMS) technology $[7,14]$. The specific capacitance of as-prepared C-MEMS electrodes could be improved by electrochemical activation [7] and integration of CNTs on the electrodes [14]. In this study, we describe a different approach to enhance energy and power density of micro-supercapacitors by employing a combination of a high surface area C-MEMS current collector and a pseudo-capacitive material with high capacitance. PPy is a low cost and environmentally friendly capacitive 
material with high energy density and it has been the subject of numerous studies as the electrode material for supercapacitors [15]. In this research, PPy was electrochemically deposited on 3D C-MEMS structures to develop a pseudo-capacitive microsupercapacitor. Cyclic voltammetry (CV) and galvanostatic charge-discharge experiments were conducted to evaluate the electrochemical performance of both single PPy/C-MEMS electrodes and full symmetric cells. We demonstrated that the power and energy density of PPy based micro-supercapacitors can be improved by structuring the electrodes to 3D interdigital electrode architectures.

\subsection{Experimental}

\subsubsection{Design of the Electrodes}

We have designed and tested single micro-electrodes and symmetric microsupercapacitors of PPy on 3D interdigital current collectors which have been fabricated through the C-MEMS technique. Structuring the electrodes in a 3D platform offers higher effective surface area in footprint of devices compared to thin film and 2D interdigital electrodes. This fact has been schematically illustrated in Figure 4.1a. The ratio of effective surface area of the 3D electrode to the $2 \mathrm{D}$ electrode $\left(A_{\text {eff }}\right)$ can be calculated from Eq. (4.1):

$$
A_{e f f}=\frac{N \pi D H}{L W}+1
$$

where $\mathrm{L}$ and $\mathrm{W}$ are the length and width of the projected surface area (finger), D, H and $\mathrm{N}$ are the diameter, height and number of carbon posts, respectively. With the dimensions of the electrodes used in this study, i.e., $7000,80,50$, and 140 microns for $\mathrm{L}$, 
$\mathrm{W}, \mathrm{D}$, and $\mathrm{H}$, respectively, and by having 39 carbon posts on each finger $(\mathrm{N}=39)$ we can achieve $A_{e f f}=2.53$.

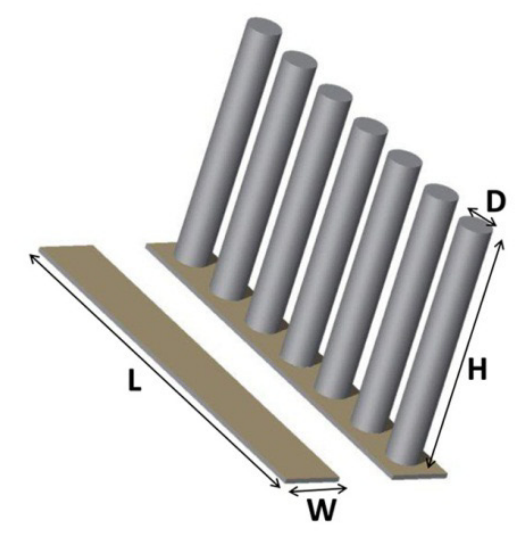

(a)

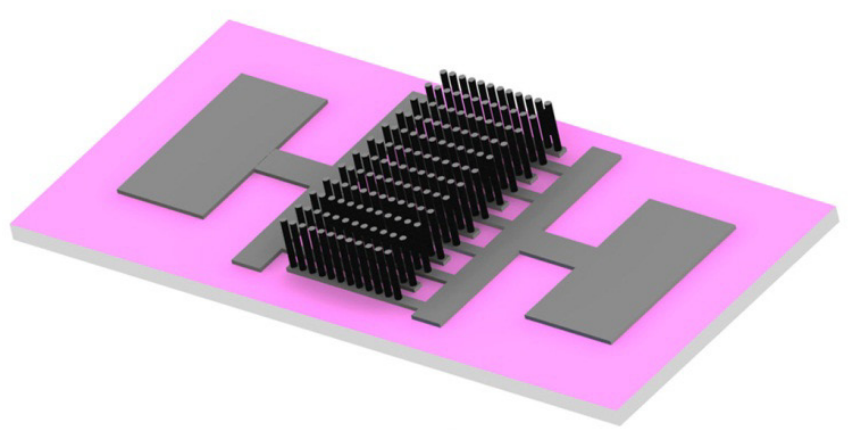

(b)

Figure 4.1 (a) 2D and 3D design of interdigital electrodes. (b) Schematic 3D view of a PPy/CMEMS micro-supercapacitor.

\subsubsection{Fabrication of C-MEMS Structures}

The Preparation of C-MEMS electrode has been described in detail elsewhere [7]. Briefly, a thin layer of negative tone photoresist (SU-8 25, MicroChem Corp.) was spin coated on a $\mathrm{SiO} 2 / \mathrm{Si}$ wafer. Subsequently the photoresist was baked for $3 \mathrm{~min}$ at $65^{\circ} \mathrm{C}$ and 7 min at $95^{\circ} \mathrm{C}$ on a leveled hotplate, and patterned with a UV exposure dose of 300 $\mathrm{mJcm}{ }^{-2}$. Post-exposure bake was done for $1 \mathrm{~min}$ at $65^{\circ} \mathrm{C}$ and $5 \mathrm{~min}$ at $95^{\circ} \mathrm{C}$ on a leveled 
hotplate. A second layer of photoresist with much higher thickness (SU-8 100, MicroChem Corp.) was spin coated on top of the patterned fingers. This layer was baked for $10 \mathrm{~min}$ at $65^{\circ} \mathrm{C}$ and $45 \mathrm{~min}$ at $95^{\circ} \mathrm{C}$ in an oven. The exposure was done using a UV exposure dose of $700 \mathrm{mJcm}^{-2}$. Post-exposure bake was performed for $3 \mathrm{~min}$ at $65^{\circ} \mathrm{C}$ and $10 \mathrm{~min}$ at $95^{\circ} \mathrm{C}$ in an oven. Then the sample was developed in the SU-8 developer. Finally, the resulting SU-8 structures were pyrolyzed at $1000^{\circ} \mathrm{C}$ for $1 \mathrm{~h}$ in a forming gas atmosphere (i.e., 95\% nitrogen and 5\% hydrogen). The residual carbon between the fingers was then removed by oxygen plasma etching at 400 mTorr with a power of 150 W for 20 s.

\subsubsection{Electropolymerization of PPy Films}

In order to electrochemically deposit PPy film on the C-MEMS current collectors, the two electrodes of the device were connected together and the device was set as a working electrode in an electrochemical cell. $\mathrm{An} \mathrm{Ag} / \mathrm{AgCl}$ electrode and a platinum wire served as a reference electrode and a counter electrode, respectively. PPy was electrochemically deposited on the C-MEMS current collectors from an electrolyte solution of $0.1 \mathrm{M}$ Pyrrole monomer and $0.5 \mathrm{M} \mathrm{LiClO}_{4}$ supporting salt at constant current density of $1 \mathrm{mAcm}^{-2}$ for durations of 5 to $15 \mathrm{~min}$. After the deposition, electrodes were washed with DI water and dried in air. Figure $4.1 \mathrm{~b}$ schematically shows the prepared PPy/C-MEMS micro-supercapacitor. For comparison, 2D film electrodes were also fabricated by electrodeposition of PPy on pyrolyzed photoresist carbon films which were prepared by carbonizing thin SU-8 photoresist films at the same condition as carbonizing 3D electrodes. The deposition of PPy was performed using the same deposition 
conditions and solution as deposition of PPy on 3D C-MEMS electrodes. The surface area of carbon film electrodes was about $0.5 \mathrm{~cm}^{2}$.

\subsubsection{Characterization}

The Microstructure of PPy/C-MEMS electrodes were studied by a field emission scanning electron microscope (FE-SEM, JEOL 7000F). The electrochemical performance of electrodes was tested using a multichannel potentiostat/galvanostat (VMP3, Princeton Applied Research) with both three-electrode and two-electrode systems in $0.1 \mathrm{M} \mathrm{KCl}$ aqueous electrolyte. In the three-electrode system a single PPy/C-MEMS electrode served as the working electrode, while a Pt wire and $\mathrm{Ag} / \mathrm{AgCl}$ electrode were the counter and reference electrodes, respectively. In the two-electrode system, one of the interdigital electrodes was the working electrode while the other electrode acted as both the counter and the reference electrode. The dimensions of C-MEMS current structures were estimated from SEM images. The density of deposited PPy was calculated from a PPy film deposited on a large carbon film substrate $\left(2.5 \mathrm{~cm}^{2}\right.$ surface area). First the weight of the PPy film was estimated by subtracting the weight of the electrode before PPy deposition from the weight of the electrode after electropolymerization of PPy and drying. Then, the thickness of the electrode was estimated from the cross-sectional SEM images and profilometry measurements. The estimated density of PPy film is about 1.48 $\mathrm{gcm}^{-3}$, which is very close to the reported density of PPy in the literature (i.e., $1.5 \mathrm{gcm}^{-}$

$\left.{ }^{3}\right)[16]$. For the electrodes with 5, 10 and 15 min electropolymerization time, the thickness of PPy films are $0.5 \pm 0.03,1.2 \pm 0.09$ and $2 \pm 0.08$ microns, respectively. 


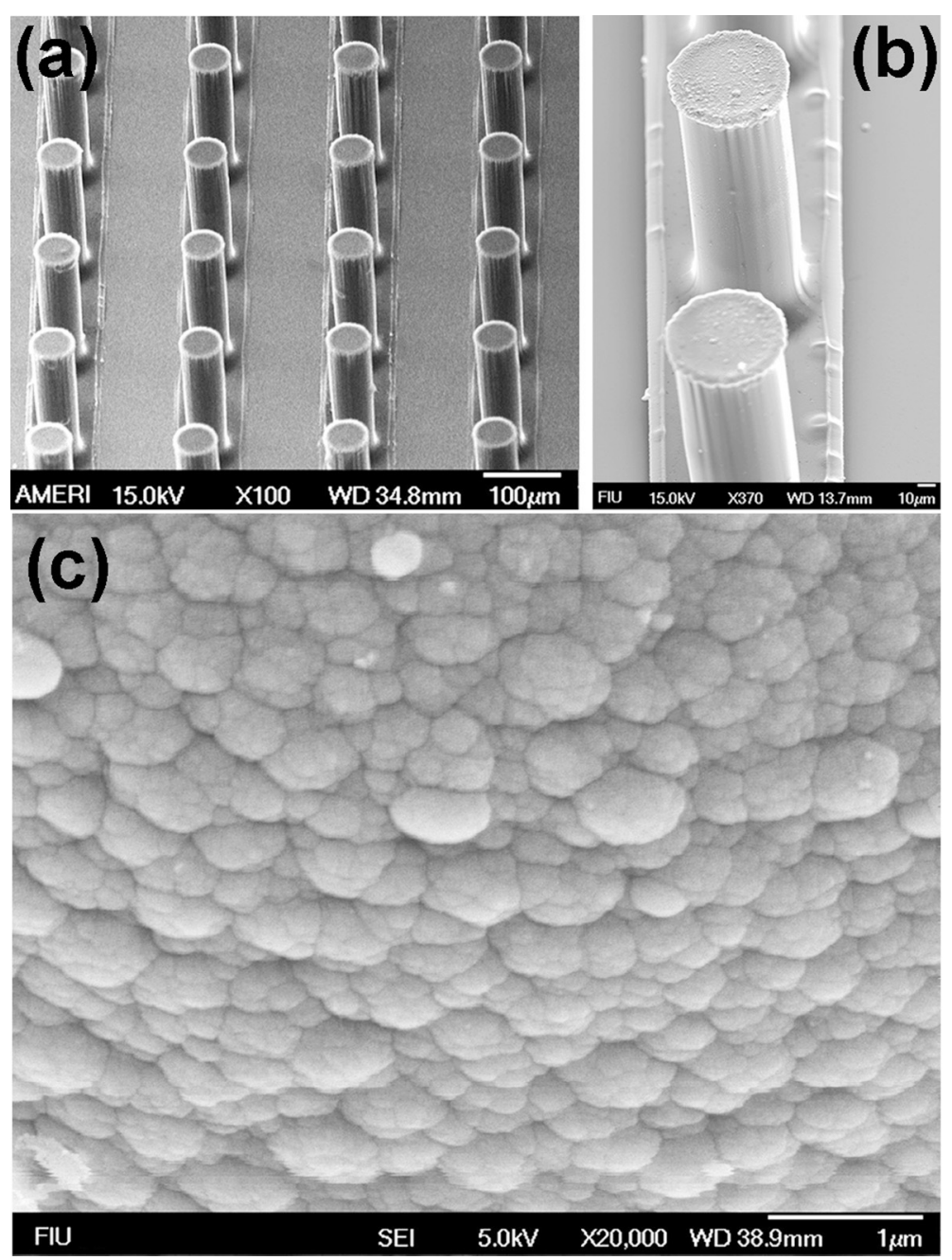

Figure 4.2 SEM images of (a) as-pyrolyzed C-MEMS electrodes (b) C-MEMS electrodes with PPy film. (c) High magnification image from the wall of a carbon post showing nanostructure of PPy film.

\subsection{Results and Discussion}

SEM micrographs of typical PPy/C-MEMS micro-supercapacitors are sown in Figure 4.2. These images show a conformal coating of PPy on the interdigital C-MEMS electrodes (both fingers and posts). Typical samples have a finger width of about $80 \mu \mathrm{m}$ and an average post diameter and height of 50 and $140 \mu \mathrm{m}$, respectively. Higher magnification image taken from the wall of a carbon post (Figure 4.2c) show a 
nanostructured granular raspberry-like morphology, which is typical of perchlorate-doped PPy [17].

Capacitive properties of materials can be revealed by Cyclic Voltammetry (CV). Voltammograms with a rectangular shape, high reaction current, and symmetric in anodic and cathodic directions, are an indication of ideal capacitive behavior. The performance of single electrodes and full cells were examined by $\mathrm{CV}$ at different scan rates ranging from 5 to $100 \mathrm{mVs}^{-1}$. Figure $4.3 \mathrm{a}$ shows the $\mathrm{CV}$ curves of single PPy/C-MEMS electrodes with PPy polymerization durations of 5, 10 and 15 min at a scan rate of 20 $\mathrm{mVs}^{-1}$ in $1 \mathrm{M} \mathrm{KCl}$ electrolyte. The $\mathrm{CV}$ response of single electrodes clearly shows the capacitive characteristics and small redox features of PPy under doping and de-doping of ions. All the $\mathrm{CV}$ curves exhibit a mirror image characteristic with respect to the potential axis at zero current and the reactive current density is higher for the electrodes with longer electrodeposition of PPy. The capacitance of single electrodes can be calculated by integrating the current of $\mathrm{CV}$ curves over the whole potential range according to Eq. (4.2):

$C=\frac{\int I(V) d V}{2 s \Delta V}$

where $\int I(V) d V$ is the total voltammetric charge obtained by the integration of positive and negative sweep in cyclic voltammograms, $s$ is the scan rate and $\Delta V$ is the width of the potential window. Figure $4.3 \mathrm{~b}$ shows the discharge curves of electrodes at $1 \mathrm{mAcm}^{-2}$ current density. The discharge curves are near straight lines after the initial $i R$ drop and discharge time increases with the increase in electropolymerization time of the electrodes. 
The discharge capacitance can be calculated from linear parts of discharge curves according to Eq. (4.3):

$C=I \times \Delta t \times \Delta V^{-1}$

where $I_{A}$ is the current and $\Delta t$ is the time interval for the change in voltage range $\Delta V$. Following the suggestion by Stoller et al. [18] for calculation of specific capacitance from galvanostatic discharge curve, $\Delta \mathrm{V}$ in Eq. (3) is from the middle of the voltage range to maximum voltage (Vmax).

Table 4.1 Specific capacitances of the electrodes with different polymerization time from CV (20 $\left.\mathrm{mVs}^{-1}\right)$ and galvanostatical discharge $\left(1 \mathrm{mAcm}^{-2}\right)$.

\begin{tabular}{ccccc}
\hline & \multicolumn{4}{l}{ Specific capacitance $\left(\mathrm{mFcm}^{-2}\right)$} \\
\cline { 2 - 5 } & \multicolumn{2}{l}{$\begin{array}{l}\text { Normalized by geometric surface } \\
\text { area }\end{array}$} & \multicolumn{3}{l}{ Normalized by footprint area } \\
\cline { 2 - 5 } \begin{tabular}{c} 
Test method \\
\cline { 2 - 5 } $\begin{array}{c}\text { Polymerization time } \\
(\text { min) }\end{array}$
\end{tabular} & $\mathrm{CV}$ & $\begin{array}{c}\text { Galvanostatic } \\
\text { discharge }\end{array}$ & $\begin{array}{c}\text { Galvanostatic } \\
\text { discharge }\end{array}$ \\
\hline 5 & $12.99 \pm 1.38$ & $11.98 \pm 1.61$ & $29.99 \pm 3.18$ & $27.64 \pm 3.72$ \\
10 & $48.70 \pm 7.89$ & $52.91 \pm 5.17$ & $112.39 \pm 11.28$ & $122.10 \pm 11.94$ \\
15 & $70.23 \pm 5.38$ & $82.60 \pm 7.55$ & $162.07 \pm 12.4$ & $190.61 \pm 17.47$ \\
\hline
\end{tabular}

The specific capacitance calculated from $\mathrm{CV}$ and discharge curves are presented in Table 4.1. Specific capacitance is calculated by normalizing the capacitance value by both geometric surface area (the surface area of carbon post and fingers) and footprint area of the electrodes (projected area). As shown in this table, both geometric area normalized and footprint area normalized capacitances are increasing with the increase in polymerization time. For the electrodes with a 15 min polymerization time, specific capacitances normalized by the geometric surface area are $70.23 \pm 5.38$ and $82.60 \pm 7.55$ $\mathrm{mFcm}^{-2}$ from $\mathrm{CV}$ and galvanostatic discharge curves, respectively. At this polymerization 
time, specific capacitances normalized by the footprint of the electrodes are $162.07 \pm 12.4$ and $190.61 \pm 17.47 \mathrm{mFcm}^{-2}$ from $\mathrm{CV}$ and discharge curves, respectively. The CV tests were performed on the single electrodes at various scan rates (Figure 4.4a). However, we chose to highlight the specific capacitance from the $\mathrm{CV}$ test at a $20 \mathrm{mVs}^{-1}$ scan rate in Table 2, since at this scan rate the discharge rate is similar to that of a typical supercapacitor application [18]. There are some discrepancies between the specific capacitance calculated from $\mathrm{CV}$ curves at $20 \mathrm{mVs}^{-1}$ and galvanostatic discharge curves at $1 \mathrm{mAcm}^{-2}$. The difference is more pronounced in the case of the electrode with the thickest PPy film (Polymerized for $15 \mathrm{~min}$ ). The main origin of these discrepancies is the difference between the discharge rates of the electrodes when tested with galvanostatic charge/discharge at $1 \mathrm{mAcm}^{-2}$ and $\mathrm{CV}$ at $20 \mathrm{mVs}^{-1}$. Discharge rates for galvanostatic charge/discharge tests can be calculated from Figure 4.3b and are about 100, 16, and 10 $\mathrm{mVs}^{-1}$ for the electrodes with PPy polymerization times of 5,10 and $15 \mathrm{~min}$, respectively. Comparison of calculated specific capacitance from the two methods should only be made at a similar discharge rate during the tests. In addition, for the electrodes with thicker PPy film the diffusion of the electrolyte ions to the lower levels of PPy film is limited. The diffusion of ions along the depth of film is more difficult at higher charge/discharge rates. In other words, at higher charge/discharge rates, ions cannot diffuse through the whole thickness of the PPy film in the time frame of capacitive measurement and only a portion of the film can contribute to the measured capacitance [19]. The weight of the PPy on the C-MEMS electrodes was estimated using the calculated data for surface area of the micro-electrodes and thickness and density of PPy films. The estimated specific gravimetric capacitances of the electrodes with 5, 10 and 15 
min electropolymerization time are $175.66 \pm 18.60,274.23 \pm 27.55$ and $237.27 \pm 18.19 \mathrm{Fg}^{-1}$, respectively. Although the electrodes with 15 min electropolymerization time have higher footprint area normalized capacitance as well as a higher specific geometric capacitance, they have a lower specific gravimetric capacitance. This again shows that due to the limited diffusion of ions in thick PPy films a portion of the material is not utilized during the tests.
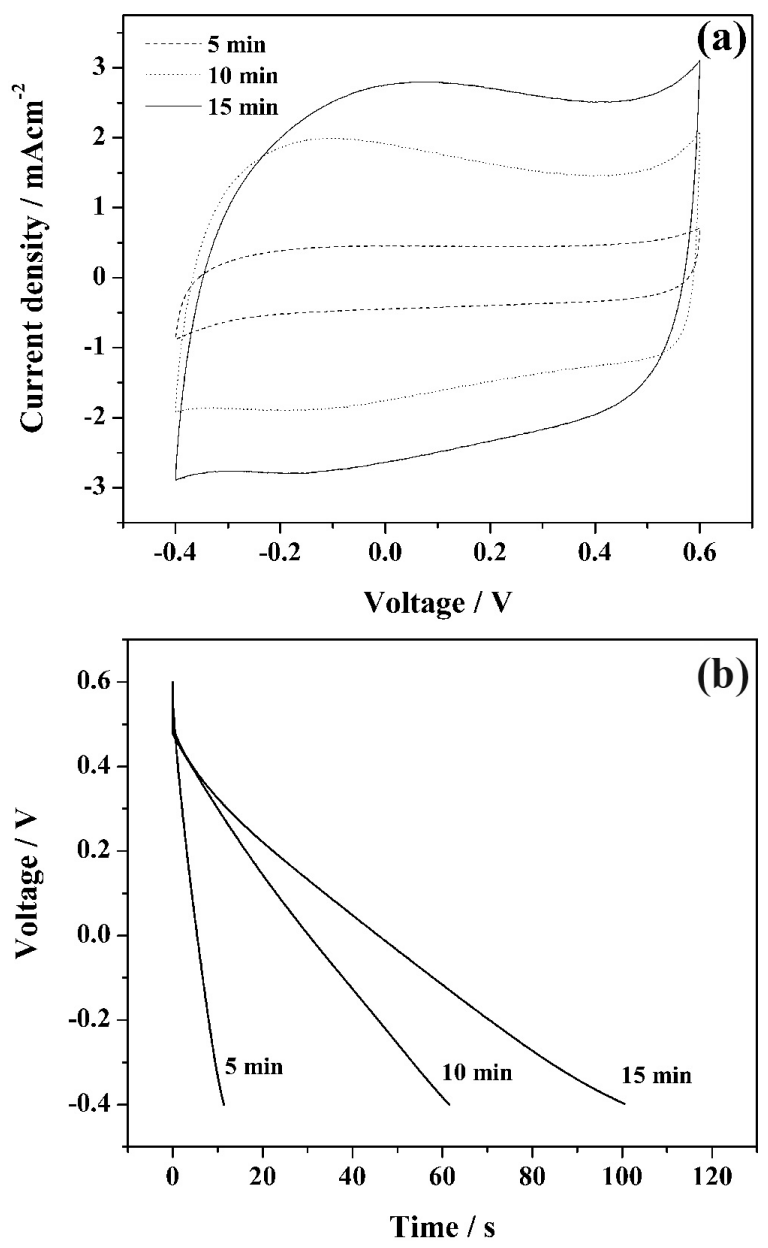

Figure 4.3 (a) CV curves of PPy/C-MEMS single electrodes with different electropolymerization time at $20 \mathrm{mVs}^{-1}$ scan rate. (b) Discharge curves of electrodes at $1 \mathrm{mAcm}^{-2}$ discharge current density. 
PPy can only be p-doped since its n-doping potential is much lower than the reduction potential of molecular solvent-based electrolytes $[15,20]$. The capacitance of PPy films comes from reversible intercalation/deintercalation (doping/dedoping) of electrolyte anions according to the following reactions [15]:

$$
\begin{aligned}
& \mathrm{PPy}+\mathrm{nA}^{-} \rightarrow \mathrm{PPy}^{\mathrm{n}+}\left(\mathrm{A}^{-}\right)_{\mathrm{n}}+\mathrm{ne}^{-} \quad \text { (Charge) } \\
& \mathrm{PPy}^{\mathrm{n}+}\left(\mathrm{A}^{-}\right)_{\mathrm{n}}+\mathrm{ne}^{-} \rightarrow \mathrm{PPy}+\mathrm{nA}^{-} \quad \text { (Discharge) }
\end{aligned}
$$

In these equations, PPy designates a fragment of polypyrrole chain and $\mathrm{A}^{-}$ represents anion. It should be noted that in Eq. (4) and (5), anions ( $\left.\mathrm{A}^{-}\right)$can be primary anions which are integrated into the polymer during electrodeposition $\left(\mathrm{ClO}_{4}{ }^{-}\right)$or secondary anions $\left(\mathrm{Cl}^{-}\right)$that can be involved in electrochemical redox reactions together with or instead of primary anions $[21,22]$. Here, the PPy films deposited on the C-MEMS electrodes are doped with $\mathrm{ClO}_{4}{ }^{-}$ions. During the first discharge half cycle the reaction showed by Eq. (2) occurs and $\mathrm{ClO}_{4}^{-}$leaves the PPy film and is replaced by $\mathrm{Cl}^{-}$in subsequent charge and discharge cycles due to the higher concentration and smaller size of $\mathrm{Cl}^{-}$ions [13]. During charging, $\mathrm{Cl}^{-}$ions diffuse into the PPy film through the path formed by $\mathrm{ClO}_{4}{ }^{-}$undoping. The single electrodes polymerized for 5, 10 and 15 min were also tested with various scan rates from 5 to $100 \mathrm{mVs}^{-1}$. The specific capacitances of electrodes at different CV scan rates are presented in Figure 4.4a.

For all electrodes, specific capacitance decreases with an increase in scan rate. At lower scan rates there is sufficient time for ions to complete the doping/undoping process which results in much higher capacitance compared to the test results at higher scan rates. On the contrary, at high scan rates the change of electric field is faster than charge diffusion, resulting in a relatively lower capacitance. This figure also shows that for all 
scan rates, specific capacitances of the electrode polymerized for $15 \mathrm{~min}$ are higher than electrodes with a lower polymerization time. However, the difference in capacitance is higher in slower scan rates. This is because at the faster scan rates the ions cannot fully diffuse through the thick film of PPy and only a portion of the thickness of the film is accessible to the ions [19].
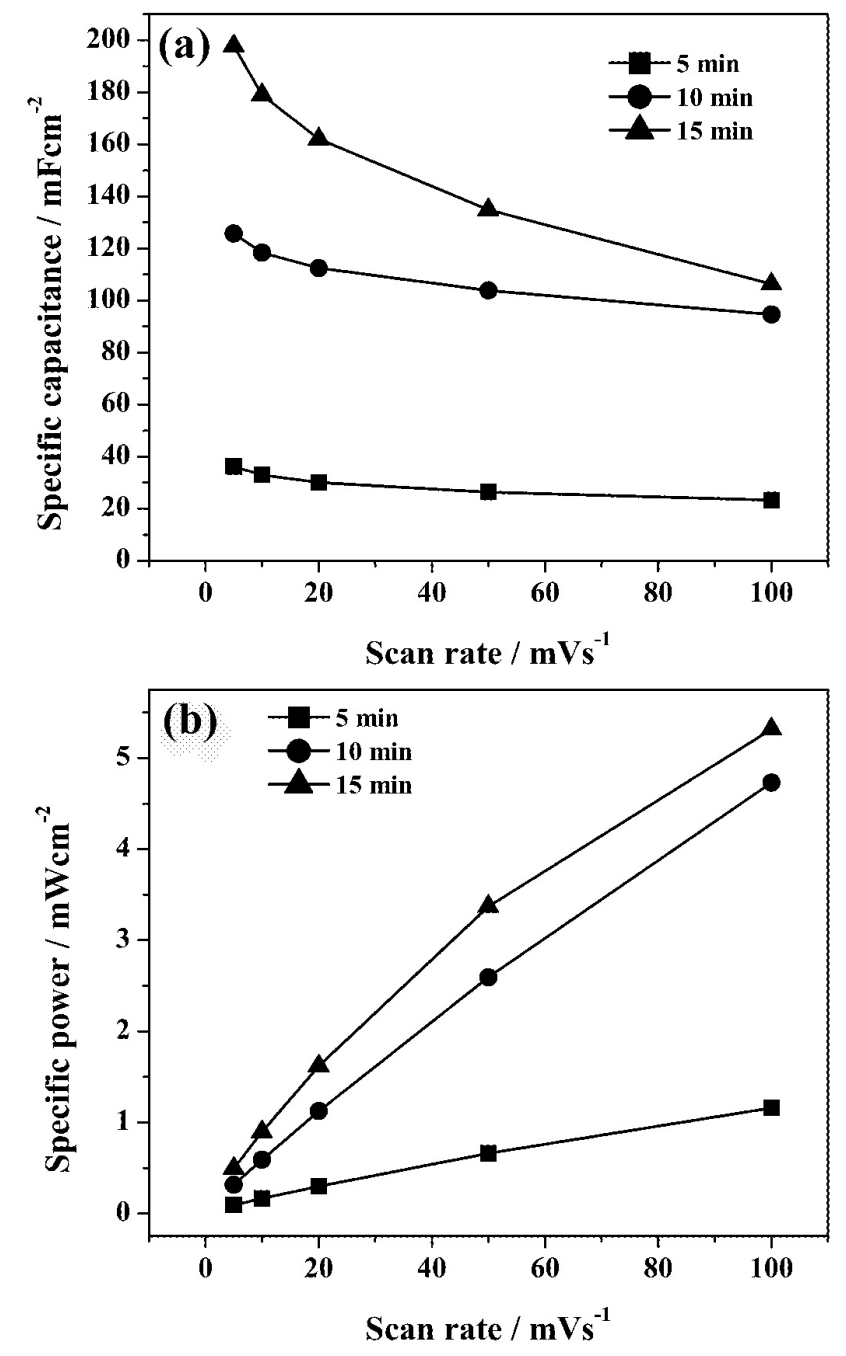

Figure 4.4 (a) Specific capacitance and (b) specific power of three single PPy/C-MEMS electrodes polymerized for 5, 10 and 15 minutes at different scan rates. 
The specific powers of the electrodes at different scan rates are presented in Figure $4.4 \mathrm{~b}$. As can be seen in this figure, specific power increases by increasing both the polymerization time and the scan rates. The electrode polymerized for 15 min has a specific power of $1.62 \pm 0.12 \mathrm{mWcm}^{-2}$ at $20 \mathrm{mVs}^{-1}$ scan rate, higher than the power densities of PPy micro-supercapacitors that have been reported thus far [13]. To show the advantages of the 3D architecture of micro-supercapacitor electrodes, footprint specific capacitance of a 3D PPy/C-MEMS electrode is compared with that of PPy electrodes polymerized on 2D carbon film electrodes in Figure 4.5. For all durations of electropolymerization, the specific capacitances of 3D electrodes are higher than 2D film electrodes. It should be noted that for higher electropolymerization times the difference between capacitance of $3 \mathrm{D}$ and $2 \mathrm{D}$ electrodes are higher. This is due to the better accessibility of electrolyte ions to the thicker PPy film in the 3D structure. The performance of PPy/C-MEMS electrodes were also evaluated in a micro-supercapacitor cell constituting two symmetric electrodes. The CV curves of cells with different PPy electropolymerization times are presented in Figure 4.6. In the symmetric potential window of -0.8 to $0.8 \mathrm{~V}$, and for all polymerization durations, $\mathrm{CV}$ curves present a rectangular shape characteristic of an ideal capacitor. To evaluate the performance of each electrode of each symmetric cell, according to the method described by Sun et al. [13], the CV curve of a symmetric micro-supercapacitor (polymerized for $15 \mathrm{~min}$ ) was divided into four zones by drawing two lines at zero current and zero potential (Figure 4.6). These four zones designate two charging and two discharging parts of the CV curve. The charge or discharge capacitance of each electrode of the symmetric cell can be 
calculated using Eq. (2) for any of the four parts of CV. The calculated charge and discharge capacitances at different scan rates are presented in Table 4.2.

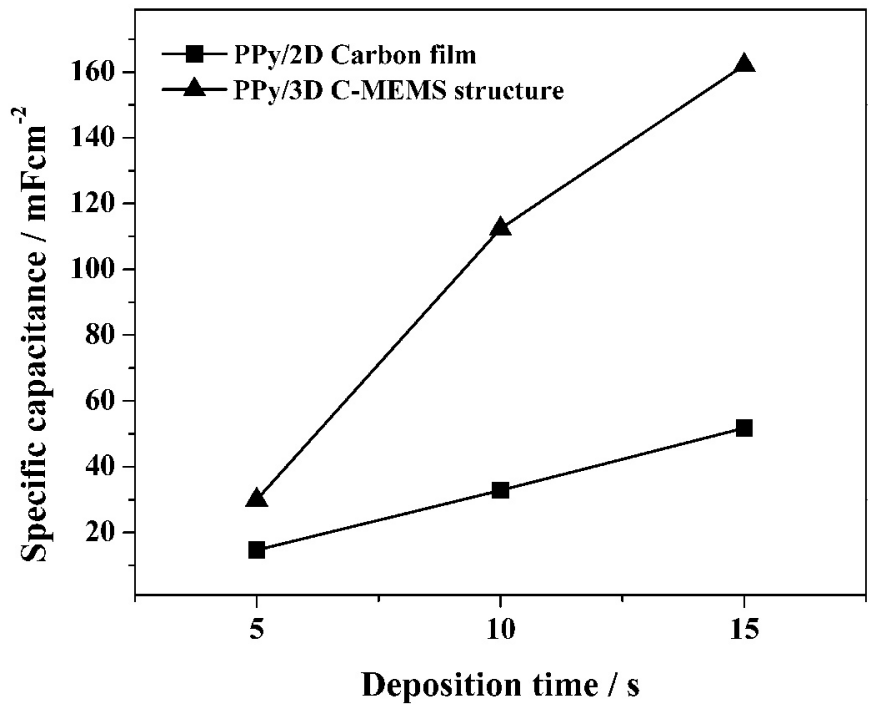

Figure 4.5 Comparison of footprint normalized specific capacitance of PPy/2D carbon film and PPy/3D C-MEMS electrodes.

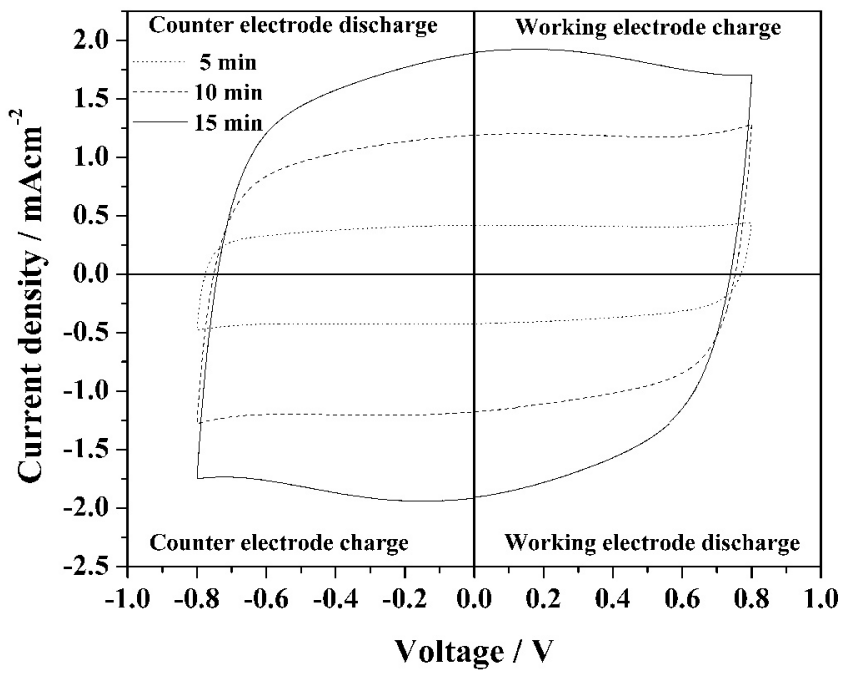

Figure 4.6 CVs of symmetric micro-supercapacitors with different polymerization time. 
Table 4.2 Specific capacitance (capacitance per unit footprint area) of working and counter electrodes of symmetric micro-supercapacitors with different polymerization time at charge and discharge.

\begin{tabular}{ccccc}
\hline & \multicolumn{3}{l}{ Specific capacitance $\left(\mathrm{mFcm}^{-2}\right)$} & \\
\cline { 2 - 5 } & Electrode & & \\
\cline { 2 - 5 } $\begin{array}{c}\text { Polymerization } \\
\text { time (min) }\end{array}$ & $\begin{array}{c}\text { Working electrode } \\
\text { charge }\end{array}$ & $\begin{array}{c}\text { Working electrode } \\
\text { discharge }\end{array}$ & $\begin{array}{c}\text { Counter electrode } \\
\text { charge }\end{array}$ & $\begin{array}{c}\text { Counter electrode } \\
\text { discharge }\end{array}$ \\
\hline 5 & $30.31 \pm 2.58$ & $25.76 \pm 1.53$ & $31.47 \pm 2.43$ & $25.82 \pm 1.55$ \\
10 & $58.32 \pm 3.73$ & $45.81 \pm 2.71$ & $58.33 \pm 3.74$ & $45.80 \pm 2.72$ \\
15 & $89.28 \pm 6.20$ & $67.11 \pm 5.12$ & $89.56 \pm 6.04$ & $68.60 \pm 5.32$ \\
\hline
\end{tabular}

It should be noted in Table 4.2 that for all polymerization times the charging and discharging capacitance of the working and counter electrodes are very similar. This implies that the two electrodes of symmetric C-MEMS/PPy micro-supercapacitors are very similar in geometric surface area. It is also observed in Table 4.2 that for all polymerization times and all electrodes, charge capacitances of electrodes are higher than discharge capacitance and the difference becomes more for thicker PPy films (with higher electropolymerization times). For a symmetric capacitor the total capacitance of the cell can be calculated using Eq. (4.6):

$$
\frac{1}{C}=\frac{1}{C_{1}}+\frac{1}{C_{2}}
$$

If the two electrodes of the supercapacitor have similar capacitance $\left(\mathrm{C}_{1}=\mathrm{C}_{2}\right)$, then we can write $\mathrm{C}_{1}=2 \mathrm{C}$, implying that the capacitance of electrode calculated from $\mathrm{CV}$ with the above mentioned method should be half of the capacitance of single electrodes. Here, the average value of capacitance of the symmetric micro-supercapacitor with 15 min 
polymerization time, i.e. $78.35 \pm 5.67 \mathrm{mFcm}^{-2}$, is slightly lower than half of the capacitance of the PPy/C-MEMS single electrodes calculated from three-electrode tests, i.e., $81.03 \pm 6.2 \mathrm{mFcm}^{-2}$.

Charge-discharge cycles of symmetric micro-supercapacitors are presented in Figure 4.7. The curves show a triangular shape with linear charge and discharge half cycles, typical of supercapacitors. One charge/discharge cycle can also be divided into four parts corresponding to the two electrodes charge and discharge. The specific capacitance calculated from the charge/discharge test of symmetric micro-supercapacitor is $119.05 \pm 6.73 \mathrm{mFcm}^{-2}$ for a device with the PPy deposition time of $15 \mathrm{~min}$.

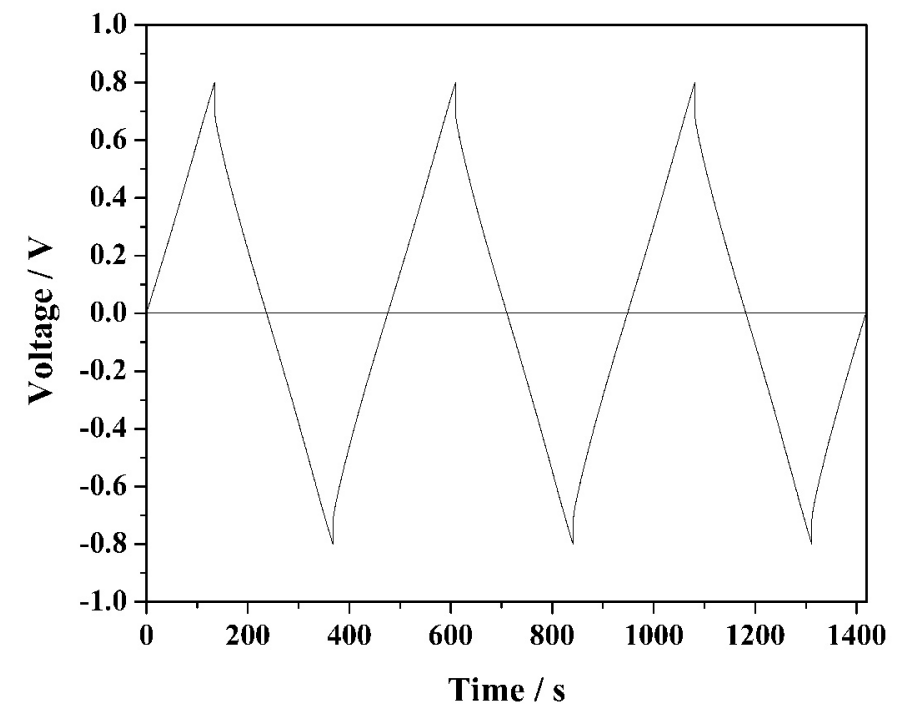

Figure 4.7 Galvonstatic charge/discharge curve of symmetric micro-supercapacitor.

Although testing the device at a symmetric potential window of -0.8 to 0.8 provides a chance to compare the performance of the working and the counter electrodes, the actual operation window of the micro-supercapacitor is 0 to $0.8 \mathrm{~V}$. To investigate the cyclic performance of symmetric PPy/C-MEMS micro-supercapacitors, a microsupercapacitor cell with an electrode polymerization time of 15 min was cycled 1000 
times at a $20 \mathrm{mVs}^{-1} \mathrm{scan}$ rate in a potential window of 0 to $0.8 \mathrm{~V}$. Figure $4.8 \mathrm{a}$ presents the CV curves of the first and 1000th cycle. The specific capacitance of the electrode almost continually fades and after 1000 cycles it reaches almost $56 \%$ of its initial value during the first cycle (Figure 4.8b). Poor cyclic performance is the main drawback of conducting polymer electrodes. Generally, symmetric supercapacitor based on conducting polymer electrodes will have a lower cycle life than those based on carbon. This is due to the difference in the charge storage mechanisms of each. For carbon supercapacitors, charge storage only involves simple ion sorption and desorption. However, in conducting polymer electrodes, doping and undoping of anions or cations will cause a certain amount of volume change of the electrode [15]. This volume change or swelling was reported to cause mechanical failure of the electrode and gradually aggravates its conductive properties $[15,23,24]$. The present study demonstrates that $3 \mathrm{D}$ interdigital C-MEMS electrodes can be utilized as highly effective surface area current collectors for nanostructured PPy films. PPy/C-MEMS electrodes with various electropolymerization times were fabricated and the electrochemical performance of both single electrodes and full cells were tested. At $20 \mathrm{mVS}^{-1}$ scan rate, the maximum capacitance per unit footprint area of $162.07 \pm 12.4 \mathrm{mFcm}^{-2}$ was achieved for the electrodes polymerized for $15 \mathrm{~min}$.

These results show that utilizing $3 \mathrm{D}$ current collectors can increase the specific capacitance of the device in a limited footprint area. The C-MEMS technique provides a more facile rout to fabricated micron sized 3D structures for micro-supercapacitor as compared to other techniques such as micro-molding or DRIE. Moreover, the electrochemical performance of PPy/C-MEMS micro-supercapacitor is improved 
compared to PPy based micro-supercapacitor fabricated through other techniques $[13,25]$. Further improvement of micro-supercapacitor properties is possible by improving cell design to achieve denser arrays of the electrodes with a higher aspect ratio and thus increasing $A_{\text {eff. }}$.
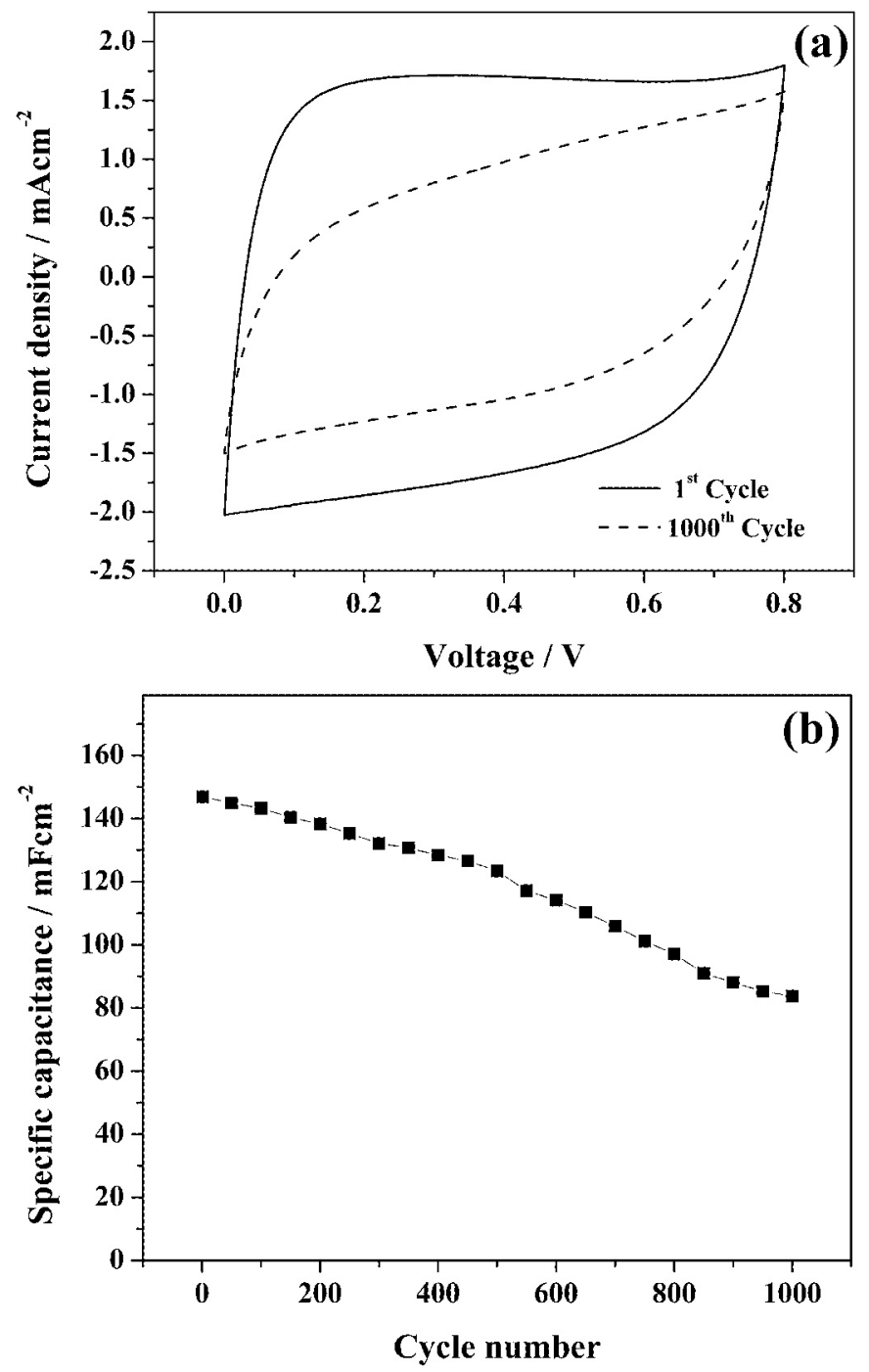

Figure 4.8 (a) $\mathrm{CV}$ curves of a micro-supercapacitor with 15 min electropolymerization time at first and $1000^{\text {th }}$ cycle $\left(20 \mathrm{mVs}^{-1}\right.$ scan rate). (b) Cyclic performance of the device during $1000 \mathrm{CV}$ cycles. 


\subsection{Conclusion}

A symmetric micro-supercapacitor with 3D electrode architecture was designed, fabricated and tested. The 3D carbon structure was fabricated using C-MEMS technique. PPy films were electrochemically deposited on these 3D structures. CV and galvanostatic charge/discharge methods were used to evaluate the performance of single micro electrodes and symmetric micro-supercapacitors. Single electrodes with a PPy polymerization time of 15 min can deliver footprint normalized specific capacitance of $162.07 \pm 12.4 \mathrm{mFcm}^{-2}$ and specific power of $1.62 \pm 0.12 \mathrm{mWcm}^{-2}$ at a $20 \mathrm{mVs}^{-1}$ scan rate. The symmetric micro-supercapacitor delivered specific capacitance of $78.35 \pm 5.67 \mathrm{mFcm}^{-}$

2 at $20 \mathrm{mVs}^{-1}$ scan rate. The increased performance of micro-supercapacitors has enabled them to compete with micro-batteries in a number of applications.

\subsection{References}

[1] B.E. Conway, W.G. Pell, J. Solid State Electrochem. 7 (2003) 637.

[2] P. Simon, Y. Gogotsi, Nat. Mater. 7 (2008) 845.

[3] J. Chmiola, C. Largeot, P.L. Taberna, P. Simon, Y. Gogotsi, Science 328 (2010) 480.

[4] M. Kaempgen, C.K. Chan, J. Ma, Y. Cui, G. Gruner, Nano Lett. 9 (2009) 1872.

[5] D. Pech, M. Brunet, H. Durou, P. Huang, V. Mochalin, Y. Gogotsi, P.-L. Taberna, P. Simon, Nat. Nanotechnol. 5 (2010) 651.

[6] D. Pech, M. Brunet, P.-L. Taberna, P. Simon, N. Fabre, F. Mesnilgrente, V. Conédéra, H. Durou, J. Power Sources 195 (2010) 1266.

[7] M. Beidaghi, W. Chen, C. Wang, J. Power Sources 196 (2011) 2403.

[8] H.R. Byon, S.W. Lee, S. Chen, P.T. Hammond, Y. Shao-Horn, Carbon 49 (2010) 457. 
[9] C.B. Arnold, R.C. Wartena, K.E. Swider-Lyons, A. Pique, J. Electrochem. Soc. 150 (2003) A571.

[10] W. Sun, X. Chen, J. Power Sources 193 (2009) 924.

[11] V. Lee, L. Whittaker, C. Jaye, K.M. Baroudi, D. a Fischer, S. Banerjee, Chem. Mater. 21 (2009) 3905.

[12] F. Chamran, Y. Yeh, H.-S. Min, B. Dunn, C.-J. Kim, J. Microelectromech. Syst. 16 (2007) 844.

[13] W. Sun, R. Zheng, X. Chen, J. Power Sources 195 (2010) 7120.

[14] W. Chen, M. Beidaghi, V. Penmatsa, K. Bechtold, L. Kumari, W.Z. Li, C. Wang, IEEE Trans. Nanotech. 9 (2010) 734.

[15] G. a Snook, P. Kao, A.S. Best, J. Power Sources 196 (2010) 1.

[16] G. Inzelt, V. Kertész, A.-S. Nybäck, J. Solid State Electrochem. 3 (1999) 251.

[17] Y. Liu, Chem. Phys. Lett. 362 (2002) 491.

[18] M.D. Stoller, R.S. Ruoff, Energy Environ. Sci. 3 (2010) 1294.

[19] M. Hughes, G.Z. Chen, M.S.P. Shaffer, D.J. Fray, A.H. Windle, Chem. Mater. 14 (2002) 1610.

[20] K. Naoi, S. Suematsu, A. Manago, J. Electrochem. Soc. 147 (2000) 420.

[21] P.M. Dziewoński, M. Grzeszczuk, J. Phys. Chem. B. 114 (2010) 7158.

[22] A. Kepas, M. Grzeszczuk, Electrochim. Acta 51 (2006) 4167.

[23] E. Frackowiak, V. Khomenko, K. Jurewicz, K. Lota, F. Beguin, J. Power Sources 153 (2006) 413.

[24] V. Khomenko, E. Frackowiak, F. Beguin, Electrochim. Acta 50 (2005) 2499.

[25] J. Sung, J. Power Sources 133 (2004) 312-319. 


\section{ELECTROSTATIC SPRAY DEPOSITION OF GRAPHENE}

NANOPLATELETS FOR HIGH POWER THIN FILM SUPERCAPACITOR

\section{ELECTRODES}

\subsection{Introduction}

Electrochemical capacitors (ECs), also called supercapacitors or ultracapacitors, are energy storage devices with higher energy density than electrostatic or electrolytic capacitors and higher power density than batteries [1]. ECs offer a combination of characteristics such as high power density, long life cycle, wide working temperature range, and safety. These unique properties have made ECs an excellent choice for energy storage in applications such as portable electronics, uninterruptable power supplies, and automotive applications [2]. Based on the charge storage mechanism, ECs are of two types: Electric double layer capacitors (EDLCs) which store charge by adsorption of electrolyte ions on the surface of an electrode with high specific surface area. Different types of high surface area carbon materials are usually used as electrode materials for EDLCs. Pseudo-capacitors store charge by faradic reactions that takes place on the surface or sub-surface of the electrodes. Transition metal oxides and conducting polymers are examples of pseudo-capacitive materials [3]. Among all the desired properties of ECs, high power density, high frequency response and rate capability are very important for their future applications. These properties are particularly significant if the supercapacitors are to be used to provide the peak power in systems with batteries, fuel cells or energy harvesters as the primary energy source. High frequency response is the most important property for supercapacitors if they were to replace electrolytic capacitors in applications such as filtering voltage ripples in line-powered electronics (ac line- 
filtering)[4]. Achieving a high frequency response and rate capability is dependent on the various constituents of a supercapacitor including the electrode materials, electrolyte, and the method of assembly of materials on the current collectors.

Although pseudo-capacitive materials show promising energy density, the slow charge storage mechanism immensely impacts their power density and rate handling capabilities. On the other hand, EDLCs based on carbon materials show high power density and can be charged and charged in very short times $[3,5,6]$. Recently, single layer and multilayer graphene nano sheets have attracted much research interest as electrode materials for supercapacitors. The specific capacitance of graphene nanosheets in the range of 31-264 $\mathrm{Fg}^{-1}$ [7-10] depends on various factors including: preparation method, specific surface area, number of layers in the multilayer graphene, and the electrolyte, among others [7].

In the processes of fabricating supercapacitor electrodes, the method of depositing active materials on current collectors is one of the vital factors that affect the performance of the supercapacitor. Currently two kinds of electrode fabrication methods have been reported to prepare graphene supercapacitor electrodes based on the presence or absence of a polymeric binder to attach the materials to the substrate. The use of polymeric binders has some drawbacks including increased resistivity and addition of dead weight to the electrode [11]. Previous research has demonstrated that binder-free electrodes show considerably improved performance especially with respect to power handling of supercapacitor electrodes [12].

It has been demonstrated that thin film graphene electrodes show exceptional power handling and frequency response [4]. This finding expands the potential 
applications of supercapacitor as they signal the possibility of high power supercapacitors that can even replace electrolytic capacitors in certain applications. Miller et. al [4] reported on vertically oriented graphene grown on nickel foil by means of plasmaenhanced chemical vapour deposition. The supercapacitor showed ac-line filtering capability at $120 \mathrm{~Hz}$ and a resistance capacitance (RC) time constant of $200 \mu \mathrm{s}$. However, the slow graphene growth rate and the use vacuum process equipment make this process very costly.

Several methods have been reported to fabricate the binder-free electrodes of graphene nanosheets including the vacuum filtration method [13], chemical vapor deposition (CVD) [4], electrophoretic deposition (EPD)[14], and layer-by-layer (LBL) assembly[15]. Among these techniques CVD, LBL and EPD can be easily controlled to deposit a thin film of graphene. However, these methods are time consuming and costly for industrial production of electrodes. The future application of graphene as supercapacitor material depends on development of versatile and cost effective electrode fabrication methods.

In this paper, a versatile and cost effective technique, electrostatic spray deposition (ESD), has been used to fabricate thin films of graphene. In this method, a liquid precursor solution feeding through a nozzle is atomized at the tip of the nozzle by applying high electric potential between the nozzle and a heated substrate [16-18]. The atomized solution lands on the substrate and upon evaporation of the solvent, a solid or porous film can be formed. The morphology and thickness of the deposited film can be controlled by adjusting the deposition parameters such as the flow rate, applied potential, substrate temperature, and the composition of the precursor solution [18]. Usually, the 
precursor is a compound which can be decomposed to the desired material at a high temperature during deposition or calcination after the deposition. For instance ESD has been used to deposit thin films of metal oxides from metal salt solutions [16,17]. The material to be deposited such as CNT [19] can also be sprayed directly onto the substrate from a suspension of the material in an appropriate solvent. There are few reports in the literature on ESD deposition of graphene and graphene oxide [20,21]. It was shown that the combination of the small droplet size and rapid evaporation of the solvent upon reaching the preheated substrate avoids the concentration process that takes place upon slow solvent evaporation [21]. This results in reduced restacking graphene sheets during the position and thus provides higher accessibility of the electrodes.

In this study, for the first time, we evaluate the performance of ESD deposited graphene film for supercapacitor application. A commercially available graphene nanoplatelet (GNP) with medium specific surface area was used as the electrode material. The ESD deposition results in uniform deposited film with an open pore structure. The electrochemical properties of the electrodes were evaluated by cyclic voltammetry (CV), galvanostatic charge-discharge (CD) and electrochemical impedance spectroscopy (EIS). The results show that while the electrodes have an acceptable specific capacitance relative to the medium specific surface area of the starting materials, they show excellent power handling ability and frequency response which is the result of the thin film and open pore structure of the electrodes.

\subsection{Experimental}

GNPs were commercially obtained from Cheaptubes Inc (Brattleboro, VT, USA). All other chemicals were purchased from Sigma-Aldrich (St Louis, MO, USA). GNPs 
have specific surface area of $600-750 \mathrm{~m}^{2} \mathrm{~g}^{-1}$ and average platelet diameter of less than 2 $\mu \mathrm{m}$ and were used without any purification. The experimental setup and details of the ESD process used in this work has been reported previously [22]. Figure 5.1a schematically shows the ESD set up. In a typical sample fabrication processes, $6 \mathrm{mg}$ of GNP was added to $20 \mathrm{ml}$ of 1,2-propanediol and sonicated for $30 \mathrm{~min}$ with an ultrasonic probe in an ice bath for $30 \mathrm{~min}$ to form a $0.3 \mathrm{mg} / \mathrm{ml} \mathrm{GNP}$ solution. In the next step, the solution was fed to a stainless needle through a syringe pump at feeding rate of 0.5 or 3 $\mathrm{ml} / \mathrm{h}$ and a voltage of $4-5 \mathrm{kV}$ was applied between the needle and stainless steel substrate which was placed on a hotplate and heated at $250^{\circ} \mathrm{C}$. After the deposition, the thicknesses of the thin films were measured by using a profilometer. The deposition times were adjusted to reach film thicknesses of 1 and $6 \mu \mathrm{m}$. Figure $5.1 \mathrm{~b}$ schematically shows the structure of deposited GNP films. Electron microscopy was carried out on a JEOL 7000 field-emission scanning electron microscope (FE-SEM) and a TECNAI-F20 FEG transmission electron microscope (TEM). The electrochemical tests were carried out on a VMP3 multichannel potentiostatic (VMP3, Bio-Logic, USA). The electrochemical performance of the electrodes was studied in a three electrode cell using a Pt wire and $\mathrm{Ag} / \mathrm{AgCl}$ electrode as counter and reference electrodes, respectively. A $1 \mathrm{M} \mathrm{Na}_{2} \mathrm{SO}_{4}$ solution was used as the electrolyte. The electrochemical impedance spectroscopy (EIS) measurements were conducted by applying an alternating voltage of $10 \mathrm{mV}$ to electrode at its open circuit voltage from $100 \mathrm{kHz}$ to $50 \mathrm{mHz}$.

\subsection{Results and Discussion}

Figures $5.2 \mathrm{a}$ and $\mathrm{b}$ present cross-section and top-view FE-SEM images of deposited GNP films. As shown in Figure 5.2b, GNPs interacted with each other to form 
an open pore structure, through which electrolyte ions can diffuse and reach the surface of underlying GNPs [23]. Figure 5.2c shows a TEM image of as-deposited GNPs. It is evident that GNPs are corrugated and scrolled. This is a part of the intrinsic nature of graphene nanosheets, which results from the fact that the 2D membrane structure becomes thermodynamically stable via bending [24].

(a)

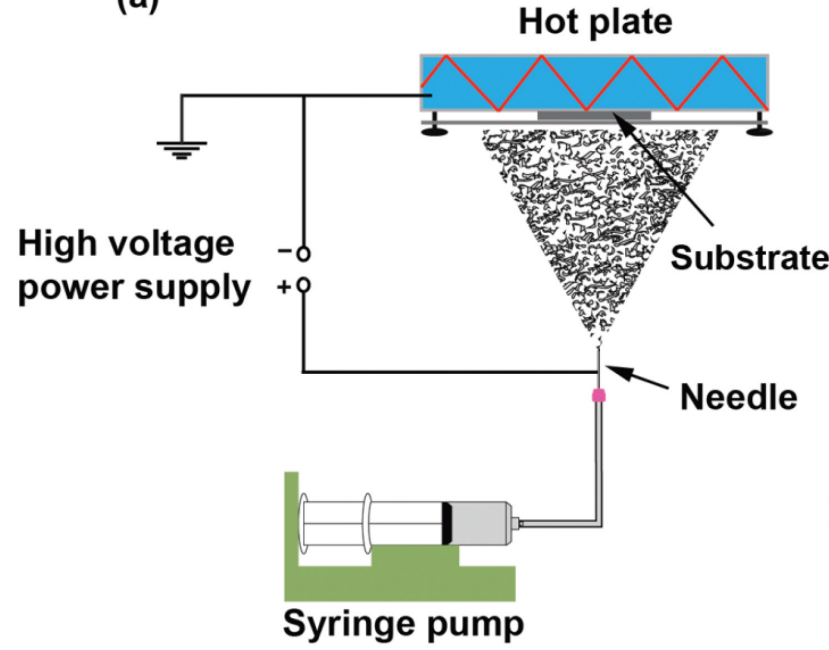

(b)

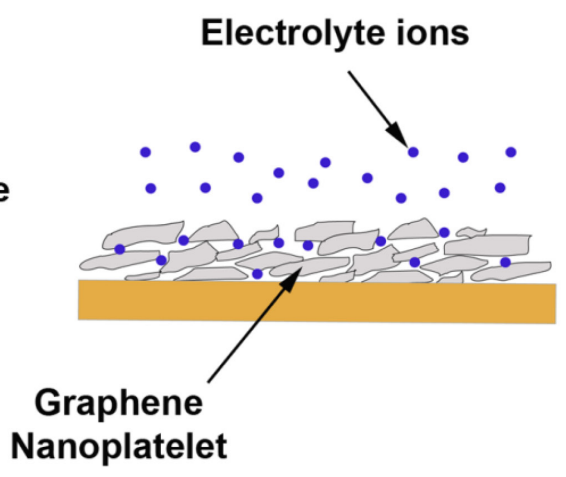

Figure 5.1 (a) Schematic drawing of ESD set-up that was used in this study. (b) Schematic drawing showing the deposited thin GNP film with open pore structure with easy accessibility of electrolyte ions.

The as-prepared thin film GNP electrodes show a moderate specific capacitance with excellent high power handling. Figure 5.3a-d show the CV curves of 1 and $6 \mu \mathrm{m}$ thick electrodes at different scan rates ranging from 0.1 to $20 \mathrm{Vs}^{-1}$. Both electrodes show capacitive properties at scan rates as high as $20 \mathrm{Vs}^{-1}$. For the $1 \mu \mathrm{m}$ thick electrode, the $\mathrm{CV}$ curves exhibit rectangular shapes for scan rates as high as $10 \mathrm{Vs}^{-1}$ which shows excellent capacitive properties. At the very high scan rate of $20 \mathrm{Vs}^{-1}$ the shape of $\mathrm{CV}$ deviates from the rectangular shape which indicated a more resistive behavior at this scan 
rate. For the electrode with $6 \mu \mathrm{m}$ thickness, the $\mathrm{CV}$ curves have an ideal rectangular shape at 0.1 and $1 \mathrm{Vs}^{-1}$. However, at scan rates higher than $5 \mathrm{Vs}^{-1}$ the shape of CVs indicate more resistive contribution of electrodes. At high scan rate of $20 \mathrm{Vs}^{-1}$ the resistive behavior of the electrodes is dominant and electrodes show small specific capacitance.
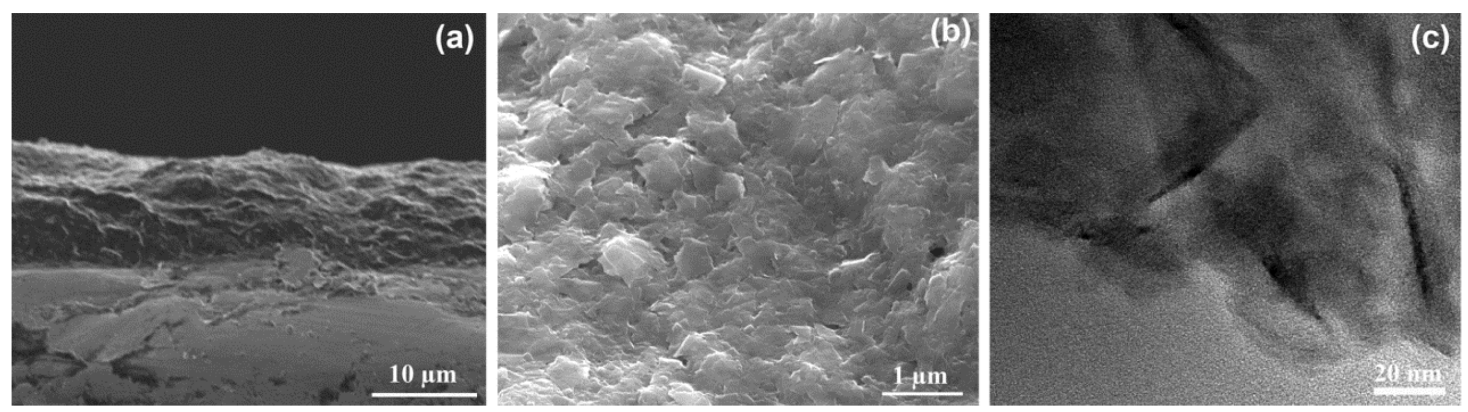

Figure 5.2 SEM image of the deposited GNP (6 $\mu \mathrm{m}$ thick) film (a) cross section view (b) top view. (c) TEM image of as received GNP.

At a scan rate of $0.1 \mathrm{Vs}^{-1}$ the specific capacitance of $1 \mu \mathrm{m}$ thick electrode is about $52 \mathrm{Fg}^{-1}$. At this scan rate the specific capacitance of $6 \mu \mathrm{m}$ thick electrode is about $49 \mathrm{Fg}^{-1}$. This shows a medium specific capacitance compared to reported data for specific capacitance of graphene $\left(36-264 \mathrm{Fg}^{-1}\right)$. However, it should be noted that the GNP that was used in this study has a medium surface area of $600-750 \mathrm{~m}^{2} \mathrm{~g}^{-1}$ and consists of multilayers and its specific capacitance is reasonable when compared to the reported capacitance of multilayer graphene. It is also worth noting that in the process of making the graphene film electrodes no surfactant materials were used to disperse the graphene in the deposition solution. Recently, Brownson et al.[25], showed that the presence of surfactant could affect the specific capacitance of graphene electrodes. For instance, the 
specific capacitance of about $148 \mathrm{Fg}^{-1}$ for graphene dispersed in the processing solution with the help of surfactant, dropped to about $63 \mathrm{Fg}^{-1}$ without using the surfactant[25].
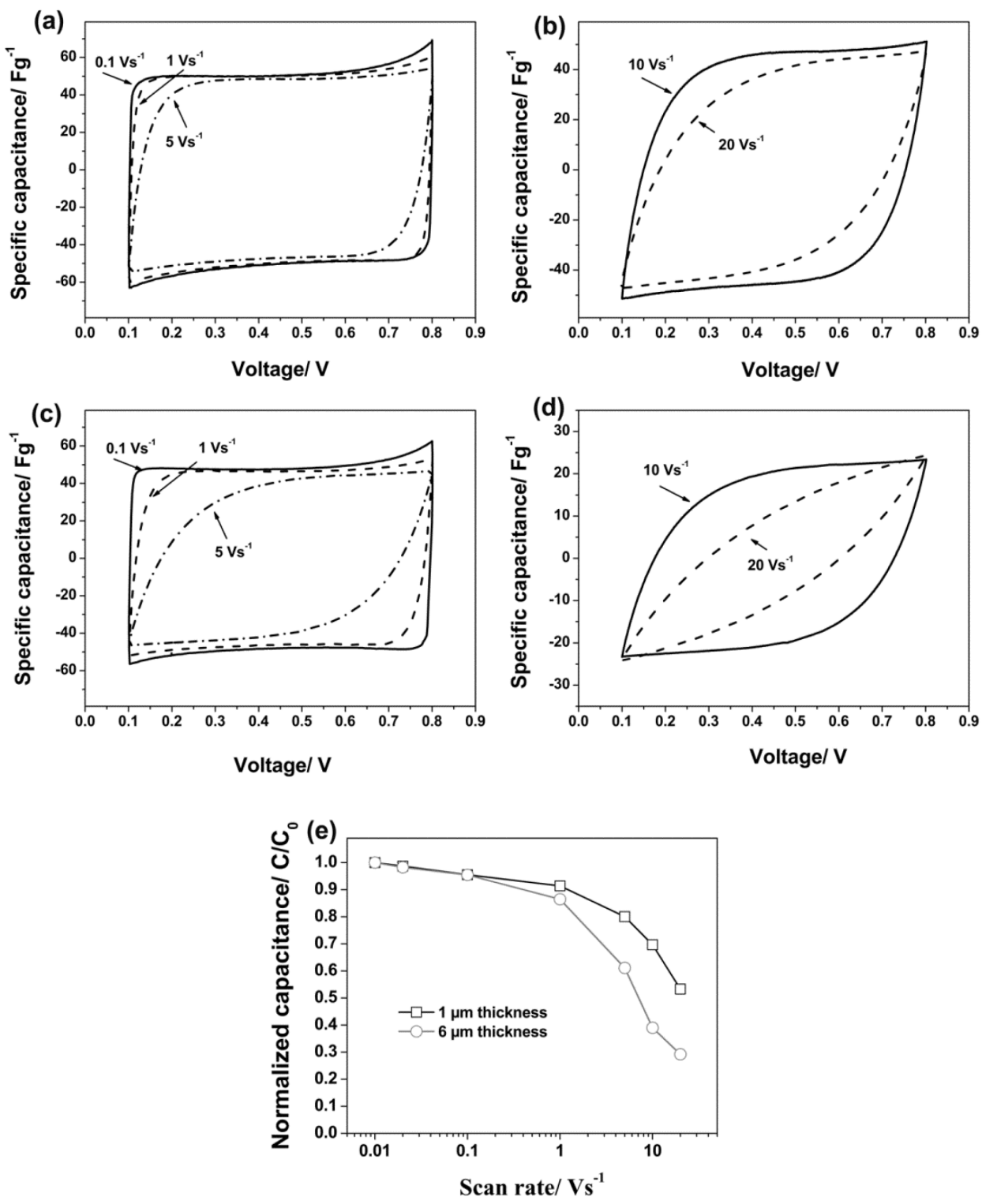

Figure 5.3 Cyclic voltammograms recorded in $1 \mathrm{M} \mathrm{Na}_{2} \mathrm{SO}_{4}$ (vs. $\mathrm{Ag} / \mathrm{AgCl}$ ) of GNP electrodes at various scan rates (a, b) $1 \mu \mathrm{m}$ thick electrode (c, d) $6 \mu \mathrm{m}$ thick electrode. (e) Plot of normalized capacitance vs. scan rate for electrode with 1 and $6 \mu \mathrm{m}$ thickness. 
To investigate the rate capability of graphene film electrodes, the normalized specific capacitance of the films at scan rates between 0.01 to $20 \mathrm{Vs}^{-1}$ were calculated and shown in Figure 5.3e. The drop in capacitance of the thinner electrode with respect to specific capacitance at $0.1 \mathrm{Vs}^{-1}$ is only $20 \%$ at a high scan rate of $5 \mathrm{Vs}^{-1}$. The drop in capacitance at a $5 \mathrm{Vs}^{-1}$ scan rate is higher for the thicker electrode (38\%) but is still lower than most of the reported thin film electrodes. For both electrodes the capacitance drops slowly with further increase in the scan rate, and at a very high scan rate of $20 \mathrm{Vs}^{-1}$, around $53 \%$ of specific capacitance of electrodes with $1 \mu \mathrm{m}$ thickness and $30 \%$ of specific capacitance of the electrodes with $6 \mu \mathrm{m}$ thickness is retained. These results show the excellent rate capability and power handling of graphene thin film electrodes. Moreover, it can be concluded that the thickness of the electrode has strong influence on the power handling ability and highlights the importance of thin film electrodes for applications that require high rate capability.

The properties of the thin film electrodes were also studied by galvanostatic charge/discharge tests. Figure 5.4a-d show charge/discharge curves of the electrodes at various current densities. At all current density the curves show triangle shapes indicating ideal capacitive behavior. The $i R$ drop, the sudden voltage drop at the beginning of discharge curves, is very small for both types of electrodes at low discharge current densities of 1-10 $\mathrm{Ag}^{-1}$. At higher discharge current densities the $i R$ drop increases, but is still relatively small. At discharge current density of $50 \mathrm{Ag}^{-1}$ the $i R$ drop for the electrode with $1 \mu \mathrm{m}$ thickness is about $0.03 \mathrm{~V}$ and it increases to $0.09 \mathrm{~V}$ at higher discharge current density of $200 \mathrm{Ag}^{-1}$. It should be noted that discharge current density of $200 \mathrm{Ag}^{-1}$ is much higher than discharge rates that are usually used to test the supercapacitor electrodes 
(between 0.1 to $10 \mathrm{Ag}^{-1}$ ) [26]. Figure $5.4 \mathrm{~d}$ presents the specific capacitance of the electrodes at various discharge current densities.
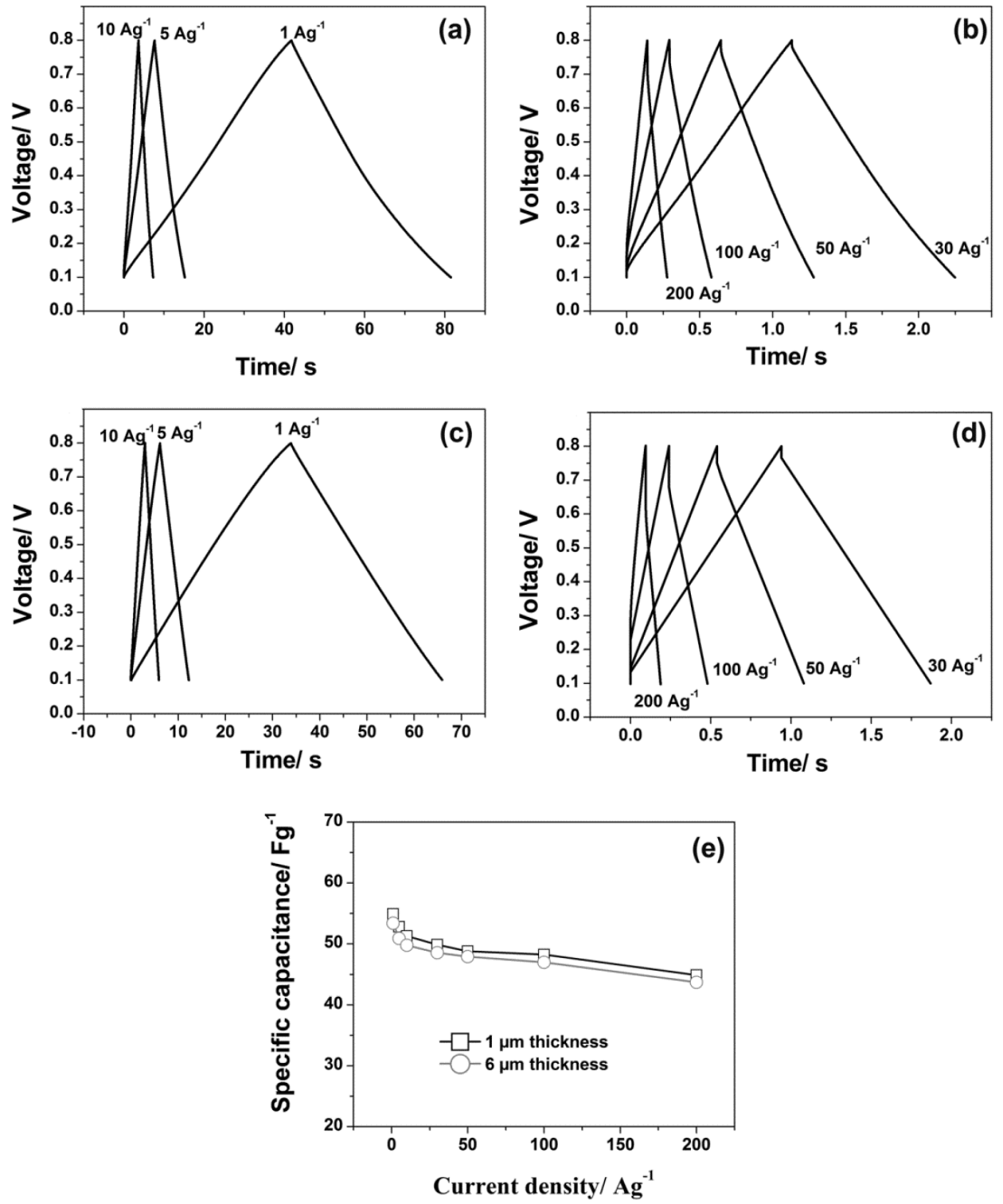

Figure 5.4 Galvonstatic charge/discharge curves of GNP thin film electrode at various current densities (a, b) $1 \mu \mathrm{m}$ thick electrode (c, d) $6 \mu \mathrm{m}$ thick electrode. (e) Specific capacitances obtained from galvanostatic charge/discharge measurements at various current densities.

At the low discharge rate of $1 \mathrm{Ag}^{-1}$ the specific capacitance of the GNP films with 1 and $6 \mu \mathrm{m}$ thicknesses are about 55 and $53 \mathrm{Fg}^{-1}$, respectively. For both electrode 
thicknesses approximately $82 \%$ of the specific capacitance at the $1 \mathrm{Ag}^{-1}$ discharge rate was achieved at very high discharge rate of $200 \mathrm{Ag}^{-1}$. These results, in agreement with results obtained from cyclic voltammetry, confirm exceptional high rate handling ability of the GNP films fabricated through the ESD technique. The rate capability of GNP film electrodes is very similar to that of onion like carbon (OLC) which has lower specific capacitance compared to $\mathrm{GNP}\left(\leq 40 \mathrm{Fg}^{-1}\right)[11,12,27]$.

Further investigation of electrodes was performed using electrochemical impedance spectroscopy (EIS). For a more informative analysis of EIS results, we transformed the impedance data to the complex capacitance following the approach that is described by Taberna et al.[28]. In this approach, the real part $\left(C^{\prime}\right)$ and the imaginary part $\left(C^{\prime \prime}\right)$ of the capacitance are both function of the frequency and can be extracted from impedance data according to the equations (5.1) and (5.2)[28,29].

$$
\begin{aligned}
& C^{\prime \prime}=\frac{Z^{\prime}(f)}{2 \pi f|Z(f)|^{2}} \\
& C^{\prime}=\frac{Z^{\prime \prime}(f)}{2 \pi f|Z(f)|^{2}}
\end{aligned}
$$

where $Z(f)$ is the complex impedance at frequency $f$, and $Z^{\prime}(f)$ and $Z^{\prime \prime}(f)$ are real and imaginary part of the complex impedance, respectively.

Figures $5.5 \mathrm{a}$ and $\mathrm{b}$ show the resulting graphs for the electrodes with 1 and $6 \mu \mathrm{m}$ thickness, respectively. For the real part of the capacitance, an increase in capacitance is observed with a decrease in ac frequency and the capacitance value approaches to the value obtained from $\mathrm{CV}$ tests. 

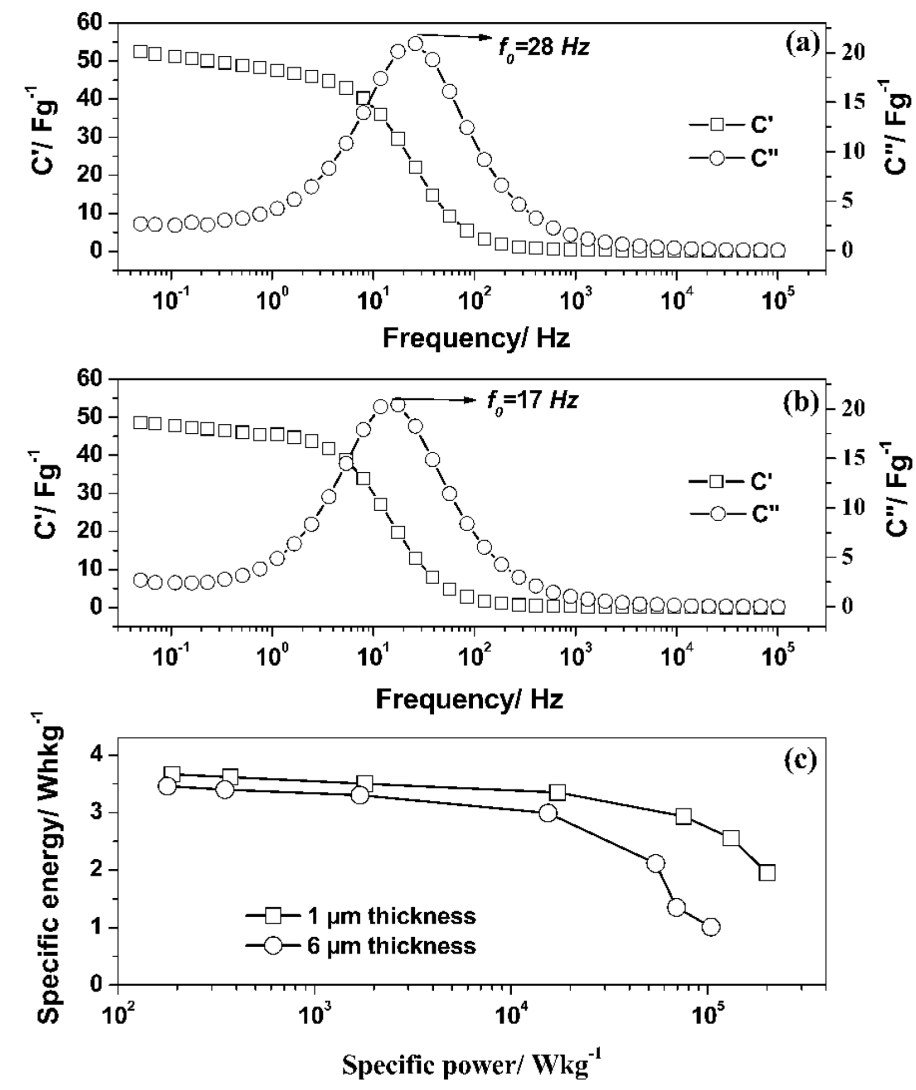

Figure 5.5 Plots of complex real (C') and imaginary ( $\left.\mathrm{C}^{\prime \prime}\right)$ capacitance as a function of frequency for (a) electrode with $1 \mu \mathrm{m}$ thickness (b) electrode with $6 \mu \mathrm{m}$ thickness. (c) Plots of utilizable specific energy Vs. power density (Ragone plot) of the thin film GNP electrodes.

For imaginary capacitance plots, a peak shape curve is obtained. The rate capability of the electrode can be characterized in the real capacitance plots by observing the frequency range where the capacitance increases. The rate capability can also be quantitatively evaluated from the frequency of capacitive peak in imaginary capacitance plots [30]. This characteristic frequency $\left(\mathrm{f}_{0}\right)$ marks the point at which the phase angle of the impedance is $-45^{\circ}$ (resistive and capacitive impedance are equal) and the electrode is discharged at $50 \%$ efficiency [27,28]. The characteristic frequency was estimated to be around 28 and $17 \mathrm{~Hz}$ for the electrodes with 1 and $6 \mu \mathrm{m}$ thicknesses, respectively. The 
corresponding time constants $\mathrm{T}_{0}\left(\mathrm{~T}_{0}=1 / \mathrm{f}_{0}\right)$ were about 36 and $59 \mathrm{~ms}$, respectively. For comparison, it is worth noting that many of reported supercapacitor electrodes with high rate capability have much higher time constants. For instance, the calculated time constant in aqueous electrolyte is $700 \mathrm{~ms}$ for MCNT [27], $1.1 \mathrm{~s}$ for OLC [27], and $379 \mathrm{~ms}$ for carbide derived carbon (CDC) nano-felts [11]. There are some reports in the literature about supercapacitor devices with lower time constants. A $7 \mu \mathrm{m}$ thick film of OLC arranged in interdigital micro-supercapacitor device showed a $26 \mathrm{~ms}$ time constant [12]. However, OLCs have lower specific capacitance compared to GNPs that are used here $\left(<40 \mathrm{Fg}^{-1}\right)$. To the best of our knowledge the highest rate capability that has been reported so far is for that of supercapacitor with $0.6 \mu \mathrm{m}$ thick vertically oriented graphene sheets that could achieve a time constant of $200 \mu$ s at a high frequency of $120 \mathrm{~Hz}$. At this frequency the capacitance of a device with $2 \mathrm{~cm}^{2}$ surface area was $175 \mu \mathrm{F}$, making the capacitance of a single electrode about $359 \mu \mathrm{F}$ (normalized capacitance of $175 \mu \mathrm{Fcm}^{-2}$ ). It is worth noting that at higher frequencies of $125 \mathrm{~Hz}$ the supercapacitor electrodes reported in this study show a normalized capacitance of 850 and $2000 \mu \mathrm{Fcm}^{-2}$ for the film thickness of 1 and $6 \mu \mathrm{m}$, respectively. A combination of binder-free deposition technique, thin film configurations of GNPs with an open pore structure, and high conductivity of GNPs are responsible for the observed high power handling ability of the electrodes. A Ragone plot calculated from CV results (Fig. 5c) reveals that the $1 \mu \mathrm{m}$ thick electrode shows a specific energy of $3.66 \mathrm{Whkg}^{-1}$ at $188.50 \mathrm{Wkg}^{-1}$ specific power. The specific energy slowly decreases as specific power increases and at very high specific power of $200.90 \mathrm{kWkg}^{-1}$, the specific energy of $1.95 \mathrm{Whkg}^{-1}$ was achieved. 


\subsection{Conclusions}

In summary, for the first time we have utilized the ESD technique to fabricate binderfree thin films of GNP for supercapacitor application. A commercially available multilayer GNP was selected to demonstrate the fabrication technique. The electrodes show excellent high rate handling ability as it was shown by various electrochemical testing techniques. Electrodes with $1 \mu \mathrm{m}$ thickness show a much better rate handling ability compared to electrodes with $6 \mu \mathrm{m}$, highlighting the effect of electrode thickness on the power performance of supercapacitors. At the low discharge rate of $1 \mathrm{Ag}^{-1}$ the specific capacitance of the GNP films with 1 and $6 \mu \mathrm{m}$ thicknesses are about 55 and 53 $\mathrm{Fg}^{-1}$, respectively. The specific capacitance of the electrodes is lower than that of the high quality lab produced single layer graphene. We anticipate that by using graphene materials with higher surface area and optimizing the depositions conditions higher specific capacitance is feasible. Nonetheless, the GNP electrodes studied here are an excellent candidate for the applications that require high power and moderate energy density. EIS experiments indicate excellent frequency response of the electrodes and the low RC time constants of 36 and $59 \mathrm{~ms}$ was recorded for electrodes with 1 and $6 \mu \mathrm{m}$

thickness, respectively. Additionally, the demonstrated fabrication process (ESD) is a low-cost technique that could potentially be employed to fabricate thin film electrodes of various kinds of graphene and its composites.

\subsection{References}

[1] B.E. Conway, Electrochemical Supercapacitors: Scientific Fundamentals and Technological Applications, Kluwer Academic/Plenum Publishers, New York, 1997.

[2] J.R. Miller, A.F. Burke, Electrochem. Soc. Interf. 17, 53-57 (2008). 
[3] P. Simon, Y. Gogotsi, Nat. Mater. 7 (2008) 845-54.

[4] J.R. Miller, R. a Outlaw, B.C. Holloway, Science 329 (2010) 1637-9.

[5] A. Pandolfo, A. Hollenkamp, J. Power Sources 157 (2006) 11-27.

[6] V. Obreja, Physica E 40 (2008) 2596-2605.

[7] D. a. C. Brownson, D.K. Kampouris, C.E. Banks, J. Power Sources 196 (2011) 4873-4885.

[8] H.-W. Wang, Z.-A. Hu, Y.-Q. Chang, Y.-L. Chen, Z.-Q. Lei, Z.-Y. Zhang, Y.-Y. Yang, Electrochim. Acta 55 (2010) 8974-8980.

[9] T. Lu, Y. Zhang, H. Li, L. Pan, Y. Li, Z. Sun, Electrochim. Acta 55 (2010) 41704173.

[10] Z.-S. Wu, W. Ren, D.-W. Wang, F. Li, B. Liu, H.-M. Cheng, ACS Nano 4 (2010) $5835-42$

[11] V. Presser, L. Zhang, J.J. Niu, J. McDonough, C. Perez, H. Fong, Y. Gogotsi, Adv. Energy Mater. 1 (2011) 423-430.

[12] D. Pech, M. Brunet, H. Durou, P. Huang, V. Mochalin, Y. Gogotsi, P.-L. Taberna, P. Simon, Nat. Nanotechnol. 5 (2010) 651-654.

[13] A. Yu, I. Roes, A. Davies, Z. Chen, Appl. Phys. Lett. 96 (2010) 253105.

[14] Y. Chen, X. Zhang, P. Yu, Y. Ma, J. Power Sources 195 (2010) 3031-3035.

[15] H.R. Byon, S.W. Lee, S. Chen, P.T. Hammond, Y. Shao-Horn, Carbon 49 (2010) 457-467.

[16] S. Sokolov, B. Paul, E. Ortel, A. Fischer, R. Kraehnert, Langmuir 27 (2011) 19727.

[17] Y. Yu, L. Gu, A. Dhanabalan, C.-H. Chen, C. Wang, Electrochimica Acta 54 (2009) 7227-7230.

[18] a. Jaworek, A.T. Sobczyk, J. Electrost. 66 (2008) 197-219.

[19] J. Kim, K. Nam, S. Ma, K. Kim, Carbon 44 (2006) 1963-1968.

[20] P. Han, Y. Yue, Z. Liu, W. Xu, L. Zhang, H. Xu, S. Dong, G. Cui, Energy \& Environmental Science (2011). 
[21] S. Gilje, S. Han, M. Wang, K.L. Wang, R.B. Kaner, Nano Letters 7 (2007) 3394-8.

[22] W. Chen, M. Beidaghi, V. Penmatsa, K. Bechtold, L. Kumari, W.Z. Li, C. (Peggy) Wang, IEEE Transactions on Nanotechnology 9 (2010) 734-740.

[23] W. Lv, D.-M. Tang, Y.-B. He, C.-H. You, Z.-Q. Shi, X.-C. Chen, C.-M. Chen, P.X. Hou, C. Liu, Q.-H. Yang, ACS Nano 3 (2009) 3730-6.

[24] G. Wang, X. Shen, J. Yao, J. Park, Carbon 47 (2009) 2049-2053.

[25] D. a C. Brownson, C.E. Banks, Chem. Commun. (Cambridge, England) (2011) 35 .

[26] G. Wang, L. Zhang, J. Zhang, Chemical Society Reviews 41 (2011) 797-828.

[27] C. Portet, G. Yushin, Y. Gogotsi, Carbon 45 (2007) 2511-2518.

[28] P.L. Taberna, P. Simon, J.F. Fauvarque, J. Electrochem. Soc. 150 (2003) A292.

[29] J.H. Jang, S. Yoon, B.H. Ka, Y.-H. Jung, S.M. Oh, J. Electrochem. Soc. 152 (2005) A1418.

[30] J.H. Jang, A. Kato, K. Machida, K. Naoi, J. Electrochem. Soc. 153 (2006) A321. 


\section{MICRO-SUPERCAPACITORS BASED ON INTERDIGITAL ELECTRODES OF REDUCED GRAPHENE OXIDE AND CNT COMPOSITES}

\subsection{Introduction}

Recent development in miniaturized electronic devices has increased the demand for power sources that are sufficiently compact and can potentially be integrated on a chip with other electronic components. Miniaturized electrochemical capacitors (EC) or micro-supercapacitors have great potential to complement or replace batteries and electrolytic capacitors in a variety of applications [1-10]. Among all the desired properties of a micro-supercapacitor device, high power density and more importantly high frequency response and rate capability are crucial for their future applications. These properties are particularly important if the micro-supercapacitors were to be coupled with micro-batteries, micro-fuel cells, and energy harvesters to provide peak power; or if they were to replace electrolytic capacitors in applications such as filtering voltage ripples in line-powered electronics (ac line-filtering) [11]. Achieving a high frequency response and rate capability is dependent on the various constituents of a supercapacitor including the electrode materials, electrolyte, the method of assembly of materials on the current collectors, and the architecture of the device.

ECs are categorized into two types based on their energy storage mechanism, electrical double-layer capacitors (EDLCs) and pseudo-capacitors. EDLCs store charge by adsorption of electrolyte ions on the surface of an electrode with high specific surface area. Different types of high surface area carbon materials are usually used as electrode materials for EDLCs. Pseudo-capacitors store charge by faradic reactions that takes place on the surface or sub-surface of the electrodes. Metal oxides such as Manganese oxide [2] 
and conducting polymers such as Polypyrrole (PPy)[3] and Polyaniline (PANI) [4] are widely reported as pseudo-capacitive materials. Although pseudo-capacitive materials show promising volumetric capacitance, the slow charge storage mechanism immensely impacts their frequency response and rate handling capabilities. Carbon nanomaterial such as, activated carbon (AC) [5] carbide derived carbon (CDC) [6,7], onion-like carbon (OLC) [8], carbon nanotube (CNT) [9], and graphene [10] have been used to fabricate EDLC micro-supercapacitors. Micro-supercapacitors based on AC show medium stack capacitance, however due to the use of polymeric binders and limited ion transfer in the porous network of the electrode materials, AC micro-supercapacitors show relatively poor frequency response [5]. The CDC based micro-supercapacitors show high volumetric capacitance at low scan rates (about $180 \mathrm{Fcm}^{-3}$ volumetric capacitance of one electrode at $20 \mathrm{mVs}^{-1}$ ), however the capacitance drops to almost half of its initial value by increasing the scan rate to $500 \mathrm{mVs}^{-1}$, suggesting the poor rate handling capability of these micro-supercapacitors [7]. Among all the reported EDLC micro-supercapacitors, those based on OLCs are particularly notable as they offer ultra-high power handling capability with a resistance capacitance (RC) time constant of only $26 \mathrm{~ms}$ [8]. The combination of micrometer-sized interdigital electrode design with a binder free deposition technique and the non-porous morphology of OLC materials was responsible for the excellent frequency response of OLC based micro-supercapacitors. The drawback of OLC based micro-supercapacitors is their modest specific capacitance $\left(1.7 \mathrm{mFcm}^{-2}\right)$ and their high temperature processing requirements $\left(\sim 1800^{\circ} \mathrm{C}\right)[8]$.

Graphene has recently become a material of interest in supercapacitor application due to its high theoretical surface area and electrical conductivity [11-14]. Miller and 
coworkers [11] demonstrated that vertically oriented graphene grown on nickel foam has excellent frequency response with an RC time constant of less than $0.2 \mathrm{~ms}$. However, this was achieved at the cost of low specific capacitance resulting from the low density of electrode materials [11]. The performance of graphene-based supercapacitor materials is usually hindered by the fact that graphene sheets tend to aggregate and restack during processing and the actual accessible surface area of the electrodes is much lower compared to the theoretical surface area (more than $2600 \mathrm{~m}^{2} \mathrm{~g}^{-1}$ ). One of the effective strategies to avoid this problem is the addition of spacers such as carbon nanotubes (CNTs) between graphene sheets to prevent their restacking [15-17]. Another interesting approach to increase the accessibility of electrolyte ions to graphene sheets is the approach reported by Yoo and coworkers [18] where the in-plane design of the two supercapacitor electrodes resulted in a dramatic increase in capacitance compared to the conventional 2D stacking of the electrodes. The authors suggested that the in-plane design will increase the accessibility of ions to the surface of the graphene sheets and thus improve the capacitive properties [18]. It can be anticipated that the efficiency of the in-plane design of the electrode can be increased if the electrodes were made in micrometer scale sizes. There have been some efforts to utilize graphene as electrode material for micro-supercapacitors. Gao et al. [10] reported a direct write process to fabricate micro-supercapacitors with interdigital electrode design and hydrated graphene oxide (GO) as the electrolyte and separator. While the fabrication method is promising, the electrodes demonstrated in this work was fabricated in millimeter scales with the maximum capacitance of only $0.51 \mathrm{mFcm}^{-2}$ for an in-plane design of electrodes. It 
remains a challenge to develop micron-size patterned graphene electrodes through a scalable and reliable fabrication method.

In this work we report on ultra-high power micro-supercapacitors based on binder-free reduced graphene oxide ( $\mathrm{rGO}$ ) and $\mathrm{rGO} / \mathrm{CNT}$ hybrid as electrode materials. The micro-supercapacitors with interdigital microelectrodes $(100 \mu \mathrm{m}$ width and $50 \mu \mathrm{m}$ spacing) are readily fabricated through the combination of electrostatic spray deposition (ESD) and photolithography lift-off. We first demonstrate that ESD can be used for simultaneous deposition and reduction of GO. Next, in order to maximize the accessibility of electrolyte ions to electrode materials, we demonstrate the effects of the addition of CNTs between rGO sheets when integrated in micron-sized in-plane electrodes. The electrochemical properties of micro-supercapacitors were examined by cyclic voltammetry (CV), galvanostatic charge-discharge (CD), and electrochemical impedance spectroscopy (EIS). The micro-supercapacitors show exceptionally high rate capability and power handling performance and can be charged and discharged at $\mathrm{CV}$ scan rate of $50 \mathrm{Vs}^{-1}$ and $\mathrm{CD}$ rate of $100 \mathrm{mAcm}^{-2}\left(\sim 450-600 \mathrm{Ag}^{-1}\right)$. These rates are about three orders of magnitude higher than the charge and discharge rates of conventional supercapacitors. EIS measurements show very high frequency response of the microsupercapacitors with characteristic frequencies as high as $290.76 \mathrm{~Hz}$, higher than the recently reported state of the art micro-supercapacitors.

\subsection{Experimental Section}

\section{$\underline{\text { Preparation of interdigital microelectrodes and removable mask }}$}

First, a Ti $(20 \mathrm{~nm}) / \mathrm{Au}(300 \mathrm{~nm})$ layer was formed on a $\mathrm{Si} /(500 \mathrm{~nm}) \mathrm{SiO}_{2}$ by an electron-beam evaporation system. The interdigital current collectors were made by 
conventional photolithography and wet etching of the Ti/Au layer. To avoid deposition of materials in the space between the microelectrodes and on the contact pads, a removable mask was made on the samples by photolithography. The mask had two layers, a thin $(\sim 20 \mathrm{~nm})$ Omnicoat sacrificial bottom layer and a thick $(\sim 12 \mu \mathrm{m})$ SU-8 (Microchem, USA) top layer. First, the Omnicoat was spin coated on the substrate and was baked at $200{ }^{\circ} \mathrm{C}$ for 60 s. Then the SU-8 layer was spin coated, and then baked for $180 \mathrm{~s}$ at $65^{\circ} \mathrm{C}$ and $300 \mathrm{~s}$ at $95^{\circ} \mathrm{C}$. The mask was patterned by photolithography with an OAI 800 mask aligner (OAI, USA) to uncover the microelectrodes. The excess Omnicoat on the uncovered parts of micro-electrodes was removed by oxygen plasma treatment at 200 mTorr with a power of $100 \mathrm{~W}$ for $30 \mathrm{~s}$.

\section{Preparation of precursor solutions}

Single layer GO (6 mg, 0.7-1.2 $\mathrm{nm}$ thickness and 300-800 $\mathrm{nm}$ dimension, Cheaptubes, Inc., USA) was added to 1,2-propanediol (20 ml, sigma-Aldrich, USA) and dispersed by sonication for $30 \mathrm{~min}$ with an ultrasonic probe $(750 \mathrm{~W}, 20 \mathrm{KHz}$, Sonics and materials Inc., USA) to form a $0.3 \mathrm{mgml}^{-1} \mathrm{GO}$ solution. This solution was directly used in ESD deposition of rGO samples. In the case of GO-CNT solutions, appropriate amounts of $\mathrm{COOH}$-functionalized multiwalled CNT (8-15 nm diameter and 10-50 $\mu \mathrm{m}$ length Cheaptubes, Inc, USA) was added to the above solution to form solutions with GO:CNT ratios of $9: 1$ and $8: 2$, respectively.

\section{Electrostatic Spray deposition}

All the samples were deposited by the ESD for $2 \mathrm{~h}$ on the masked Ti/Au interdigital microelectrodes. The samples were preheated to $250^{\circ} \mathrm{C}$ before the deposition. The prepared precursor solutions were fed to a stainless steel needle using a syringe 
pump at the rate of $4-5 \mathrm{mlh}^{-1}$. The distance between the needle and the substrate was kept at $4 \mathrm{~cm}$. The solution was sprayed onto the substrate by applying a voltage of $6-7 \mathrm{kV}$ to the needle. After the deposition, the samples were soaked in remover PG (Microchem, USA) to remove the SU8 mask by etching the Omnicoat sacrificial layer.

\section{Characterization of samples}

The morphologies of as prepared samples were investigated using a JEOL 7000 field-emission scanning electron microscope (FE-SEM, JEOL, Japan). In order to study the reduction of GO during deposition, FTIR (JASCO FT/IR 4100 spectrometer) was used to analyze the oxygen functionalities of the sample GO before and after deposition. XPS (Physical Electronics 5400 ESCA) was used to quantitatively analyze the chemical compositions of GO and the resulting rGO after the deposition.

\section{Electrochemical testing}

After the fabrication of the micro-supercapacitors, the contact pads of each microdevice was connected to aluminum foil using silver paste. Then the microdevices were placed in a homemade Teflon cell with sealed cavity for the electrolyte. After filling the cavity of the cell with $3 \mathrm{M} \mathrm{KCL}$ electrolyte, electrochemical studies were performed using a VMP3 multichannel potentiostat (VMP3, Bio-Logic, USA) in the two-electrode mode and at room temperature. CVs were performed at scan rates ranging from 0.01 to $50 \mathrm{Vs}^{-1}$ in a potential range from 0 to $1 \mathrm{~V}$. CD measurements were carried out in the same potential window and with current densities ranging from 3 to $100 \mathrm{mAcm}^{-2}$. EIS measurements were performed at open circuit voltage (OCV) by applying a sinusoidal signal of $10 \mathrm{mV}$ amplitude at frequencies ranging from $100 \mathrm{kHz}$ to $50 \mathrm{mHz}$. The 
calculation of specific capacitance and energy and power densities from the above tests are described in the supporting information.

\subsection{Results and Discussions}

A homogeneous and stable solution of GO in 1,2 propanediol was used as the precursor solution for ESD deposition of GO on preheated $\left(250^{\circ} \mathrm{C}\right)$ substrates. The studies of the surface chemistry of deposited films with Fourier Transform Infrared Spectroscopy (FTIR) and X-ray photoelectron spectroscopy (XPS) show that the GO reduced to rGO during the deposition. The FTIR Spectra of GO before and after deposition are shown in Figure 6.1. The broad adsorption peak centering at around 3300 $\mathrm{cm}^{-1}$ in the spectrum of GO is assigned as isolated hydroxyl groups and water, which also signals an $\mathrm{H}-\mathrm{O}-\mathrm{H}$ bending at $1635 \mathrm{~cm}^{-1}[19,20]$. The peak at $1054 \mathrm{~cm}^{-1}$ is consistent with C-O stretching vibration. The presence of phenol and carboxylic acid groups was signaled by the phenolic $\mathrm{C}-\mathrm{O}$ peak at $1222 \mathrm{~cm}^{-1}$ and the mode at $1726 \mathrm{~cm}^{-1}$ which is assigned to $\mathrm{C}=\mathrm{O}$ stretching vibrations from carbonyl and carboxylic groups. Based on the structural model of GO, these groups are placed on the periphery of GO sheets [20,21]. The spectrum also shows the presence of epoxy C-O $\left(970 \mathrm{~cm}^{-1}\right)$ and $\mathrm{O}-\mathrm{H}$ bending in tertiary alcohol $\left(1361 \mathrm{~cm}^{-1}\right)$ groups which are reported to be located on the basal plane of GO [20,21]. After the deposition, the intensities of modes from water and oxygen functionalities were significantly reduced. The FTIR spectrum of deposited rGO shows mainly bands originating from $\mathrm{C}-\mathrm{O}$ stretching, phenolic $\mathrm{C}-\mathrm{O}$ stretching and $\mathrm{C}=\mathrm{O}$ stretching while the signals from tertiary alcohol and epoxy C-O on the basal plane of GO disappeared after deposition. The FTIR analysis suggests that the remaining oxygen 
groups after reduction are the functional groups that are mainly attached to the periphery of rGO sheets $[20,21]$.

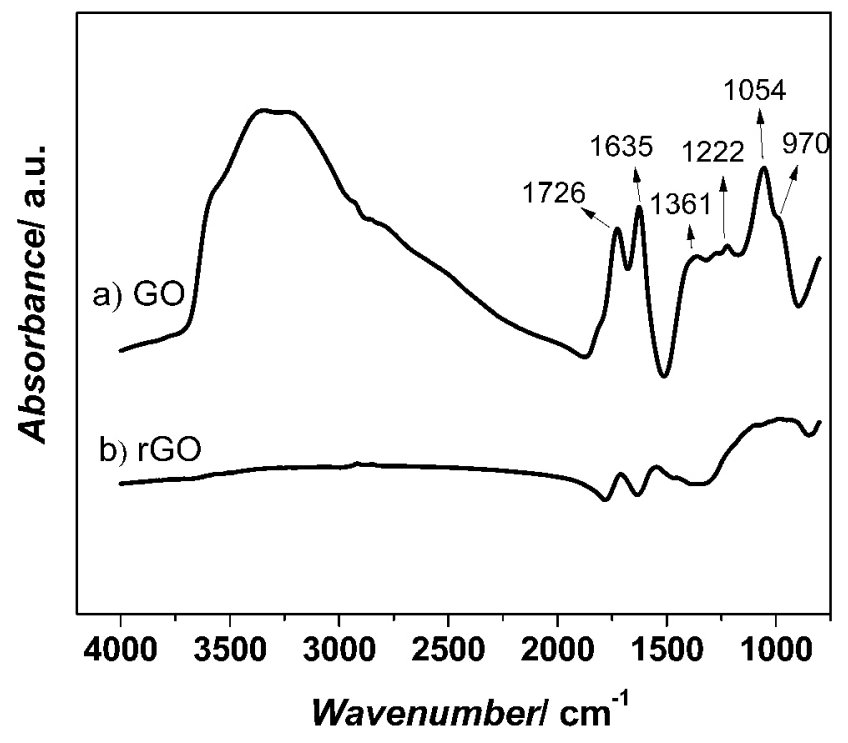

Figure 6.1 FTIR absorbance spectra of (a) as-purchased GO powder and (b) ESD deposited rGO.

The surface chemistry of GO and the as-deposited rGO were studied by X-ray photoelectron spectroscopy (XPS). The XPS spectra of GO and rGO are shown in Figure $6.2 \mathrm{a}$ and $\mathrm{b}$. The $\mathrm{O} 1 \mathrm{~s}$ peak intensity has decreased for deposited $\mathrm{rGO}$ and the overall $\mathrm{C} / \mathrm{O}$ ratio has increased to 5.75 compared to 1.77 for the GO powder. This seemingly mediocre increase in $\mathrm{C} / \mathrm{O}$ ratio represents a large decrease in oxygen content $(\sim 70 \%)$ on the surface rGO sheets. The $\mathrm{C} 1 \mathrm{~s}$ spectrum of $\mathrm{GO}$ can be deconvoluted to four components corresponding to four types of carbon bonds within GO. The peaks centered at 284.6, 286.6, 288.1 and $289.7 \mathrm{eV}$, correspond to $\mathrm{C}-\mathrm{C}$ in aromatic rings, C-O (epoxy and alkoxy), $\mathrm{C}=\mathrm{O}$ (carbonyl and carboxylic) and $\mathrm{COOH}$ groups, respectively [19,20,22]. The C1s spectrum of rGO shows all these four peaks with different proportions. The proportion of $\mathrm{C}-\mathrm{C}$ bond has increased from $50 \%$ for $\mathrm{GO}$ to about $61 \%$ for deposited rGO. 

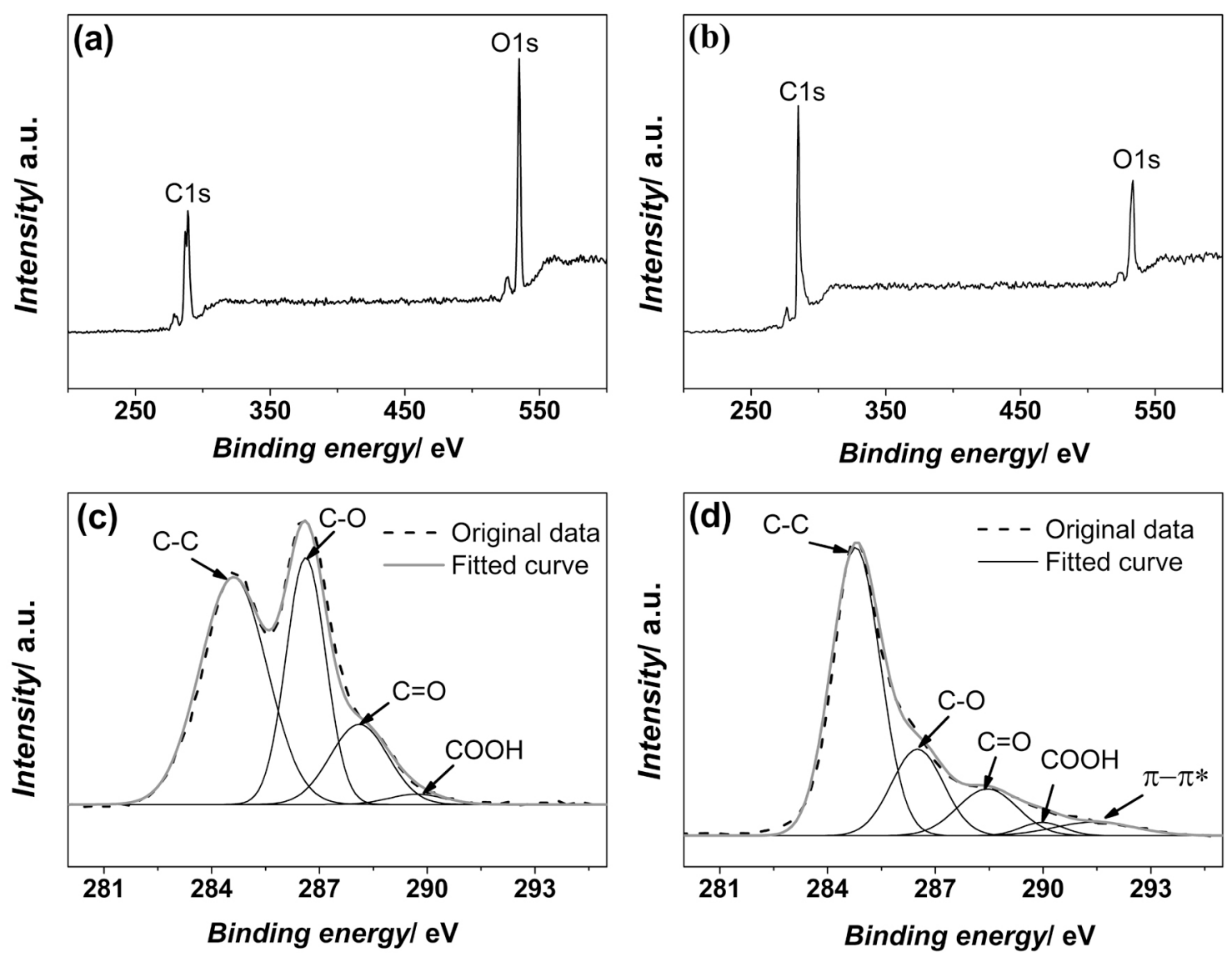

Figure 6.2 Wide-range XPS spectra of (a) GO and (b) rGO. The C1s spectra were deconvoluted into their corresponding components using a Gaussian function for (c) GO and (d) rGO.

The proportion of the peak corresponding to $\mathrm{C}-\mathrm{O}$ groups $(286.6 \mathrm{eV})$ has decreased after the deposition. The proportion of $\mathrm{C}-\mathrm{O}$ groups is $32 \%$ for $\mathrm{GO}$ and about $19 \%$ for the rGO. The remaining C-O groups should correspond to peripheral phenolic and carboxyl functionalities. The proportion of the $\mathrm{C}=\mathrm{O}$ groups shows a slight decrease after deposition, from $15 \%$ for $\mathrm{GO}$ to $12 \%$ for rGO. In addition to these four peaks, a $\pi$ $\pi^{*}$ shake up satellite peak was observed for $\mathrm{rGO}$ at around $291.4 \mathrm{eV}$. This is a characteristic of aromatic or conjugated systems which indicates that there are less 
defects in the structure of rGO after the deposition $[22,23]$. The XPS results confirm the FTIR results and show the reduction of GO after the ESD deposition. This is in agreement with previous studies regarding the reduction of GO at low temperatures (150$250^{\circ} \mathrm{C}$ ) in air or in organic solvents $[19,24]$. The remaining oxygen content after the reduction should correspond to phenolic, carbonyl, and carboxyl groups in the periphery of deposited rGO sheets [20].

Figure $6.3 \mathrm{a}$ schematically shows the procedure used to integrate electrode materials on interdigital Ti/Au microelectrodes to fabricate micro-supercapacitors. Before the ESD deposition, the working area of the microelectrodes was defined by a removable microfabricated photoresist mask that covers the contact pads and the space between the microelectrodes. After the deposition and removal of the mask, a micro-supercapacitor with 20 in-plane interdigital microelectrodes $(10$ positive and 10 negative microelectrodes) was constructed. Each microelectrode was $100 \mu \mathrm{m}$ in width and 2500 $\mu \mathrm{m}$ in length and the distance between adjacent microelectrodes were $50 \mu \mathrm{m}$. The samples labeled as rGO and CNT were fabricated from the deposition solution containing $100 \% \mathrm{GO}$ and $100 \% \mathrm{CNT}$, respectively. The samples labeled as rGO-CNT-9-1 and rGOCNT-8-2 had GO:CNT weight ratios of 9:1 and 8:2, respectively. The thicknesses of all electrodes were kept around $6 \mu \mathrm{m}$ by adjusting the deposition rate for each type of electrode materials. Figures $6.3 \mathrm{~b}$ and $\mathrm{c}$ show the scanning electron microscopy (SEM) micrographs of a typical fabricated micro-supercapacitor. The microelectrodes had welldefined and defect-free patterns and no short circuit between the electrodes was detected. 

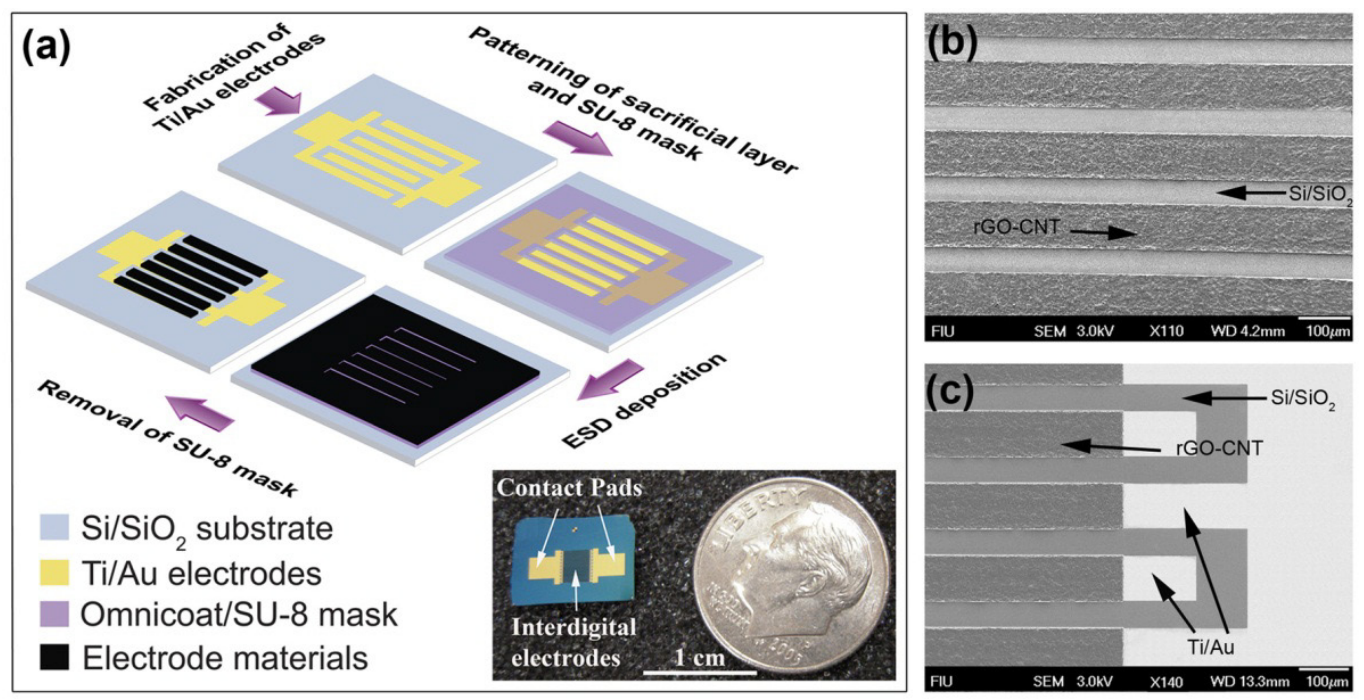

Figure 6.3 (a) Schematic drawing of fabrication procedures of micro-supercapacitors (inset shows a digital photograph of a fabricated device). (b) and (c) top view SEM micrographs of rGO-CNT based interdigital microelectrode arrays.

The microstructure of deposited rGO microelectrodes (Figure 4a) showed stacked layers of graphene sheets with micron-sized wrinkles that are probably the result of GO sheets bending during the deposition. Figure $6.4 \mathrm{~b}$, a tilted view from the side of an interdigital electrode, shows the local folding and non-uniform stacking of the rGO layers. Several to hundreds of stacked graphene sheets can be observed locally with extended irregular porous structures, which could act as diffusion channels and facilitate easy penetration of ions in the bulk of the microelectrodes. However, heavily stacked rGO sheets could prevent the full access of electrolyte ions to the surface rGO sheets. Figures $6.4 \mathrm{c}$ to $\mathrm{f}$ show the SEM images of rGO-CNT hybrid electrodes. The tilted view SEM images clearly show uniformly packed film with the appearance of CNTs between the rGO sheets throughout the thickness of deposited films with almost no sign of stacked rGO sheets. 

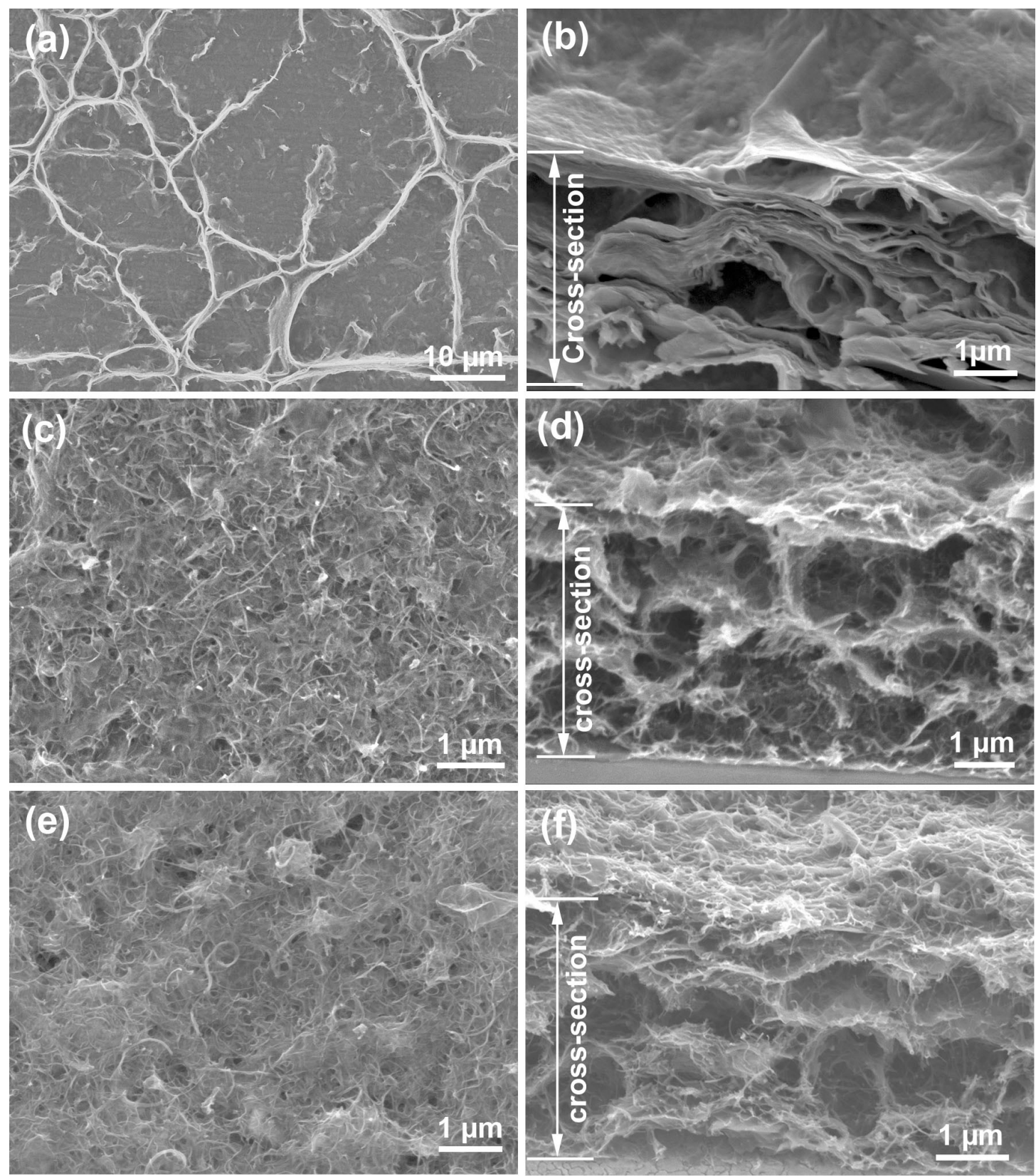

Figure 6.4 Scanning electron micrographs showing the morphology of the deposited electrodes:

Top view and tilted $35^{\circ}$ view of (a) and (b) rGO micro-electrodes, (c) and (d) rGO-CNT-9-1 micro-electrodes, and (e) and (f) rGO-CNT-8-2 microelectrodes. 
In order to study the electrochemical performance of fabricated microsupercapacitors, $\mathrm{CV}$ was conducted in $3 \mathrm{M} \mathrm{KCl}$ aqueous electrolyte and at the potential range of 0 to $1 \mathrm{~V}$. During the initial $\mathrm{CV}$ cycles of the rGO micro-supercapacitor at a 0.1 $\mathrm{Vs}^{-1}$ scan rate, the $\mathrm{CV}$ current during cycling constantly increased up to 200 cycles, with the average current density increasing by more than 7 times compared to the first cycle (Figure 6.5a). After about 200 cycles the rate of the increase in average current dropped and for the following cycles up to the $1000^{\text {th }}$ cycle, an increase of less than $5 \%$ in current density was noted (Figures $6.5 \mathrm{a}$ and $\mathrm{b}$ ). A similar phenomenon has been reported by Cheng et al. during long time cycling of graphene electrodes and was referred to as "electro-activation" [15]. The authors detected a 60\% increase in specific capacitance of pristine graphene electrodes during longtime cycling. It was suggested that the intercalation of electrolyte ions between the graphene sheets increases the spacing between the sheets and therefore increases the accessibility of ions to the surface of graphene. It should be noted that the effect of electro-activation is much more pronounced in the case of the interdigital rGO electrodes compared to the planar electrodes reported by Cheng and coworkers [15]. In general, the in-plane design of the graphene electrodes increases the accessibility of ions in between graphene electrodes [18]. In the case of rGO micro-supercapacitors, in addition to the side by side design of the electrodes, the smaller size of the electrode and the shorter distance between them further facilitate the accessibility of ions to the graphene sheets, resulting in a more efficient electro-activation. In contrast to the $\mathrm{rGO}$ microdevice, the average $\mathrm{CV}$ current density of rGO-CNT micro-supercapacitors did not increase during the cyclic test for 
1000 cycles showing that the electro-activation did not occur in the case of these microdevices (Figure 6.5b).
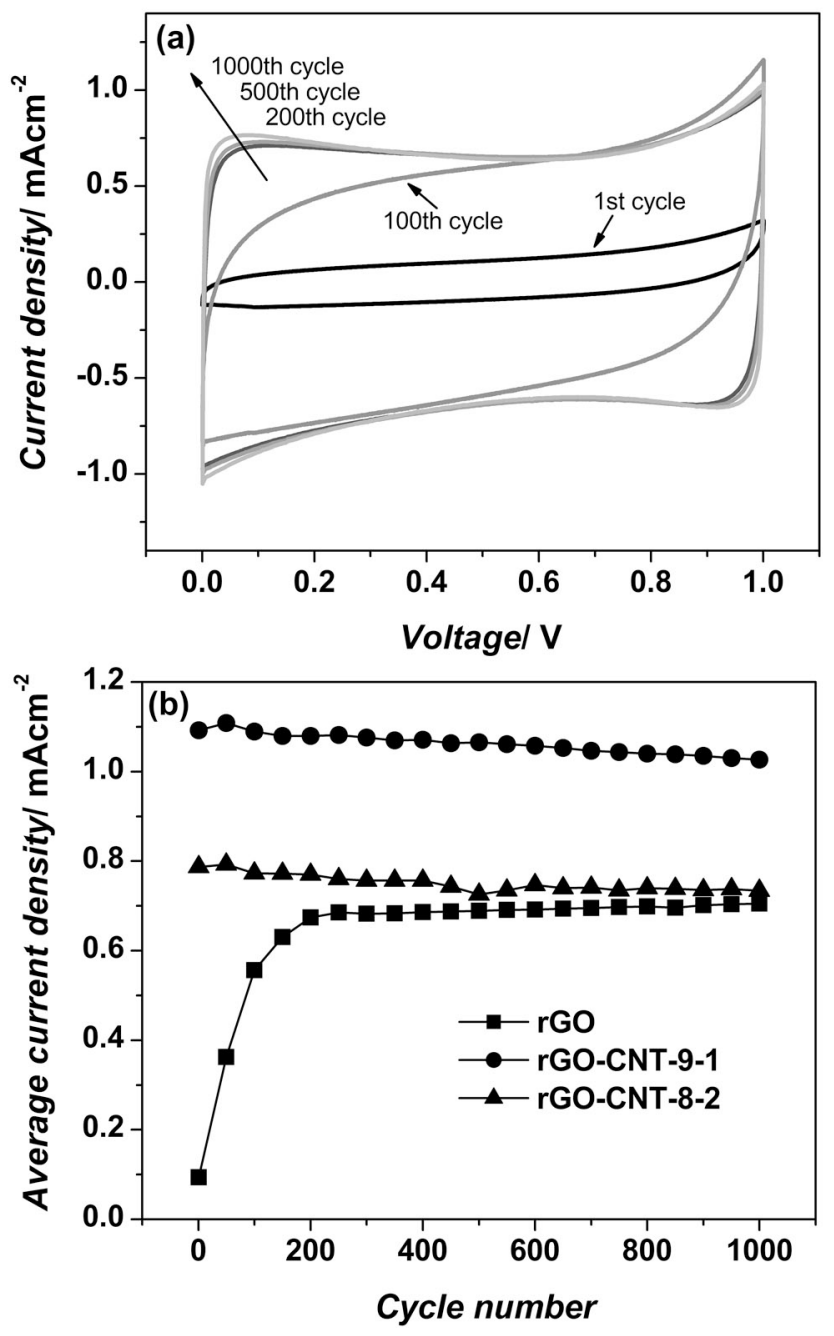

Figure 6.5 (a) CV curves at various cycles of a rGO micro-supercapacitors tested at $0.1 \mathrm{Vs}^{-1}$ scan rate. (b) Variation of average CV current density with cycle number for rGO, rGO-CNT-9-1 and rGO-CNT-8-2.

This observation leads us to conclude that the addition of CNTs had effectively prevented the restacking of rGO sheets and thus the intercalation of ions during cycling could not further increase the spacing between the rGO sheets. Furthermore, the 
microdevices with hybrid rGO-CNT electrodes show higher $\mathrm{CV}$ current density, implying that compared to electro-activation, using CNTs as a spacer between graphene sheets is a more effective way to increase the accessible surface area of the electrodes. Figure 6.6 schematically shows the two mechanism of increase in accessibility of electrodes to the electrolyte ions that were explained above.

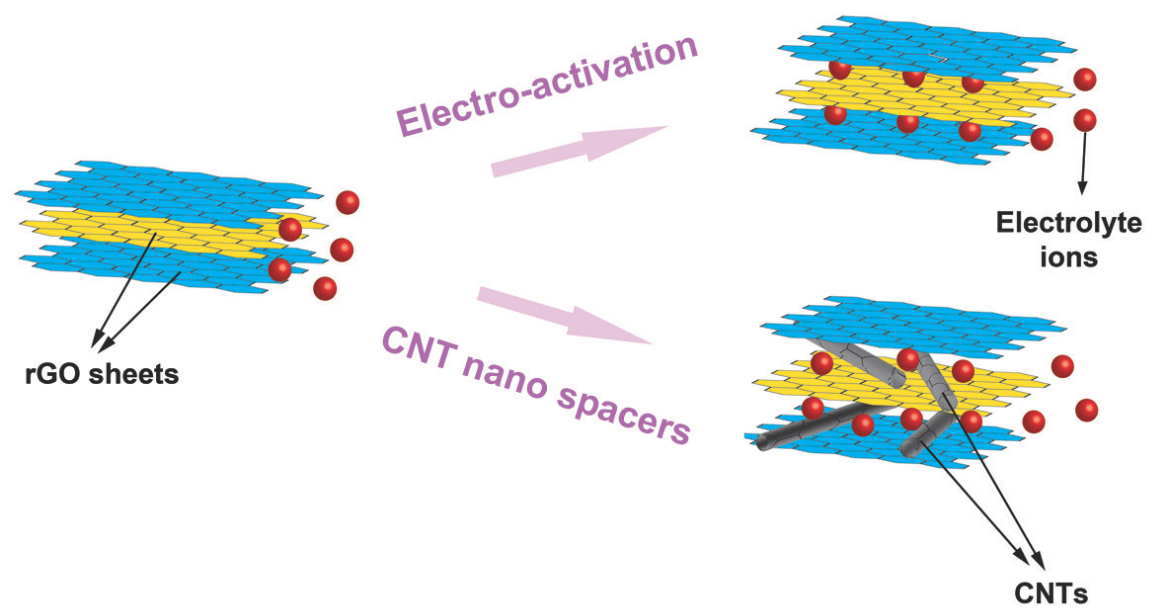

Figure 6.6 Schematic drawing showing the two mechanisms of increasing the ion accessibility of electrodes.

The rate capability and power handling of the micro-supercapacitors was tested by $\mathrm{CV}$ at very high scan rates $\left(1\right.$ to $\left.50 \mathrm{Vs}^{-1}\right)$. Before performing the tests at higher scan rates, each microdevice was cycled for 250 cycles at $0.1 \mathrm{Vs}^{-1}$ to ensure that the $\mathrm{CVs}$ sere stable and in the case of the rGO microdevices the electro-activation was completed. Figures 6.7 to 6.11 show the $\mathrm{CV}$ curves of rGO, rGO-CNT-9-1 and rGO-CNT-8-2 microdevices at different scan rates. The rGO micro-supercapacitor showed near rectangular CV curves, which is typical of EDLCs, at scan rates of 1 and $5 \mathrm{Vs}^{-1}$. Upon increasing the scan rate to $10 \mathrm{Vs}^{-1}$, the $\mathrm{CV}$ curve deviated from a rectangular shape which 
indicates a more resistive behavior. At much higher scan rates of 25 and $50 \mathrm{Vs}^{-1}$, the rGO microdevice still showed some capacitive behavior, but the resistive behavior was dominant and the capacitance dropped quickly at these scan rates. In the case of microdevices with rGO-CNT electrodes, the $\mathrm{CV}$ curve showed a rectangular shape with pure capacitive behavior even at a very high scan rate of $50 \mathrm{Vs}^{-1}$. The stack capacitance (volumetric capacitance) of the micro-supercapacitors were calculated from the $\mathrm{CV}$ curves at various scan rates and by taking into account the volume of both electrodes and the space between them (Figure 6.12). As suggested by Gogotsi and Simon [25], the volumetric or areal capacitance or energy density are much more reliable performance metrics for supercapacitor devices compared to gravimetric capacitance. This is more pronounced in the case of microdevices as the weight of the material of a thin film electrode on a chip is negligible [25].

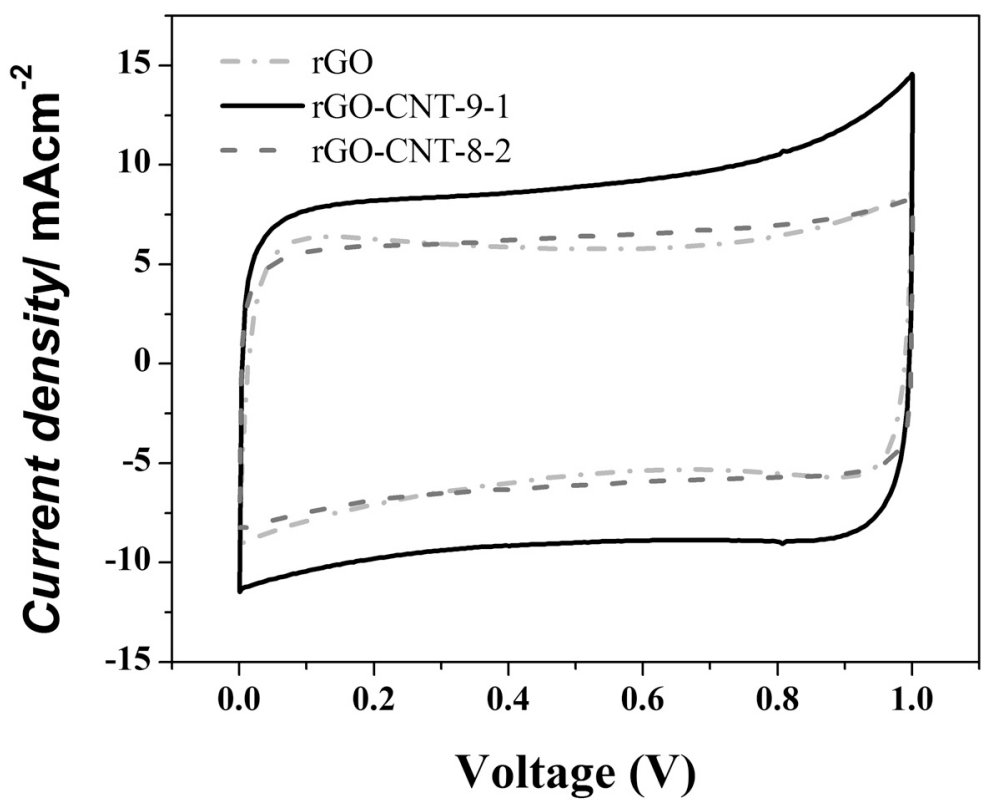

Figure 6.7 CV curves of rGO, rGO-CNT-9-1 and rGO-CNT-8-2 micro-supercapacitors at $1 \mathrm{Vs}^{-1}$ scan rate. 


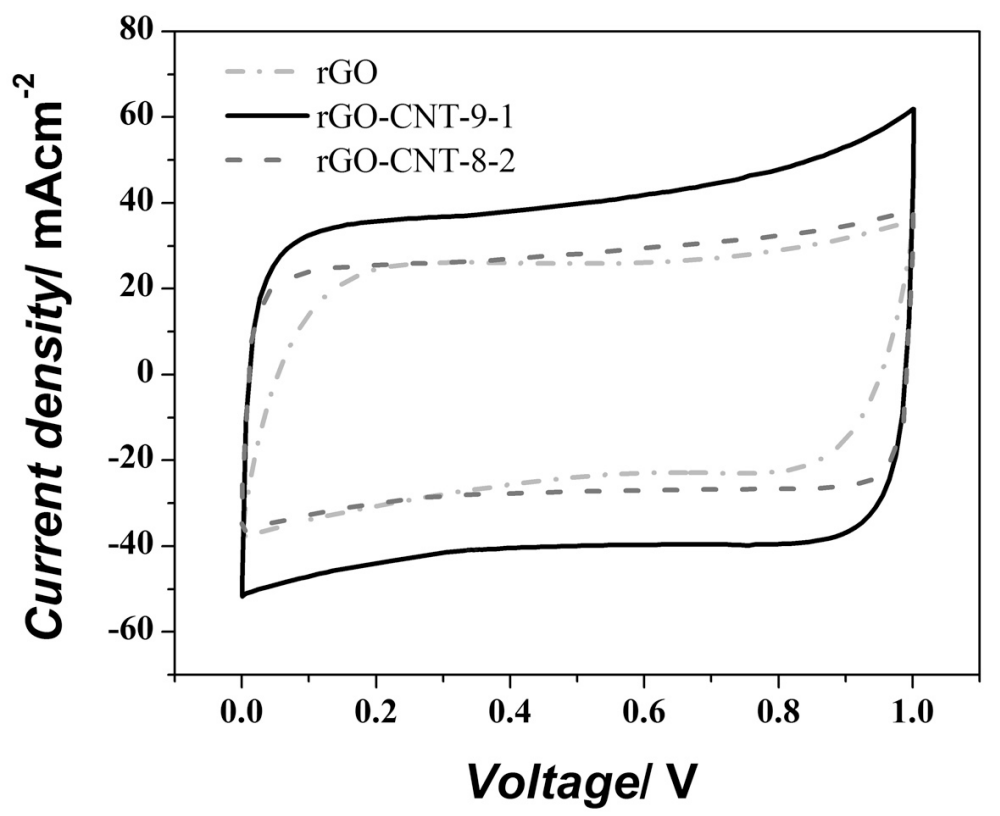

Figure 6.8 CV curves of rGO, rGO-CNT-9-1 and rGO-CNT-8-2 micro-supercapacitors at $5 \mathrm{Vs}^{-1}$ scan rate.

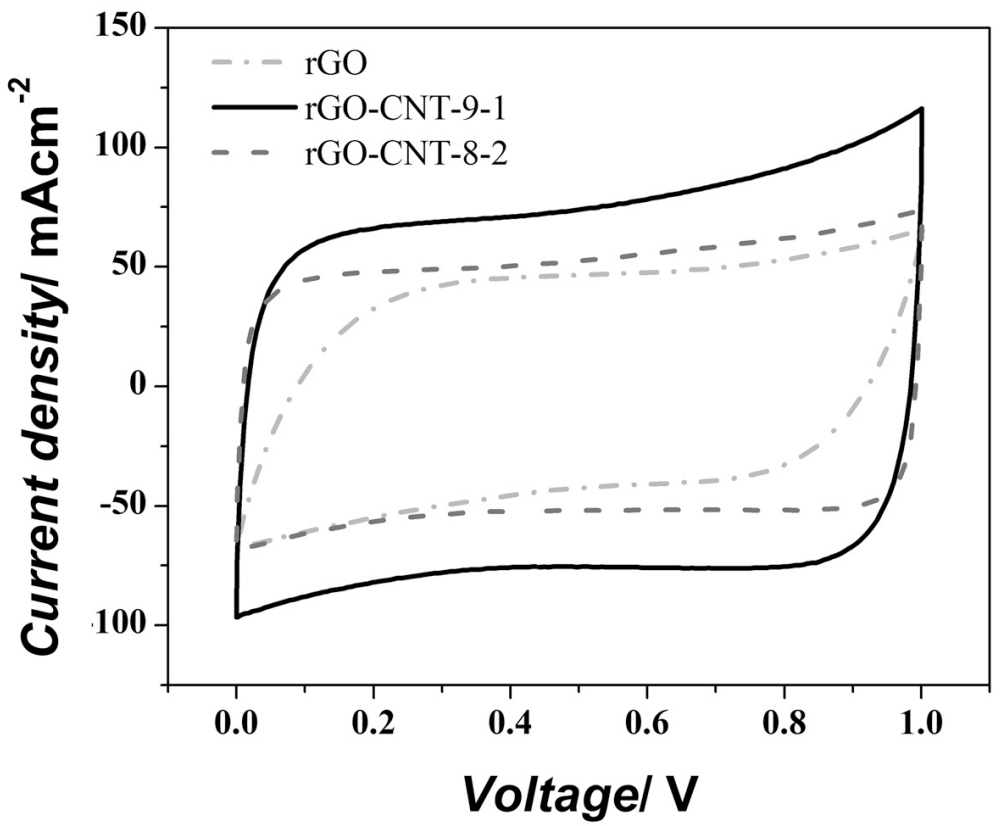

Figure 6.9 CV curves of rGO, rGO-CNT-9-1 and rGO-CNT-8-2 micro-supercapacitors at $10 \mathrm{Vs}^{-1}$ scan rate. 


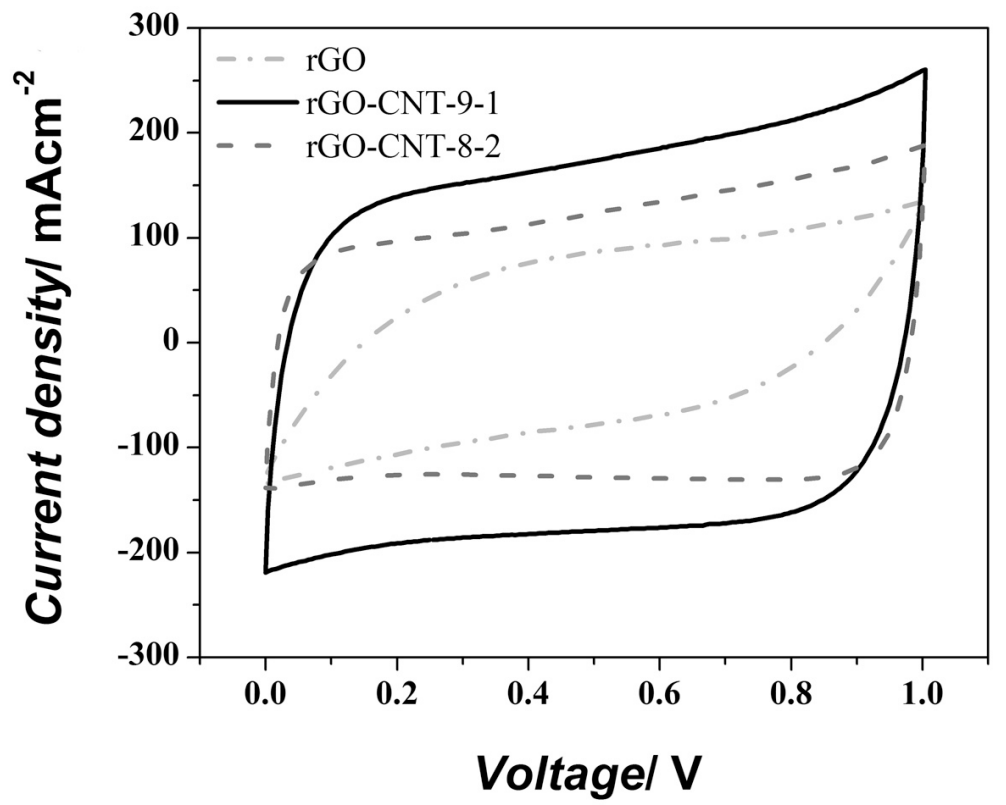

Figure 6.10 CV curves of rGO, rGO-CNT-9-1 and rGO-CNT-8-2 micro-supercapacitors at 25 $\mathrm{Vs}^{-1}$ scan rate.

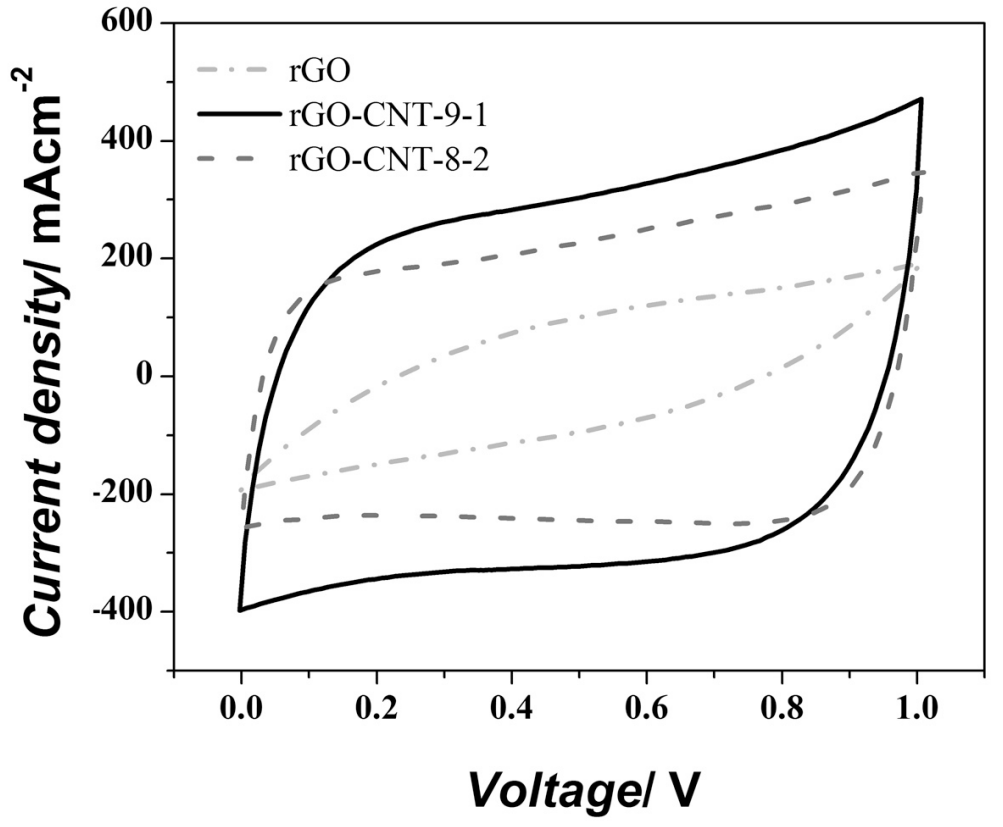

Figure 6.11 CV curves of rGO, rGO-CNT-9-1 and rGO-CNT-8-2 micro-supercapacitors at 50 $\mathrm{Vs}^{-1}$ scan rate. 


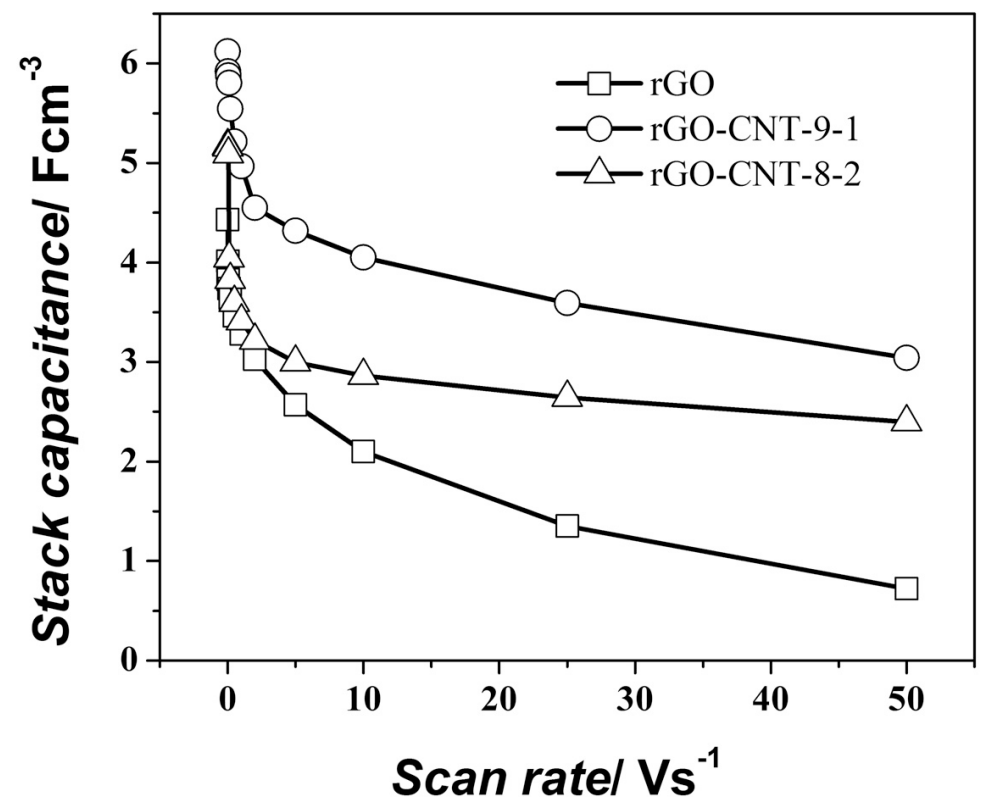

Figure 6.12 Comparison of stack capacitances of micro-supercapacitors with different electrode compositions.

As it is evident from Figure 6.12, all micro-supercapacitors with different compositions showed capacitive behavior even at a very high scan rate of $50 \mathrm{Vs}^{-1}$. However, the rGO-CNT micro-supercapacitors showed better performance in terms of capacitance and rate capability. The rGO micro-supercapacitor showed a stack capacitance of about $4.4 \mathrm{Fcm}^{-3}\left(\sim 27.2 \mathrm{Fcm}^{-3}\right.$ volumetric capacitance of one electrode) at a scan rate of $0.01 \mathrm{Vs}^{-1}$ which dropped to $3.2 \mathrm{Fcm}^{-3}$ at a $1 \mathrm{Vs}^{-1}$ scan rate. At a higher scan rate of $50 \mathrm{Vs}^{-1}$, the stack capacitance decreased to $0.7 \mathrm{Fcm}^{-3}\left(\sim 4.4 \mathrm{Fcm}^{-3}\right.$ volumetric capacitance of one electrode). This value at a $50 \mathrm{Vs}^{-1}$ scan rate is about $16 \%$ of the initial stack capacitance recorded at a $0.01 \mathrm{Vs}^{-1}$ scan rate. The rGO-CNT-9-1 microsupercapacitor showed the highest stack capacitance at all CV scan rates. At the low scan rate of $0.01 \mathrm{Vs}^{-1}$, the stack capacitance was about $6.1 \mathrm{Fcm}^{-3}$ which is equivalent to 37.5 $\mathrm{Fcm}^{-3}$ volumetric capacitance of one electrode. When the scan rate was increased to $1 \mathrm{Vs}^{-}$ 
${ }^{1}$ the stack capacitance dropped to about $5.0 \mathrm{Fcm}^{-3}$. At the scan rate of $50 \mathrm{Vs}^{-1}$ the stack capacitance was about $3.1 \mathrm{Fcm}^{-3}$ which is $50 \%$ of its value at the $0.01 \mathrm{Vs}^{-1}$ scan rate and $62 \%$ percent of its value at the $1 \mathrm{Vs}^{-1}$ scan rate. The rGO-CNT-8-2 micro-supercapacitor showed a stack capacitance of about $2.4 \mathrm{Fcm}^{-3}$ at a scan rate of $50 \mathrm{Vs}^{-1}$ which is $46 \%$ of the capacitance at a $0.01 \mathrm{Vs}^{-1}$ scan rate $\left(5.2 \mathrm{Fcm}^{-3}\right)$ and $70 \%$ of the capacitance at the scan rate of $1 \mathrm{Vs}^{-1}\left(3.4 \mathrm{Fcm}^{-3}\right)$. The lower capacitance of rGO-CNT-8-2 micro-supercapacitors could be explained by the effect of additional CNT which has lower capacitance compared to rGO sheets (Figure 6.12). However, the lower drop in stack capacitance of rGO-CNT-8-2 micro-supercapacitors (30\%) compared to the rGO-CNT-9-1 microsupercapacitor (38\%) upon increasing the scan rate from 1 to $50 \mathrm{Vs}^{-1}$ indicates that additional CNT has improved the rate capability of the microdevice. The microsupercapacitor based on $100 \%$ CNT shows much lower capacitance compared to $\mathrm{rGO}$ and rGO-CNT micro-supercapacitors at all scan rates (Figure 6.12). The areal specific capacitance of the rGO-CNT-9-1 microdevice (calculated by taking into account the total area of both electrodes) is about $2.8 \mathrm{mFcm}^{-2}$ at a $50 \mathrm{Vs}^{-1}$ scan rate. The significance of the electrochemical properties of the rGO-CNT micro-supercapacitors can be further revealed when they are compared to the performance of other reported microsupercapacitors. Even at a high scan rate of $50 \mathrm{Vs}^{-1}$ the rGO-CNT micro-supercapacitors showed higher specific capacitance compared to the majority of reported EDLC microsupercapacitors (specific capacitance of $0.4-2 \mathrm{mFcm}^{-2}$ at very low $\mathrm{CV}$ scan rates of 0.01 to $\left.0.1 \mathrm{Vs}^{-1}\right)[5,9,10,26]$. Some other reported EDLC micro-supercapacitors such as graphene-cellulose paper supercapacitors and CDC micro-supercapacitors show higher specific capacitance but poor rate capability and frequency response [7,27]. Finally, the 
high power OLC based micro-supercapacitors reported by Pech et al.[8] shows similar high rate capability but has lower capacitance compared to rGO-CNT microsupercapacitors. For instance, a stack capacitance of about $0.9 \mathrm{Fcm}^{-3}$ was measured at a $50 \mathrm{Vs}^{-1}$ scan rate for OLC based micro-supercapacitors. The electrochemical performance of micro-supercapacitors was further studied by CD at different current densities and EIS. The CD curves showed triangular shapes, typical of EDLC supercapacitors, with a very low $i R$ drop even at a very high current density of $60 \mathrm{mAcm}^{-2}$ (Figure 6.13a). The $i R$ drop, the sudden voltage drop at the beginning of the CD discharge, is a measure of the overall resistance of the cell and since its value is proportional to discharge current, the small $i R$ drop of the micro-supercapacitors at a high discharge current indicates a very low cell resistance for all the tested micro-supercapacitors.

The $i R$ drop slightly decreased with an increase in the amount of CNTs in the electrodes and its value was $0.024,0.013$ and $0.010 \mathrm{~V}$ for rGO, rGO-CNT-9-1, and rGOCNT-8-2 micro-supercapacitors, respectively. The areal specific capacitances of the micro-supercapacitors were calculated from $\mathrm{CD}$ curves at different discharge current densities and are shown in Figure 6.13b. The results from CD experiments were in good agreement with the $\mathrm{CV}$ results in terms of specific capacitance and rate handling of micro-supercapacitors. The highest specific capacitances at all discharge currents were achieved for the rGO-CNT-9-1 micro-supercapacitor with a specific capacitance of 5.1 $\mathrm{mFcm}^{-2}$ at a $3 \mathrm{mAcm}^{-2}$ discharge current density which dropped only by about $30 \%$ at a very high current density of $100 \mathrm{mAcm}^{-2}\left(3.6 \mathrm{mFcm}^{-2}\right)$. In comparison, the rGO-CNT-8-2 micro-supercapacitor showed a lower specific capacitance $\left(3.4 \mathrm{mFcm}^{-2}\right.$ at $3 \mathrm{mAcm}^{-2}$ current density), but slightly improved rate handling as its specific capacitance dropped 
by $26 \%$ upon increasing the discharge current to $100 \mathrm{mAcm}^{-2}$. Based on our estimation of the weight of the electrode materials for each micro-supercapacitor $(\sim 4.5-6.2 \mu \mathrm{g})$, a discharge areal current density of $100 \mathrm{mAcm}^{-2}$ approximately corresponds to gravimetric current density in the range of 450 to $600 \mathrm{Ag}^{-1}$, which is more than two orders of magnitude higher than the discharge current densities that are usually used in testing supercapacitors [28].
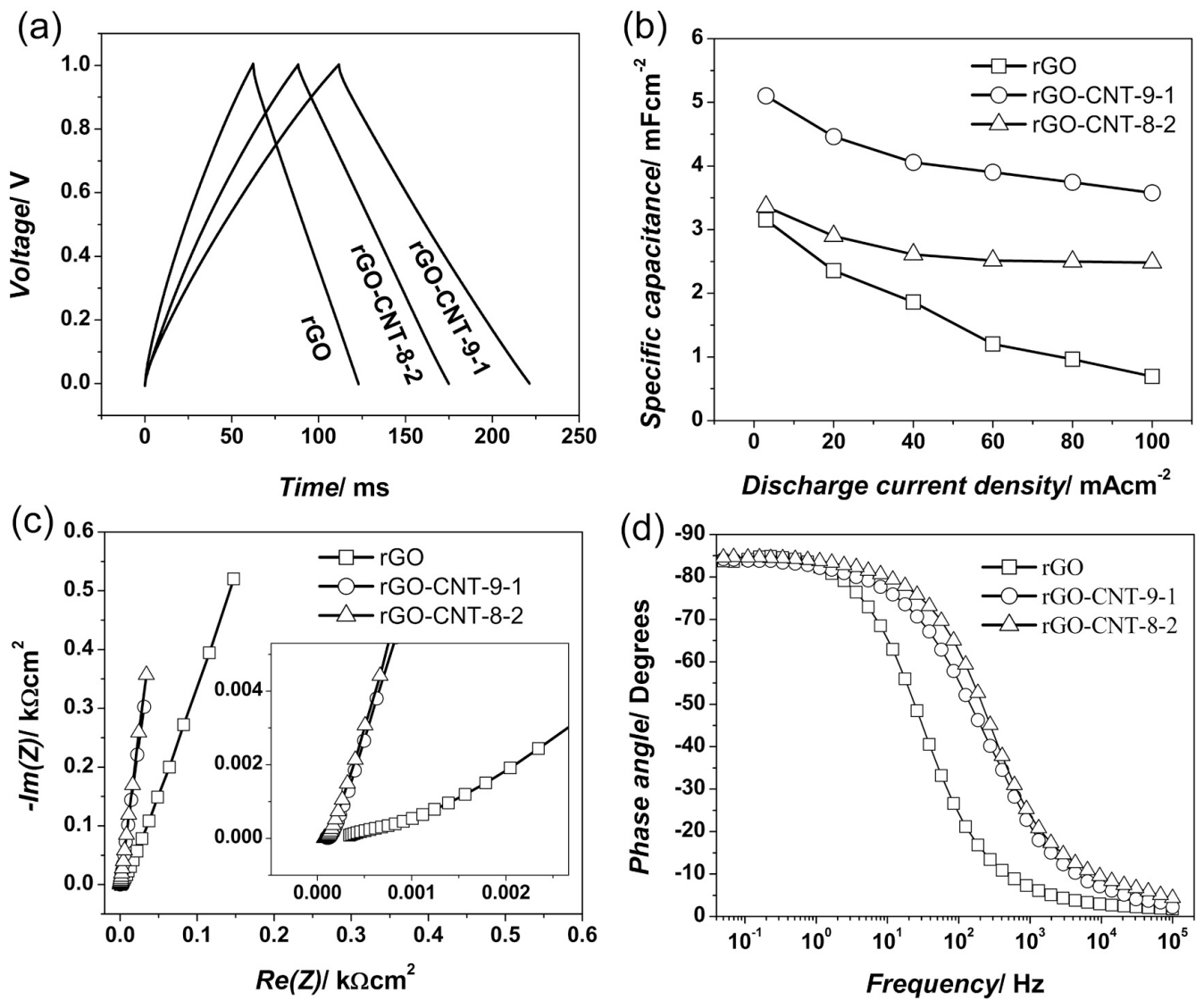

Figure 6.13 (a) Charge-discharge curves of micro-supercapacitors based on rGO, rGO-CNT-9-1 and rGO-CNT-8-2 electrodes. (b) Specific capacitances of micro-supercapacitor at different discharge current densities. (c) Nyquist plots of different micro-supercapacitors (inset shows the Nyquist plots at higher frequencies). (d) Phase angle vs. frequency for different microsupercapacitor. 
The frequency response of the micro-supercapacitors was studied by EIS. The Nyquist plots of the micro-supercapacitors show typical features of EDCL supercapacitors (Figure 6.13c). For an ideal EDLC the low-frequency region of the Nyquist plot is a straight line. The more vertical the line, the more closely the supercapacitor behaves as an ideal capacitor [12,13,29]. The low-frequency regions of Nyquist plots of rGO-CNT hybrid devices show straight lines with an almost $90^{\circ}$ angle. The slope of $45^{\circ}$ segment of a Nyquist plot is called the Warburg resistance and is a result of frequency dependence of diffusion of the electrolyte ions into the bulk of the electrode $[12,13]$. The much shorter Warburg regions of plots for rGO-CNT microsupercapacitors show the better diffusion of ions into the bulk of electrodes compared to the rGO micro-supercapacitors. Furthermore, the rGO-CNT microdevices show lower equivalent series resistance (ESR) compared to the rGO microdevice (ESR can be calculated from the X-intercepts of Nyquist plots).

For a more informative analysis of EIS tests, the dependence of phase angle with frequency of the microdevices was plotted in Figure 6.13d. The rGO microsupercapacitor showed frequency independent phase angles close to $-90^{\circ}$ for frequencies up to $2 \mathrm{~Hz}$ after which the phase angle increased with the increase in frequency. However, the rGO-CNT micro-supercapacitors showed frequency independent phase angle even at frequencies as high as $20 \mathrm{~Hz}$. A better comparison of the frequency response of the microdevices can be made by comparing their characteristic frequency $\left(\mathrm{f}_{0}\right)$ which is the frequency at a phase angle of $-45^{\circ}$ or its corresponding relaxation time constant $\left(\tau_{0}=1 / \mathrm{f}_{0}\right)$. The characteristic frequency marks the point at which the resistive and capacitive impedance are equal and at frequencies higher than $\mathrm{f}_{0}$ supercapacitor shows a 
more resistive behavior $[29,30]$. The corresponding relaxation time constant $\left(\tau_{0}\right)$ is the minimum time needed to discharge all the energy from the device with an efficiency of greater than $50 \%[8,29]$. The rGO-CNT-8-2 micro-supercapacitor showed the best frequency response with the characteristic frequency of about $290.7 \mathrm{~Hz}$ and time constant of $3.4 \mathrm{~ms}$. The rGO-CNT-9-1 micro-supercapacitor showed a slightly lower time constant of about $4.8 \mathrm{~ms}\left(\mathrm{f}_{0} \sim 208.6 \mathrm{~Hz}\right)$. In contrast, the time constant of rGO micro-supercapacitor was about $33 \mathrm{~ms}$. To the best of our knowledge, so far, the best frequency response for a micro-supercapacitor was reported by Pech et al.[8] for OLC micro-supercapacitor tested in $1 \mathrm{M} \mathrm{Et}_{4} \mathrm{NBF}_{4} /$ anhydrous propylene carbonate electrolyte. The OLC microsupercapacitor, however, showed a time constant of $26 \mathrm{~ms}$ and a modest specific capacitance of $1.7 \mathrm{mFcm}^{-2}$ at a $\mathrm{CV}$ scan rate of $1 \mathrm{Vs}^{-1}$, which are both improved in case of our micro-supercapacitors.

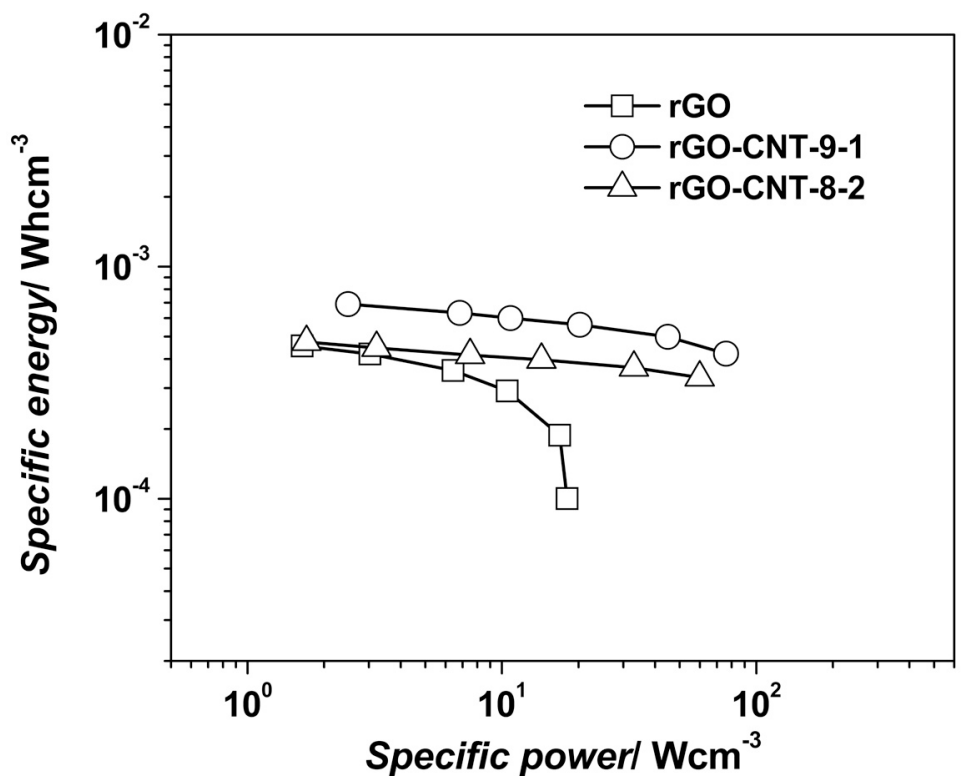

Figure 6.14 Ragone plot showing the relationship of specific energy and the specific power of micro-supercapacitors. 
The volumetric energy and power density of micro-supercapacitors were calculated from CVs at a scan rate of 1 to $50 \mathrm{Vs}^{-1}$ and shown in a Ragone plot (Figure 6.14). It is evident that the drop in energy density with increasing power density is very small in the case of rGO-CNT micro-supercapacitors. The highest energy density $(\sim 0.67$ $\left.\mathrm{mWhcm}{ }^{-3}\right)$ and the highest volumetric power density $\left(\sim 75 \mathrm{Wcm}^{-3}\right)$ were both achieved for the rGO-CNT-9-1 microdevices.

The high specific capacitance, exceptional rate capability and high frequency response of the rGO-CNT micro-supercapacitors can be explained by the synergic effects of electrode materials, method of electrode assembly and structural design of microsupercapacitors. First, using CNT as a nano spacer inhibits the agglomeration and restacking of graphene sheet, thus providing a highly accessible surface area for the microelectrodes. Second, the binder-free deposition based on the ESD technique plays a role in high power handling of the micro-supercapacitors. It is well known that the addition of polymeric binders that are typically used in the fabrication of the electrodes hinders their performance by increasing resistivity and the addition of dead weight $[11,30]$. Third, another important factor affecting the high power capability of our microsupercapacitors is the interdigital design of the electrodes. The small distance between the microelectrodes could minimize the electrolyte resistance by reducing the mean ionic diffusion pathway between the microelectrodes. Finally, the small size of the electrodes along with their side by side in-plane design facilitates the diffusion of electrolyte ions between the rGO sheets and in the entire thickness of the electrodes. The rGO-CNT micro-supercapacitors are able to satisfy the power needs of certain miniaturized electronic devices. For instance, they can power radio frequency identification (RFID) 
tags which generally require 1-100 $\mu \mathrm{W}$ [31]. Furthermore, the high frequency response of the rGO-CNT micro-supercapacitors makes them an ideal device to be coupled with other devices such as energy harvesters and micro-batteries to provide peak power. With further optimization of the electrode compositions and structural design of microsupercapacitors, they can potentially meet the necessary requirements to replace low energy and large electrolytic capacitors in ac line-filtering applications in portable electronics [11]. The reported fabrication method of micro-patterned rGO films can also be utilized for the development of other graphene based functional devices.

\subsection{Conclusions}

We have demonstrated the development of micro-supercapacitors based on rGO and rGO-CNT patterned microelectrodes with superior electrochemical properties through the combination of photolithography lift-off and ESD deposition. The fabrication process involved the ESD deposition of electrode materials on masked interdigital current collectors. The GO sheets in the precursor solution were readily reduced to rGO during the low temperature deposition, eliminating the need for further thermal or chemical reduction of GO. In the case of the rGO micro-supercapacitors, the diffusion of electrolyte ions between the rGO sheets resulted in electro-activation of the microelectrodes that increased the average $\mathrm{CV}$ current by more than 7 times during the first 200 cycles. We further demonstrated that the addition of CNTs as nano spacers between rGO sheets could minimize their restacking. The electrochemical performance tests indicated that while $\mathrm{rGO}$ microdevices had reasonable specific capacitance and power handling ability, the rGO-CNT micro-supercapacitor exhibited exceptional performance. The best results were achieved when a composition of $90 \% \mathrm{GO}$ and $10 \%$ 
CNT was used in the deposition solution. The stack capacitance of rGO-CNT-9-1 microsupercapacitors was about $5.0 \mathrm{Fcm}^{-3}$ at a $1 \mathrm{Vs}^{-1} \mathrm{CV}$ scan rate, which dropped only by $40 \%$ at a very high scan rate of $50 \mathrm{Vs}^{-1}$. The excellent power response of these microsupercapacitors was revealed by EIS experiments when an RC time constant of only 4.8 ms was measured at a $-45^{\circ}$ phase angle, which is lower than any other reported microsupercapacitors. Increasing the amount of CNTs to $20 \%$ slightly improved the power response and rate handling ability of the micro-supercapacitors, but had a negative impact on the specific capacitance. The developed micro-supercapacitors promise high energy micron-scale energy storage units that are able to provide enough energy and satisfy the peak power required for a number of applications. They can also potentially replace low energy electrolytic capacitors in miniaturized electronic devices. We anticipate that further improvement in the performance of micro-supercapacitors can be achieved through the optimization of ESD conditions, microelectrode design, and the composition of the electrode materials.

\subsection{References}

[1] M. Beidaghi, W. Chen, C. Wang, J. Power Sources 2011, 196, 2403.

[2] M. Xue, Z. Xie, L. Zhang, X. Ma, X. Wu, Y. Guo, W. Song, Z. Li, T. Cao, Nanoscale 2011, 3, 2703-8.

[3] M. Beidaghi, C. Wang, Electrochim. Acta 2011, 56, 9508.

[4] K. Wang, W. Zou, B. Quan, A. Yu, H. Wu, P. Jiang, Z. Wei, Adv. Energy Mater. 2011, 1, 1068-1072.

[5] D. Pech, M. Brunet, P.-L. Taberna, P. Simon, N. Fabre, F. Mesnilgrente, V. Conédéra, H. Durou, J. Power Sources 2010, 195, 1266.

[6] J. Chmiola, C. Largeot, P. L. Taberna, P. Simon, Y. Gogotsi, Science 2010, 328, 480 . 
[7] M. Heon, S. Lofland, J. Applegate, R. Nolte, E. Cortes, J. D. Hettinger, P.-L. Taberna, P. Simon, P. Huang, M. Brunet, Y. Gogotsi, Energy Environ. Sci. 2011, $4,135$.

[8] D. Pech, M. Brunet, H. Durou, P. Huang, V. Mochalin, Y. Gogotsi, P.-L. Taberna, P. Simon, Nat. Nanotechnol. 2010, 5, 651.

[9] M. Kaempgen, C. K. Chan, J. Ma, Y. Cui, G. Gruner, Nano let. 2009, 9, 1872.

[10] W. Gao, N. Singh, L. Song, Z. Liu, A. L. M. Reddy, L. Ci, R. Vajtai, Q. Zhang, B. Wei, P. M. Ajayan, Nat. Nanotechnol. 2011, 6, 6.

[11] J. R. Miller, R. a Outlaw, B. C. Holloway, Science 2010, 329, 1637.

[12] M. D. Stoller, S. Park, Y. Zhu, J. An, R. S. Ruoff, Nano lett. 2008, 8, 3498.

[13] Y. Wang, Z. Shi, Y. Huang, Y. Ma, C. Wang, M. Chen, Y. Chen, J. Phys. Chem. C 2009, 113, 13103.

[14] Y. Zhu, S. Murali, M. D. Stoller, K. J. Ganesh, W. Cai, P. J. Ferreira, A. Pirkle, R. M. Wallace, K. a Cychosz, M. Thommes, D. Su, E. A. Stach, R. S. Ruoff, Science 2011, 332, 1537.

[15] Q. Cheng, J. Tang, J. Ma, H. Zhang, N. Shinya, L.-C. Qin, Phys. Chem. Chem. Phys. 2011, 13, 17615.

[16] Y. Wang, Y. Wu, Y. Huang, F. Zhang, X. Yang, Y. Ma, Y. Chen, J. Phys. Chem. C 2011, 115, 23192.

[17] S.-Y. Yang, K.-H. Chang, H.-W. Tien, Y.-F. Lee, S.-M. Li, Y.-S. Wang, J.-Y. Wang, C.-C. M. Ma, C.-C. Hu, J. Mater. Chem. 2011, 21, 2374.

[18] J. J. Yoo, K. Balakrishnan, J. Huang, V. Meunier, B. G. Sumpter, A. Srivastava, M. Conway, A. L. M. Reddy, J. Yu, R. Vajtai, P. M. Ajayan, Nano lett. 2011, 11, 1423.

[19] C. D. Zangmeister, Chem. Mater. 2010, 22, 5625.

[20] H. C. Huang, C. W. Huang, C. T. Hsieh, P. L. Kuo, J. M. Ting, H. Teng, J. Phys. Chem. C 2011, 115, 20689.

[21] W. Gao, L. B. Alemany, L. Ci, P. M. Ajayan, Nat. chem. 2009, 1, 403.

[22] B. Xu, S. Yue, Z. Sui, X. Zhang, S. Hou, G. Cao, Y. Yang, Energy Environ. Sci. 2011, 42826. 
[23] X. Fan, W. Peng, Y. Li, X. Li, S. Wang, G. Zhang, F. Zhang, Adv. Mater. 2008, 20, 4490.

[24] O. C. Compton, B. Jain, D. a Dikin, A. Abouimrane, K. Amine, S. T. Nguyen, ACS nano 2011, 5, 4380.

[25] Y. Gogotsi, P. Simon, Science 2011, 334, 917.

[26] H. J. In, S. Kumar, Y. Shao-Horn, G. Barbastathis, Appl. Phys. Lett. 2006, 88, 083104 .

[27] Z. Weng, Y. Su, F. Li, J. Du, Adv. Energy Mater. 2011, 1, 917.

[28] G. Wang, L. Zhang, J. Zhang, Chem. Soc. Rev. 2011, 41, 797.

[29] P. L. Taberna, P. Simon, J. F. Fauvarque, J. Electrochem. Soc. 2003, 150, A292.

[30] V. Presser, L. Zhang, J. J. Niu, J. McDonough, C. Perez, H. Fong, Y. Gogotsi, Adv. Energy Mater. 2011, 1, 423.

[31] J. A. Paradiso, IEEE Pervasive Comput. 2005, 4, 18. 


\section{SUMMARY AND FUTURE WORK}

\subsection{Summary}

This dissertation presents design, fabrication, and evaluation of novel on-chip micro-supercapacitors. The C-MEMS technique was used to fabricate 3D carbon microelectrodes arrays. It was demonstrated that these carbon structure can be directly used for micro-supercapacitors application. The as-fabricated electrodes showed relatively low specific capacitance and energy density. However, After activation of the electrode by a an electrochemical activation technique the specific capacitance was increases up to three order of magnitude compare to the as-fabricated electrodes. This technique was used to fabricate full micro-supercapacitor cells which showed specific geometric capacitance of $\sim 75 \mathrm{mFcm}^{-2}$ was achieved after electrochemical activation for $30 \mathrm{~min}$. For this activation

duration the volumetric capacitance was $\sim 48 \mathrm{Fcm}^{-3}$. These results indicate that the CMEMS technique is a very promising method for the fabrication of electrochemical micro-capacitors

In another part of this dissertation, we examined application of 3D C-MEMS structure as high effective surface area current collector for other capacitive materials. It was observed that the electrochemical properties of C-MEMS micro-supercapacitors can be improved by deposition a conformal film of Ppy on the 3D electrodes. CV and galvanostatic charge/discharge methods were used to evaluate the performance of single micro electrodes and symmetric micro-supercapacitors. Single electrodes with a PPy polymerization time of 15 min can deliver footprint normalized specific capacitance of about $162.07 \mathrm{mFcm}^{-2}$ and specific power of about $1.62 \mathrm{mWcm}^{-2}$ at a $20 \mathrm{mVs}^{-1}$ scan rate. The symmetric micro-supercapacitor delivered specific capacitance of about 78.35 
$\mathrm{mFcm}^{-2}$ at $20 \mathrm{mVs}^{-1}$ scan rate. The increased performance of micro-supercapacitors has enabled them to compete with micro-batteries in a number of applications.

In another approach to develop high power density micro-supercapacitors, we examined application of graphene and its composites with CNT as electrodes for microsupercapacitors. The initial studies were performed on thin films of graphene fabricated through ESD technique. The electrodes show excellent high rate handling ability as it was shown by various electrochemical testing techniques. Electrodes with $1 \mu \mathrm{m}$ thickness showed a much better rate handling ability compared to electrodes with $6 \mu \mathrm{m}$ thickness, highlighting the effect of electrode thickness on the power performance of supercapacitors. At the low discharge rate of $1 \mathrm{Ag}^{-1}$ the specific capacitance of the GNP films with 1 and $6 \mu \mathrm{m}$ thicknesses are about 55 and $53 \mathrm{Fg}^{-1}$, respectively. EIS experiments indicate excellent frequency response of the electrodes and the low RC time constants of 36 and $59 \mathrm{~ms}$ was recorded for electrodes with 1 and $6 \mu \mathrm{m}$ thickness, respectively. Additionally, the demonstrated fabrication process (ESD) is a low-cost technique that could potentially be employed to fabricate thin film electrodes of various kinds of graphene and its composites.

To improve electrochemical properties of graphene based electrodes, microsupercapacitors based single layer rGO and it composites with CNT. The study started with investigation of ESD deposition of GO. We demonstrated that GO sheets in the precursor solution were readily reduced to rGO during the low temperature deposition, eliminating the need for further thermal or chemical reduction of GO. Furthermore, a novel technique by combining photolithography lift-off and ESD deposition was used to fabricated micro-patterned electrodes of GO. For this rGO micro-supercapacitors, the 
diffusion of electrolyte ions between the rGO sheets resulted in electro-activation of the microelectrodes that increased the average $\mathrm{CV}$ current by more than 7 times during the first 200 cycles. We further demonstrated that the addition of CNTs as nano spacers between rGO sheets could minimize their restacking. The electrochemical performance tests indicated that while rGO microdevices had reasonable specific capacitance and power handling ability, the rGO-CNT micro-supercapacitor exhibited exceptional performance. The best results were achieved when a composition of $90 \%$ GO and $10 \%$ CNT was used in the deposition solution. The stack capacitance of rGO-CNT-9-1 microsupercapacitors was about $5.0 \mathrm{Fcm}^{-3}$ at a $1 \mathrm{Vs}^{-1} \mathrm{CV}$ scan rate, which dropped only by $40 \%$ at a very high scan rate of $50 \mathrm{Vs}^{-1}$. The excellent power response of these microsupercapacitors was revealed by EIS experiments when an RC time constant of only 4.8 ms was measured at a $-45^{\circ}$ phase angle, which is lower than any other reported microsupercapacitors. Increasing the amount of CNTs to $20 \%$ slightly improved the power response and rate handling ability of the micro-supercapacitors, but had a negative impact on the specific capacitance. The developed micro-supercapacitors promise high energy micron-scale energy storage units that are able to provide enough energy and satisfy the peak power required for a number of applications. They can also potentially replace low energy electrolytic capacitors in miniaturized electronic devices. It is anticipated that further improvement in the performance of micro-supercapacitors can be achieved through the optimization of ESD conditions, microelectrode design, and the composition of the electrode materials. 


\subsection{Future Work}

This dissertation has introduced various ideas to fabricate micro-supercapacitors with improved electrochemical performance. The proposed strategies suggest new possibilities for development of micro-supercapacitor and their future applications.

In case of micro-supercapacitors based on C-MEMS structure, the proposed activation method still needs to be optimized to achieve improved performance. The study of porous structure of electrochemically activated sample would result in a better understanding of activation mechanism. In the present dissertation, the application of $\mathrm{C}$ MEMS structures as current collectors for other electro-active materials was demonstrate by using Ppy as the capacitive material. This type of micro-supercapacitor can be still explored by using other high energy conduction polymer electrodes such as Polyaniline (PANI) or metal oxide electrodes such as Manganese Oxide $\left(\mathrm{MnO}_{2}\right)$ or composites of these materials with carbon nanomaterials such as CNT and graphene.

One of the major achievements of this dissertation is the development of microsupercapacitors based on patterned rGO. The performance of these type of micro-devices can be improved by optimizing the deposition and reduction processes. The future research should also be focused on integrating graphene and graphene/CNT electrodes on 3D structures to achieve higher energy densities for these micro-supercapacitors. 


\section{MAJID BEIDAGHI}

$1999-2003$

B.Sc., Materials Science Engineering

Isfahan University of Technology

Isfahan, Iran

1978-1979

M.Sc., Materials Science and Engineering

K. N. Toosi University of Technology

Tehran, Iran

2008-2012

Doctoral Candidate $(\mathrm{PhD})$

Florida International University

Miami, Fl, USA

\section{PUBLICATIONS AND PATENTS AND SELECTED PRESENTATIONS}

Majid Beidaghi and Chunlei Wang, "Micro-supercapacitors Based on Interdigital Electrodes of Reduced Graphene Oxide and Carbon Nanotube Composites with Ultrahigh Power Handling Performance" Advance Functional Materials (2012) DOI 10.1002/adfm.201201292

Majid Beidaghi, Zhifeng Wang, Lin Gu, Chunlei Wang, "Ultra-high Power Handling Capability of Binder-free Thin Film Graphene Supercapacitor Electrodes", Journal of Solid State Electrochemistry (2012) DOI 10.1007/s10008-012-1777-5.

Varun Penmatsa, Taekwon Kim, Majid Beidaghi, Hiroshi Kawarada, Zhifeng Wang, Lin Gu, Chunlei Wang “Three-Dimensional Graphene Nanosheets Encrusted Carbon Micropillar Arrays for Electrochemical Sensing”, Nanoscale (2012), DOI:

10.1039/C2NR30161J.

Majid Beidaghi, Chunlei Wang, "Micro-supercapacitors based on three dimensional interdigital polypyrrole/C-MEMS electrodes”, Electrochimica Acta, 56 (2011) 9508-9514

Majid Beidaghi, Wei Chen, Chunlei Wang, "Electrochemically Activated 3D C-MEMS Based Supercapacitors”, J. Power Sources 196 (2011) 2403-2409.

Wei Chen, Majid Beidaghi, Varun Penmatsa, Latha Kumari, Wenzhi Li, and Chunlei Wang, "Integration of Carbon Nanotubes to C-MEMS for On-chip Supercapacitors", IEEE Transactions On Nanotechnology 9 (2010) 734-740. 
Majid Beidaghi, Wei Chen, Chunlei Wang, "Electrochemically Activated C-MEMS Electrodes for On-chip Supercapacitor" US Patent Appl. No. 61/472,591 (filing date: $04 / 06 / 2011)$

Majid Beidaghi, Chunlei Wang," Recent advances in design and fabrication of on-chip micro-supercapacitors" Proc. SPIE 8377, 837708 (2012).

Majid Beidaghi, Chunlei Wang, "Design, fabrication, and evaluation of on-chip microsupercapacitors”, Proc. SPIE 8035, 80350J (2011).

Majid Beidaghi, Chunlei Wang, "On-chip micro-power: Three dimensional structures for micro-batteries and micro-supercapacitors", Proc. SPIE 7679, 76791G (2010).

Majid Beidaghi, Chunlei Wang, "Design, fabrication, and evaluation of on-chip microsupercapacitors", Proceeding of SPIE Symposium on Defense, Security, and Sensing, April 25-29, 2011, Orlando,FL

Majid Beidaghi, Chunlei Wang, "On-chip micro-power: Three dimensional structures for micro-batteries and micro-supercapacitors", Proceeding of SPIE Symposium on Defense, Security, and Sensing, April 5-9, 2010, Orlando,FL

Majid Beidaghi, Chunlei Wang, "Micro-Supercapacitors of Hybrid Graphene/CNT Electrodes." (oral presentation), 2011 MRS Fall meeting, November 28 - December 2, 2011, Boston, MA

Majid Beidaghi, Chunlei Wang, "Hybrid Graphene-Carbon Nanotube Films as Supercapacitor Electrodes", (oral presentation), 220th ECS meeting, October 9-14, 2011, Boston, MA.

Majid Beidaghi, Chunlei Wang, "Electrochemical performance of manganese oxide/graphene composite electrodes prepared by electrostatic spray deposition", (oral presentation), 219th ECS meeting, May 1-6, 2011, Montreal, Canada.

Majid Beidaghi, Chunlei Wang, "Binder-free Porous Network of Graphene/Manganese Oxide Composites as Electrodes for Electrochemical Capacitors" (oral presentation), 2011 MRS Spring meeting, Apri 25-29, 2011, San Francisco, CA.

Majid Beidaghi, Chunlei Wang, "C-MEMS/Manganese Oxide Composites as Electrodes for Microsupercapacitors" (oral presentation), 2011 MRS Spring meeting, April 25-29, 2011, San Francisco, CA.

Majid Beidaghi, Chunlei Wang, "C-MEMS Structures as Three Dimensional Current Collectors for Micro supercapacitors" (oral presentation), 2010 MRS Fall meeting, November 29 - December 3, 2010 Boston, MA. 
Majid Beidaghi, Chunlei Wang, " Preparation and electrochemical characterization of polypyrrole-based three dimensional micro-supercapacitors" (oral presentation), 218th ECS meeting, October 10-15, 2010, Las Vegas, NV.

Majid Beidaghi, Wei Chen, Chunlei Wang "Electrochemically Activated C-MEMS Structures as Microelectrodes for On-Chip Supercapacitors" (oral presentation), 2010 MRS Spring meeting, April 6-8, 2010, San Francisco, CA.

Majid Beidaghi, Wei Chen, Chunlei Wang, "Three Dimensional Microsupercapacitors: As-Pyrolyzed and Porous Carbon Structures" (poster), 2009 MRS fall meeting, Nov 30Dec 4, 2009, Boston, MA.

Majid Beidaghi, Wei Chen, C. Wang, "3D Microsupercapacitor Based on C-MEMS with Improved Electrode Design” (oral presentation), Nano-DDS 2009, Sept 28-Oct 2, 2009, Ft Lauderdale, FL.

Majid Beidaghi, Varun Penmatsa, Wei Chen, Chunlei Wang, "On-Chip Supercapacitors Based on Porous Carbon Electrodes", (poster) 215th ECS Meeting, May 24-29, 2009, San Francisco, CA

Majid Beidaghi, Varun Penmatsa, Wei Chen, Chunlei Wang, "Characterization of Porous Electrodes for 3D Microsupupercapacitors", (oral presentation) 25th SBEC, May 15-17, 2009, Miami, FL.

Majid Beidaghi, Varun Penmatsa, Yan Yu, Chunlei Wang, "3D Microsupercapacitor Based on C-MEMS with Improved Electrode Design” (poster) 2008 MRS Fall Meeting, Dec 1-5, 2008, Boston, MA. 